\title{
Discrete Breathers
}

\author{
S. Flach \\ Max-Planck-Institut für Physik komplexer Systeme, Bayreuther Str.40 H.16, D-01187 Dresden, Germany \\ C. R. Willis \\ Department of Physics, Boston University, Boston, MA 02215, USA
}

(February 4, 2008)

Nonlinear classical Hamiltonian lattices exhibit generic solutions in the form of discrete breathers. These solutions are time-periodic and (typically exponentially) localized in space. The lattices exhibit discrete translational symmetry. Discrete breathers are not confined to certain lattice dimensions. Necessary ingredients for their occurence are the existence of upper bounds on the phonon spectrum (of small fluctuations around the groundstate) of the system as well as the nonlinearity in the differential equations. We will present existence proofs, formulate necessary existence conditions, and discuss structural stability of discrete breathers. The following results will be also discussed: the creation of breathers through tangent bifurcation of band edge plane waves; dynamical stability; details of the spatial decay; numerical methods of obtaining breathers; interaction of breathers with phonons and electrons; movability; influence of the lattice dimension on discrete breather properties; quantum lattices - quantum breathers.

Finally we will formulate a new conceptual aproach capable of predicting whether discrete breather exist for a given system or not, without actually solving for the breather. We discuss potential applications in lattice dynamics of solids (especially molecular crystals), selective bond excitations in large molecules, dynamical properties of coupled arrays of Josephson junctions, and localization of electromagnetic waves in photonic crystals with nonlinear response.

pacs: 03.20.+i , 63.20.Pw , 63.20.Ry

keywords: lattices, breathers, localization

To be published in Physics Reports 


\section{Contents}

I Introduction 6

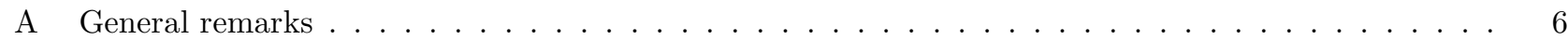

B Nonlinearity and Discrete Breathers . . . . . . . . . . . . . . . . . . . . . . . . . 6

C Some Properties of breathers in Hamiltonian Field Equations . . . . . . . . . . . . . . . . . . . . . . . 7

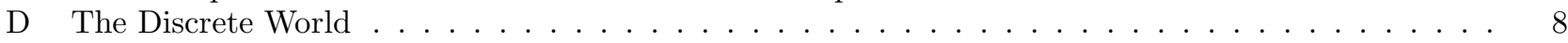

II $\quad$ First approximate results 8

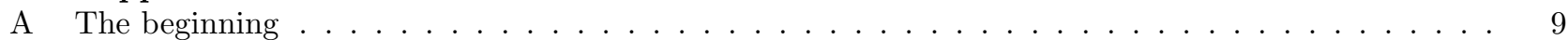

B Multiple scale expansions . . . . . . . . . . . . . . . . . . . . . . . . . . . . . . 9

C Rotating wave approximation . . . . . . . . . . . . . . . . . . . . . 10

D $\quad$ Numerical evidence $\ldots \ldots \ldots \ldots \ldots \ldots$

III The local ansatz 12

A A numerical experiment . . . . . . . . . . . . . . . . . . . . . . . . . . 12

B The internal dynamics . . . . . . . . . . . . . . . . . . . . . . . . . . 13

C The reduced problem . . . . . . . . . . . . . . . . . . . . . . . 13

D A correspondence conjecture . . . . . . . . . . . . . . . . . . . . . . . . . . . 13

E Two-dimensional lattic . . . . . . . . . . . . . . . . . . . . . . . . . . . . . . . . 14

F Wavelet analysis . . . . . . . . . . . . . . . . . . . . . . . . . . . 15

IV Localization properties of discrete breathers 15

A The ansatz for a breather solution . . . . . . . . . . . . . . . . . . . . . . . . . . 15

B Existence conditions for time-periodic discrete breathers . . . . . . . . . . . . . . . . . . . 16

C Quasiperiodic discrete breathers . . . . . . . . . . . . . . . . . . . . . 17

D The spatial decay of DBs . . . . . . . . . . . . . . . . . . . . . . . . . . . . 18

E Nonlinear corrections . . . . . . . . . . . . . . . . . . . . . . . . 18

F The decay in $k$-space . . . . . . . . . . . . . . . . . . . . . . . . . . . . . . . . . 19

G The case $v_{2}=0$. . . . . . . . . . . . . . . . . . . . . . . . . . . . . . 19

$\mathrm{H}$ Systems without a linear spectrum . . . . . . . . . . . . . . . . . . . . . . . . . . 19

Higher lattice dimensions . . . . . . . . . . . . . . . . . . . . . . . . . . . . . . 20

\begin{tabular}{|lll}
\hline Existence proofs & 20
\end{tabular}

A The antiintegrability approach . . . . . . . . . . . . . . . . . . . . . . 20

B The homoclinic orbit approach . . . . . . . . . . . . . . . . . . . . . . . . . . 21

\begin{tabular}{|lll}
\hline VI & Numerical methods obtaining discrete breathers & 22
\end{tabular}

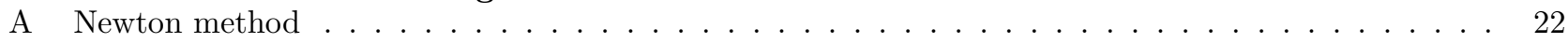

1 Phase space variables . . . . . . . . . . . . . . . . . . . . . . . . . . 23

2 Fourier coefficients. . . . . . . . . . . . . . . . . . . . . . . . . . 23

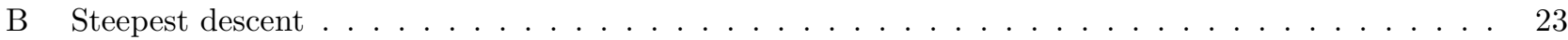

1 Phase space variables . . . . . . . . . . . . . . . . . . . . . . . . . . . . . 24

2 Fourier coefficients. . . . . . . . . . . . . . . . . . . . . . . . . 24

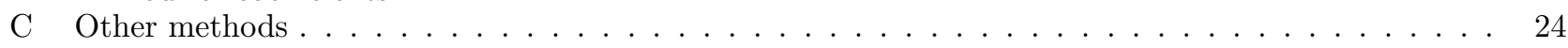

VII $\quad$ Structural stability 25

VIII Dynamical stability 25

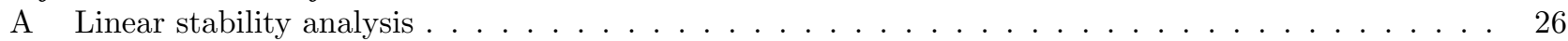

B Going beyond linearization . . . . . . . . . . . . . . . . . . . . . . 27

IX Movability

A Numerical findings . . . . . . . . . . . . . . . . . . . . . . . . . . . . 28

B What can we learn from moving lattice kinks? . . . . . . . . . . . . . . . . . . . . . . . . . . . 29

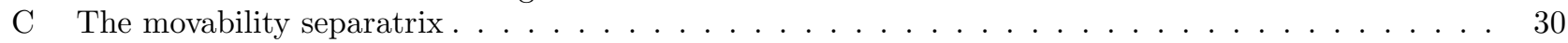

D Breather-breather collisions . . . . . . . . . . . . . . . . . . . . . . . 32

E Do moving breathers exist? . . . . . . . . . . . . . . . . . . . . . . . . . . . 32 


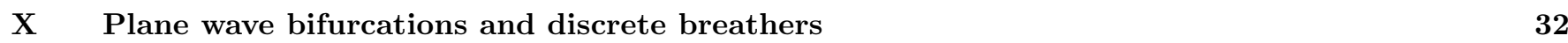

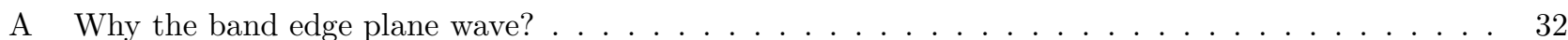

B Tangent bifurcations of band edge plane waves . . . . . . . . . . . . . . . . . . . . 33

1 Tangent bifurcation of orbit $1 \ldots \ldots \ldots \ldots \ldots \ldots \ldots \ldots$

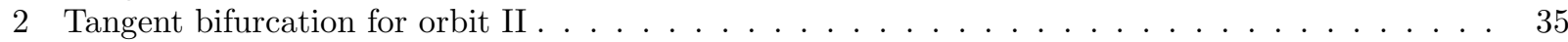

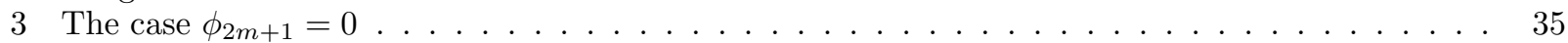

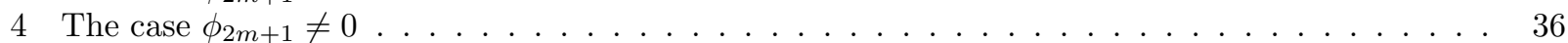

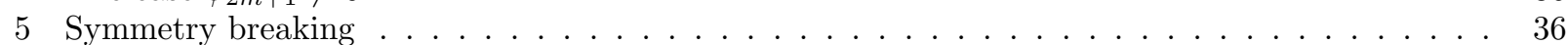

C Let us predict! . . . . . . . . . . . . . . . . . . . . . . . . . . . 36

\begin{tabular}{|ll}
\hline XI $\quad$ Lattice dimension effects & 37 \\
\hline
\end{tabular}

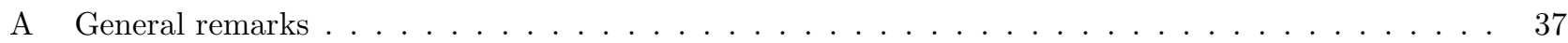

B Energy thresholds for discrete breathers . . . . . . . . . . . . . . . . . . . . . . . . . . 37

\begin{tabular}{llr}
\hline XII & Discrete symmetries & 38
\end{tabular}

\begin{tabular}{|rr}
\hline XIII A conceptual approach & 39
\end{tabular}

\begin{tabular}{|ll}
\hline XIV & Plane wave scattering by discrete breathers \\
\hline
\end{tabular}

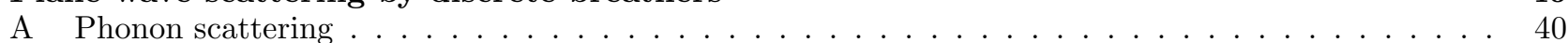

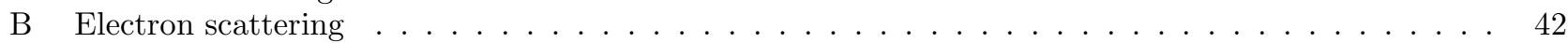

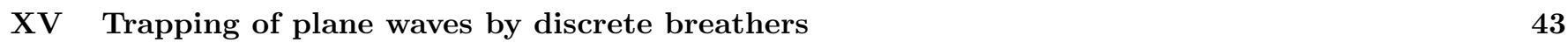

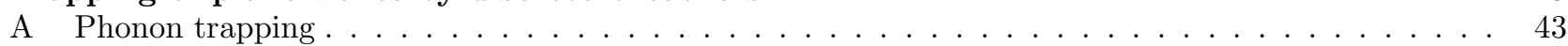

B Electron trapping . . . . . . . . . . . . . . . . . . . . . . . . . . . . . . 44

1 Model structure . . . . . . . . . . . . . . . . . . . . . . . . . . . 44

2 Holstein model and nonlinear generalizations . . . . . . . . . . . . . . . . . . . . . 45

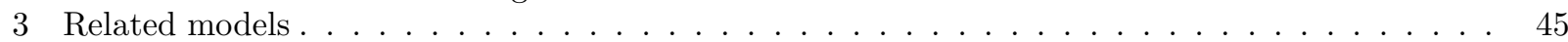

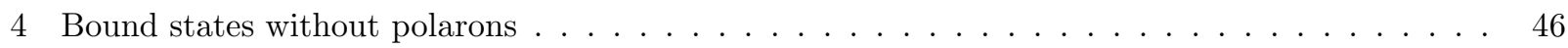

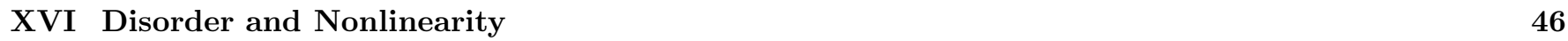

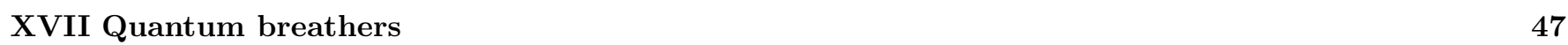

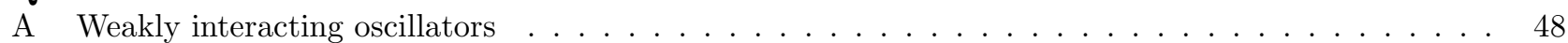

B Systems with boson number conservation . . . . . . . . . . . . . . . . . . . . . . . . . 49

1 Two bosons $\ldots \ldots \ldots \ldots \ldots \ldots \ldots \ldots \ldots$

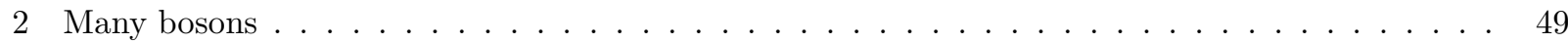

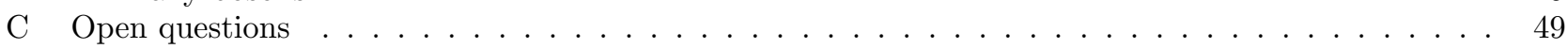

\begin{tabular}{|rr}
\hline XVIIIPossible experimental realizations & 50
\end{tabular}

\begin{tabular}{ll}
\hline XIX Conclusions & 51 \\
\hline
\end{tabular}

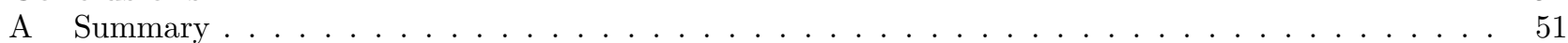

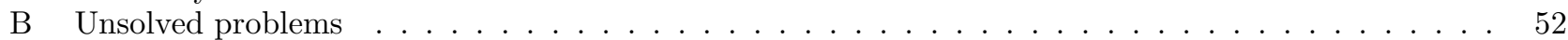

C Some closing words $\ldots \ldots \ldots \ldots \ldots \ldots \ldots \ldots \ldots \ldots \ldots$

\begin{tabular}{ll}
\hline$X X$ & What's new? \\
\hline
\end{tabular} 


\section{LIST OF FREQUENTLY USED ABBREVATIONS}

$\begin{array}{ll}\text { ODE } & \text { ordinary differential equation } \\ \text { PDE } & \text { partial differential equation } \\ \text { DB } & \text { discrete breather } \\ \text { NLS } & \text { nonlinear Schrödinger equation } \\ \text { DNLS } & \text { discrete nonlinear Schrödinger equation } \\ \text { AL } & \text { Ablowitz-Ladik (lattice) } \\ \text { RWA } & \text { rotating wave approximation } \\ \text { FPU } & \text { Fermi-Pasta-Ulam (model) } \\ \text { KG } & \text { Klein-Gordon (model) } \\ \text { SG } & \text { sine-Gordon (model) } \\ \text { PM } & \text { Poincare map } \\ \text { PBC } & \text { periodic boundary conditions }\end{array}$


Dedicated to Peter Fulde on the occasion of his 60th birthday. 


\section{INTRODUCTION}

Discreteness is at the very heart of almost any many-particle system, if the processes under discussion involve length scales of the order of (or not too large compared to) the interparticle distances. Condensed Matter physics is a particular well-known area of research where discreteness plays an important role, and the same could also be said of topics in chemistry or biology. Discreteness is here typically meant with respect to space (particles versus fields). Consequently one deals with Hamiltonians which are discrete in space but define a continuous phase space flow with respect to time.

In this review we will discuss time-periodic localized excitations in discrete systems. The localization is due to the discreteness combined with the nonlinearity of the system, as opposed to the well known disorder induced localization. The reader is encouraged to check the subsection 'Summary' of the conclusions to this report for a full list of the results discussed. Some of the results discussed here can be found in [162], [79], [13].

\section{A. General remarks}

Typically the dynamics of a many-particle system can be regarded as a discretization of certain continuous systems, that is the classical coupled ordinary differential equations (ODE) of the many-particle system can be regarded as discretized versions of partial differential equations (PDE). The replacement of the spatial derivatives by spatial differences in the equations of motion implies the reduction of the symmetry of the Hamiltonian. In general lowering the symmetry means enriching the class of solutions because less restrictions are imposed. Of course one also looses solutions when lowering the symmetry - namely the ones which were generated by the higher symmetry. Let us discuss two simple examples of linear equations (PDE or ODE). Assuming that the PDE has a continuous translational symmetry, we can expect a linear spectrum (i.e. a dispersion relation) $\omega(q)$ which is unbounded. The discrete version related to the PDE will yield a linear spectrum which is i) bounded and ii) might have additional gaps depending on the discretization procedure. The finite upper (or lower) bound of the linear spectrum is intimately connected with the interparticle distance (or the discretization step of the space continuum). Replacing e.g. the wave equation $U_{, t t}-U_{, x x}=0$ by $U(l)_{, t t}-(U(l+1)+U(l-1)-2 U(l))=0$ with $l=0, \pm 1, \pm 2, \ldots$ means replacing the linear spectrum $\omega^{2}(q)=q^{2}$ by $\omega^{2}=2(1-\cos (q))$. Let us now add a translational symmetry breaking inhomogeneity to the system in the form $(1+\epsilon \delta(x)) U_{, t t}-U_{, x x}=0$ for the continuum and $\left(1+\epsilon \delta_{l, 0}\right) U(l), t t-(U(l+1)+U(l-1)-2 U(l))=0$ for the lattice. For $\epsilon<0$ it is well-known that a spatially localized and time-periodic solution (eigenmode) exists for the lattice version. This happens because the linear spectrum has a finite bound, and eigenmodes with frequencies outside the linear spectrum can not be spatially extended. However the corresponding PDE does not posess a bound state, simply because the linear spectrum occupies the whole real axis. Indeed, substituting $U(x, t)=u(x) \mathrm{e}^{i \omega t}$ we obtain $-u_{, x x}+\omega^{2}(-1+\epsilon \delta(x)) u(x)=0$ which is the time-independent Schroedinger equation of a particle in the potential $V(x)=\omega^{2}(-1+\epsilon \delta(x))$. Since the corresponding eigenenergy is zero, obviously no bound state exists - rather an extended state is found. The pattern of thoughts which leads to the last result without performing all calculations is: i) if we discretize a PDE then the linear spectrum will be bounded and might even lead to additional gaps in the PDE linear spectrum (think of electronic band structures in solids); ii) thus we can by proper modifications of the discretized equations produce spatially localized but time-periodic states, whose frequencies are located in the new gaps opened due to discreteness.

\section{B. Nonlinearity and Discrete Breathers}

As long as we stick to linear equations, we have to break the discrete translational symmetry of the discretized equations in order to obtain spatially localized modes, i.e. we have to add 'impurities'. In this review we will show that one can retain the discrete symmetry and still obtain localized excitations - by adding nonlinear terms to the equations. Certainly nonlinear terms are present in any application of many-particle dynamics. The thought pattern capable of predicting the existence of localized excitations will be much more elaborate than for the linear case. We will see that due to the nonlinearities higher harmonics of the excitation's frequency have to be considered too (i.e. their positions with respect to the linear spectrum). Consequently nonlinearity is crucial with respect to the difference between continuum and discreteness. Indeed, the above demonstrated linear examples can not change the fact that one can add terms to the PDE which cause the opening of gaps (and still preserve the continuous symmetry). Then nothing hinders us from adding symmetry-perturbing terms which are capable of producing localized or bound states. However in the case of a nonlinear PDE the fact that the linear spectrum is still unbounded typically forbids the existence of bound states (because the mentioned higher harmonics of the bound state's frequency will always resonate 
with the linear spectrum). Here is the big advantage of a discrete system, because the finite upper bound of the linear spectrum still allows for frequencies whose entire higher harmonics might lie outside the linear spectrum.

Thus we are going to investigate solutions of Hamiltonian lattice equations (e.g. describing the classical dynamics of a crystal lattice) which are periodic in time and localized in space. Different names of these objects have appeared in the literature - Intrinsic Localized Modes (ILM), Self-localized Anharmonic Modes (SLAM), Nonlinear Localized Excitations (NLE), Discrete Breathers (DB), etc. The first three entries of this exotic list are perhaps an attempt to separate the appearance of these objects from some well-known but only weakly related solutions of PDEs - namely breathers. The fourth entry of the list tries to create the difference by the attribute 'discrete'. We choose the entry 'discrete breather' (although we used NLE before). The main reason is that the objects are indeed breathers living on a lattice, so it is sort of confusing to create new names. Perhaps 'lattice breathers' would be better? There is still some dynamics in that issue, so we will have to wait and see which entry will be dominant.

\section{Some Properties of breathers in Hamiltonian Field Equations}

Breather solutions are well-known from the study of the sine-Gordon (sG) PDE. The sG equation for a real-valued field $\Psi(x, t)$ is a particular example out of the class of nonlinear Klein-Gordon (KG) equations

$$
\Psi_{, t t}=C \Psi_{, x x}-F(\Psi)
$$

with the choice $F_{s G}(z)=\sin z$. The breather solution is given by

$$
\Psi_{b}(x, t)=4 \tan ^{-1}\left[\frac{m}{\omega} \frac{\sin (\omega t)}{\operatorname{ch}(m x)}\right], \omega=\sqrt{1-m^{2}} .
$$

It represents a field which is periodically oscillating in time and decays exponentially in space as the distance from the center $x=0$ is increased (see Fig.1). As shown stationary breather solutions form one-parameter families of solutions.

Most probably these breather solutions are nongeneric. Birnir [24], 23] and Denzler [54], [55] showed that sG breathers are isolated, i.e. the solutions survive only under a finite number of perturbations $\delta(z)$ of $F_{s G}(z) \rightarrow$ $F_{s G}(z)+\delta(z)$. Years of searching for breathers in $\phi^{4}$ systems $\left(F(z)=-z+z^{3}\right)$ were terminated by the nonexistence proof of breathers by Segur and Kruskal [160]. Related results have been derived by Kichenassamy [116]. Geicke [97] has calculated the decay rate of $\phi^{4}$-'breathers'. So it appears that breathers are nongeneric (structurally unstable) and thus rare objects in PDEs. The reason for that lies in the fact that a decomposition of (1.2) into a Fourier series with respect to time yields higher harmonics with frequencies $k \omega$ ( $k$ is the Fourier number). These frequencies resonate with the linear spectrum of (1.1) $\omega_{q}=\sqrt{C q^{2}+F^{\prime}(z=0)}$ ( $q$ - wave number), if $k$ is larger than a given number which depends on the choice of $\omega$. Consequently the corresponding separatrix manifolds associated with (1.1) are of finite dimension. The intersection of finite-dimensional manifolds in an infinite-dimensional phase space is comparable to a miracle - translated this implies an infinite number of symmetries. Indeed the sG equation is integrable! Together with the infinity of the dimension of the corresponding phase space the structural instability of $\mathrm{sG}$ breathers follows immediately [66]. It is interesting to note that those arguments are obtained by linearising the equation (1.1) and then analyzing the ODEs for the Fourier coefficient functions. However a test of solution (1.2) shows that the linearization is not adequate - simply because (1.2) has nonzero higher Fourier components (with respect to time) and those components would not spatially decay in the linearized version of (1.1). We will discuss similar effects in the lattice case.

Boyd [34] introduced a very interesting concept of the 'nanopteron' (still another exotic name). The nanopteron concept is the following: assume that the PDE does not allow for a breather. Then it can still allow for a solution which is locally close to a breather, but which does not decay to zero in space - rather it decays to some nonzero but small (compared with the center) amplitude. The essential difference between the breather and the nanopteron is that breathers are solutions of finite energies, whereas nanopterons are solutions of infinite energies. The nanopteron construction essentially uses the fact that there can be solutions periodic in time with frequencies outside the linear spectrum. Because higher harmonics resonate with the linear spectrum, the solutions do not decay in space to zero, but nevertheless keep their inhomogeneous spatial properties.

It is worthwhile mentioning another field equation which supports breather solutions - the Nonlinear Schrödinger Equation (NLS) in $1+1$ dimensions:

$$
-\mathrm{i} \Psi_{, t}=\Psi_{, x x}+2|\Psi|^{2} \Psi
$$

This equation is also integrable (!) (note that the field is complex-valued) and posesses soliton solutions [58]. One of them is nothing but a breather solution. The breather solution is obtained by substituting $\Psi(x, t)=\phi(x) \mathrm{e}^{\mathrm{i} \omega t}$. The time-independent field $\phi$ is assumed to be real valued and satisfies the equation 


$$
\phi_{, x x}=\omega \phi-2 \phi^{3}
$$

For $\omega>0$ this equation allows for homoclinic orbits defined by $\phi(0)=\sqrt{\omega}, \phi_{x}(0)=0$. These homoclinic orbits yield breather solutions of the original NLS equation (1.3). It is interesting to note that in contrast to the sG case perturbations of the NLS equation sometimes do not destroy the breather solutions. However they will not destroy the breather solution typically only if the perturbations preserve the conservation law $B=\int|\Psi|^{2} \mathrm{~d} x$. This is intimately connected to the nongeneric structure of the NLS equation itself - namely that the nonlinearity appears only in the amplitudes, but not in the phases (of the complex field $\Psi$ ).

We have found only one example of a PDE in $2+1$ dimensions which allows for numerically obtained breather-like objects [6]. Also we want to mention the efforts to obtain breather solutions for Einstein-Maxwell equations of the electro-vacuum, and for equations describing one-dimensional optical media with Kerr nonlinearities capable of selfinduced transparency [182], [67]. Altogether the efforts of finding breathers in PDEs have been discouraging. It is hard to believe that isolated, nongeneric and structurally unstable objects are capable of describing phenomena in nature.

\section{The Discrete World}

Although there has been interest in the relationship between the continuum and discreteness for a considerable amount of time many of the results present here are quite new.

One possible way of testing the difference between PDEs and their discretized versions with respect to breathers would be to find a lattice model with discrete breathers and then to test whether they are structurally stable or not. Indeed we can write down one such lattice model - the Ablowitz-Ladik (AL) lattice [1]

$$
i \Psi(l)_{, t}+C(\Psi(l+1)+\Psi(l-1)-2 \Psi(l))+(\Psi(l+1)+\Psi(l-1))|\Psi(l)|^{2}=0
$$

The AL lattice is a discretized version of the NLS equation, and the AL lattice is integrable. It allows for breather solutions. The structural stability analysis of AL breathers has not been done yet. Also any structural stability analysis will not yield too much insight into why breathers might exist for many different lattice realizations. Thus we choose a different way of analysis. At the end of this analysis we will obtain a conceptual approach which allows us to predict the existence/nonexistence of breathers for lattices without actually solving for the breather solutions.

\section{FIRST APPROXIMATE RESULTS}

In this section we will introduce the models, discuss multiple scale expansions and rotating wave approximations as well as some numerical studies which have been used in order to describe discrete breathers.

Let us consider a $d$-dimensional hypercubic lattice with $N$ sites. Each site is labeled by a $d$-dimensional vector $l \in Z^{d}$. Assign to each lattice site a vector $X_{l} \in R^{f}$, where $f$ is the number of components and is to be finite. The evolution of the system is given by the set of equations

$$
\dot{X}_{l}=F_{l}\left(\left\{X_{m}\right\}\right), F_{l}(\{0\})=0
$$

It will be important for the discussion to agree upon a general form of the expansion of $F$ in the variables $X$, which is usually a Taylor expansion:

$$
F \sim \sum_{\mu=1}^{\infty} f_{\mu} X^{\mu}
$$

In fact the expansion coefficients in $(2.2)$ are tensors reflecting the true topology of the interaction incorporated in the general form (2.1). For finite number of sites $N$ we impose periodic boundary conditions. Then the discrete translational invariance of the system implies that any solution of (2.1) generates new solutions under translation of all $l$ by the same vector $L \in Z^{d}: l \rightarrow l+L$. According to (2.1) $X_{l}=0$ is a solution for all times, hereafter coined groundstate solution (GS). Finally the evolution defined by (2.1) is required to have an integral of motion (energy) $E\left(\left\{X_{l}\right\}\right) \geq 0, E(\{0\})=0$ with $\dot{E}=0$. In the following we will briefly discuss various perturbation theories applied to specifications of (2.1). 


\section{A. The beginning}

In preparing this report we came across a remarkable paper by Kosevich and Kovalev [131] (hereafter referred to as $\mathrm{KK}$ ) which seems to be the first direct attempt to solve for discrete breathers. Furthermore the authors of this early work addressed a whole set of questions connected with the existence of discrete breathers, which will be discussed in detail in coming sections - e.g. the connection of the existence of discrete breathers with instabilities of band edge plane waves. One of the models studied is given by equation (1) in [131:

$$
\ddot{u}_{n}+b^{2}\left(2 u_{n}-u_{n+1}-u_{n-1}\right)-f\left(u_{n}\right)=0 \text {. }
$$

This is a discretized version of the Klein-Gordon equation in $1+1$ dimensions (and is a special case of (2.1) with $X_{n}$ being a two-component vector with the components $u_{n}$ and $\left.\dot{u}_{n}\right)$. The function $f(z)$ is set to $f(z)=-z+\alpha z^{2}+\beta z^{3}$. Linearization of (2.3) around the stable static solution $u_{n}(t)=0$ yields the linear spectrum (i.e. dispersion relation) for plane waves $\omega_{q}^{2}=1+2 b^{2}(1-\cos q)$. Note that the linear spectrum has i) a finite upper bound - this is important with respect to higher harmonics of a breather solution, and ii) a lower nonzero bound - this allows for breather frequencies below the linear spectrum (sometimes called gap modes). KK consider solutions of (2.3) with frequencies below and close to the lower band edge of the linear spectrum $\omega=1$. Intuitively those solutions should be of large wavelength, thus KK argue in favor of considering the PDE $u_{, t t}-u_{, x x}-f(u)=0$. Breather solutions are then derived using multiple scale expansions. In lowest order the solution reads (equation (8) in [131])

$$
u(x, t)=\left(\frac{8}{3 \beta}\right)^{1 / 2} \frac{\sqrt{1-\omega^{2}}}{\operatorname{ch} \sqrt{1-\omega^{2}} x} \cos \omega t .
$$

However 13 years later Segur and Kruskal have demonstrated that those asymptotic expansions are not correct [160]. Still the analysis of KK is impressive, especially the idea that also solutions with frequencies larger than and close to the linear spectrum can be considered in a continuum limit after proper substitutions. However the continuum approximation is clearly not appropriate for the discussion of breather solutions, because the breather solution will inevitably have higher harmonics with respect to a Fourier series in time, and those higher harmonics will ultimately resonate with the unbounded linear spectrum of the PDE. However the starting point was (2.3) whose linear spectrum is bounded from above.

\section{B. Multiple scale expansions}

Further progress using multiple time scales was achieved by Remoissenet [150] (see also [127, [27). This semidiscrete multiple-scale approach keeps the discreteness yet still uses the frequency distance from the band edge of the linear spectrum as a small parameter. Typically one looks for solutions in the form

$$
u_{n}=\phi_{n}+\left(\psi_{n} \mathrm{e}^{i t}+c c\right)+\left(\xi_{n} \mathrm{e}^{2 i t}+c c\right)+\ldots
$$

where the functions $\phi_{n}, \psi_{n}, \xi_{n}, \ldots$ are small in amplitude and slowly varying in time. Using $\epsilon=1-\omega$ as a small parameter (where $\omega$ is some frequency of the solutions we are looking for) and assuming $\psi_{n} \sim \sqrt{\epsilon}, \phi_{n} \sim \epsilon, \xi_{n} \sim \epsilon$, $d / d t \sim \epsilon$ one finally arrives at the celebrated discrete nonlinear Schroedinger equation (DNLS) (we do not care about the explicit form of the coefficients, so only the general form of the equation is given):

$$
i \dot{\psi}_{n}+\left(\psi_{n-1}+\psi_{n+1}-2 \psi_{n}\right)+\kappa\left|\psi_{n}\right|^{2} \psi_{n}=0
$$

This is a remarkable equation which has been studied in great detail and has been used to describe many different phenomena (sometimes this equation is called discrete self-trapping equation 65) Here we want only to discuss its connection to discrete breathers. Let us assume $\psi_{n}=A_{n} \mathrm{e}^{i \epsilon t}$ with $A_{n}$ being real-valued and time-independent, then we obtain

$$
-(\epsilon+2) A_{n}+A_{n-1}+A_{n+1}+\kappa A_{n}^{3}=0
$$

\footnotetext{
${ }^{1}$ For a list of publications on the DNLS click on web page: http://www.ma.hw.ac.uk/ chris/dst/.
} 
Equation (2.7) defines a two-dimensional discrete map (knowing two neighbouring amplitudes allows for the computation of the next amplitude). We are looking for solutions of this map which are finite in some region of the chain and decay to zero if we iterate the map to plus or minus infinity. Solutions of that type are homoclinic orbits and arise from the intersection of the invariant manifolds of a given hyperbolic fixed point. Thus we have to check whether the fixed point solution $A_{n}=0$ is a hyperbolic one, and if yes, whether the invariant stable and unstable manifolds intersect. The first question is easy to solve. For that one linearizes (2.7) around $A_{n}=0$, obtains the matrix defining the linear two-dimensional map and calculates its eigenvalues. The eigenvalues are $\lambda=1+\epsilon / 2 \pm \sqrt{\epsilon+(\epsilon / 2)^{2}}$. For $\epsilon>0$ the fixed point $A_{n}=0$ is hyperbolic and thus two invariant one-dimensional manifolds exist, which originate at the fixed point. The second task - showing that these manifolds intersect - is harder to achieve. However reliable numerical methods exist, and Hennig has shown the intersection of the invariant manifolds [103]. Strictly speaking equation (2.7) has to be analysed in the limit of small $\epsilon \ll 1$, due to the multiple scale expansions. In that case the amplitudes will be small too, and the variation on the lattice is slow compared to the lattice spacing, so that one could replace the differences in (2.7) by differentials. That would bring us back to KK's result (2.4). But we will know that the persistence of the manifold intersection in the discrete system allows for an approximate analytical solution of (2.7) by (2.4). The DNLS (2.6) is an interesting model by itself, i.e. without tying it to the multiple-scale expansion. We will come back to it in the later course of this work.

Let us discuss the multiple-scale expansions in general. First of all when stopping at the first nontrivial order of the expansion (2.6) we did not advance much as compared to the more crude replacement of the difference equations by differential ones as done by KK. Indeed the whole problem of higher harmonics of the breather's frequency resonating (or not) with the linear spectrum appears only in higher orders of the expansion. Bang and Peyrard [17] proceeded to higher orders of the expansion and found several complications. The interested reader will find details in [17]. Here we want to mention two principal problems with those expansions. The multiple-scale expansions attempt to make a perturbation approach to the linear problem which is perturbed with nonlinear terms. However the nonlinear terms in fact destroy the integrability of the linear problem. So what the perturbation approach faces is a nonintegrable perturbation of an integrable problem. This has been quite a topic in mathematics of this century [10]. One type of mathematical approach focus on analytical continuation of solutions of the integrable case into the nonintegrable one (e.g. the famous Kolmogorov-Arnold-Moser theorem continues tori, but only for finite dimensional systems [10]). Another type of approach tries to establish new phase space properties of the nonintegrable case, still using the distance to the integrable system as a small parameter. This second type of approach is very delicate, and the attempt to find discrete breathers as described above belongs to the second type - simply because no breathers exist in the linear (integrable) case (indeed, if we choose $\epsilon=0$ which corresponds to the linear limit, the fixed point $A_{n}=0$ in (2.7) is not hyperbolic anymore). However the first type of approach is useful for describing discrete breathers as we will see. It can be used either to analytically continue discrete breather solutions from some trivial integrable limit, or it can be used to analytically continue plane wave solutions of the linear integrable limit and study their stability properties. Since only periodic orbits will be continued, the continuation can be carried out equally for a finite-dimensional as for an infinite-dimensional phase space!

\section{Rotating wave approximation}

Another approximate method frequently used in the literature is the rotating wave approximation (RWA) [177, [163], [167], [174], [169], [190]. This approach is directly searching for discrete breather solutions as solutions harmonic in time. The solution is parametrized using a Fourier series expansion

$$
u_{n}(t)=\sum_{k=-\infty}^{k=+\infty} A_{n k} \mathrm{e}^{i k \omega t} .
$$

Then one inserts the ansatz into the original equations of motion, collects terms with equal harmonics $k \cdot \omega, k=$ $0, \pm 1, \pm 2, \ldots$ and neglects all coefficients in the Fourier representation for $|k| \geq k_{0}$ for some $k_{0}$. Usually one puts $k_{0}=2$. In that case the RWA is very close to the mentioned multiple-scale expansions. Indeed the RWA is nothing but a perturbation theory of weakly nonlinear oscillations for small amplitudes. This can be immediately obtained for system (2.3) with $\alpha=0$ (cf. text below (2.3). Then $A_{n 0}=0$ for all $n$ (in fact $A_{n, 2 m}=0$ due to the additional symmetry of the Hamiltonian being invariant under change of sign of the variables). We find

$$
-\omega^{2} A_{n, 1}+b^{2}\left(2 A_{n, 1}-A_{n+1,1}-A_{n-1,1}\right)+A_{n, 1}-6 \beta A_{n, 1}^{3}=0 \quad .
$$

The similarity to equation (2.7) is evident. However the RWA has been frequently used for quite strong nonlinearities. For instance for the trivial case $b=0$ (independent sites) we solve (2.9) for any site and find $A_{n, 1}=0$ or $A_{n, 1}=\omega / \sqrt{6 \beta}$. 
We can choose any of the two solutions at any lattice site and thus create an infinite number of solutions characterized by a binary sequence. The position of an element of the sequence is the lattice site, and the value of the element encodes either of the two possible solutions for $A_{n, 1}$. Using Aubry's 'antiintegrability' approach 14] it is possible to prove continuation of those solutions into the interacting $b \neq 0$ case. If we would choose e.g. a sequence $(. .00001000 \ldots)$ the continuation into the interacting case would correspond to a solution which is still spatially localized - and could be called a discrete breather. The richness of the possible internal structure of localized solutions is evident due to the arbitrariness in the choice of the coding sequence - e.g. why not take (...000101011000...)?

The reason why the RWA can give quite reasonable estimations even when being far away from the usual perturbation region of amplitudes has been explained in [90] by studying a single nonlinear oscillator. We apply the RWA to the one particle problem

$$
\ddot{Q}=-V^{\prime}(Q) \text {. }
$$

The potential $V(Q)$ should provide bound motion, at least for the cases under consideration. Since this problem is integrable, one can (at least numerically) calculate the period of the periodic solution (2.10) and thus the fundamental frequency. E.g. for the class of potentials

$$
V(Q)=\frac{1}{2 m} Q^{2 m}
$$

the solution for the frequency can be found analytically as a function of the energy $E$ :

$$
\omega=\frac{1}{2} \sqrt{2 \pi}(2 m)^{1-1 / 2 m} \cdot \frac{\Gamma(1 / 2 m+1 / 2)}{\Gamma(1 / 2 m)} \cdot E^{1 / 2-1 / 2 m} .
$$

Here $\Gamma(x)$ is the Gamma-function. Applying RWA with $k_{o}=2$ one derives the approximation

$$
\omega_{1}=2^{1-m} \cdot(2 m)^{1 / 2-1 / 2 m} \cdot \sqrt{\frac{(2 m-1) !}{m !(m-1) !}} \cdot E^{1 / 2-1 / 2 m} .
$$

First we note the remarkable coincidence between the exact result (2.12) and the approximation (2.13) with respect to the energy dependence, which was achieved within the RWA using the exact relation between the amplitude and the energy as it follows from (2.11). Moreover comparing the prefactors for e.g. $m=2$ in (2.11) yields 1.1981 and 1.2247 for the exact result (2.12) and the approximation (2.13), respectively. That means that the simplest RWA in the case of (2.11) already gives an error of less then $2.3 \%$ ! The errors accumulate however for larger $m$. In the limit $m \rightarrow \infty$ the potential (2.11) becomes box-like shaped, and the approximation of the dynamics in this potential by a harmonic function in time is obviously wrong. In general the RWA fails whenever the true solution has either weakly decaying Fourier components or a maximum of $A_{k n}$ with respect to $k$ for some value of $k>1$. We will see that this can happen to discrete breathers. In principle the RWA can be extended by increasing $k_{0}$ (in [90] this is demonstrated using a potential with barriers instead of (2.11), for which the $k_{0}=2$ RWA fails when the energy of the solution is close to the barrier), and indeed the RWA then goes over into what is called numerical calculation of discrete breathers up to machine precision. The typical case $k_{0}=2$ however is again missing the problem of resonances of higher harmonics with the linear spectrum. Thus the most reliable results can be achieved with RWA when the frequency of the discrete breathers is located above the linear spectrum. Numerical solutions of the RWA equations have been obtained e.g. using the Gauss procedure [61].

\section{Numerical evidence}

Finally we want to shortly mention a number of papers demonstrating the existence of breather-like objects through numerical integrations of the equations of motion. This is achieved using either Runge-Kutta methods or Verlet (leapfrog) algorithms [149. The results are typically presented as some snapshots of the amplitude or energy distribution in the lattice during the actual integration [173], [36], 37], 38], 39], 122], 117], [35], 1170, 174], 109], [108], [107], [106], [9], [7], [51], [110], [197]. The findings strongly suggest that there exist solutions of the lattice dynamics which appear to be similar to breather solutions. Moreover Burlakov, Kisilev and Pyrkov [36] have numerically obtained breatherlike structures for a two-dimensional lattice, which implies that discrete breathers are supported by lattice dimensions one and two at least. Thus although most of the analysis in the literature has been done for one-dimensional lattices, it is very important to keep in mind that discrete breather existence is not restricted to one-dimensional systems. For some systems these solutions even move - despite the discreteness of the system. All those studies of course do not answer the question as to whether the observed objects correspond to exact solutions or not. Still it is tempting to conjecture the existence of discrete breathers, because the variety of systems analyzed numerically makes the opposite statement difficult to believe. 


\section{THE LOCAL ANSATZ}

In this chapter we will discuss numerical efforts of characterizing the breather-like objects which appear in many simulations (cf. preceeding section). These studies have been performed for one- and two-dimensional lattices. These studies generate a 'local integrability' picture, which has been useful in proceeding with the understanding of the properties of discrete breathers. For a complete discussion see Refs. [87, [90], 84. We will finally discuss the possibility of wavelet analysis of breather-like objects [106]. Note that a number of sophisticated numerical studies aimed more at the 'interaction' of discrete breathers with other objects - like discrete breathers again, or impurities etc - will be discussed later in the appropriate chapters of this review.

\section{A. A numerical experiment}

Consider a Hamiltonian which yields a more general form of equation (2.3):

$$
H=\sum_{l}\left(\frac{1}{2} \dot{u}_{l}^{2}+V\left(u_{l}\right)+\Phi\left(u_{l}-u_{l-1}\right)\right) \text {. }
$$

We specify the potential terms in (3.1) in form of an expansion around this groundstate:

$$
\begin{aligned}
& V(z)=\sum_{k=2}^{\infty} \frac{1}{k} v_{k} z^{k}, \\
& \Phi(z)=\sum_{k=2}^{\infty} \frac{1}{k} \phi_{k} z^{k} .
\end{aligned}
$$

A specific realization is $v_{2}=1, v_{3}=-1, v_{4}=1 / 4, \phi_{2}=0.1$, with all other expansion coefficients being zero. The number of lattice sites $N$ is $N=3000$. Periodic boundary conditions are used. Choose an initial condition for a numerical integration which is spatially localized: all particles at rest in their groundstate position, and one (central) particle displaced from the groundstate position by some amplitude, having zero velocity. Define the discrete energy density

$$
e_{l}=\frac{1}{2} \dot{u}_{l}^{2}+V\left(u_{l}\right)+\frac{1}{4} \phi_{2} \cdot\left[\left(u_{l}-u_{l-1}\right)^{2}+\left(u_{l}-u_{l+1}\right)^{2}\right] .
$$

The sum over all local energy densities gives the total conserved energy. If DBs are excited, the initial local energy burst should mainly stay within the DB. Thus defining

$$
e_{(2 m+1)}=\sum_{-m}^{m} e_{l}
$$

and exciting the local energy burst at lattice site $l=0$ by choosing a proper value of $m$ in (3.4) we will control the time dependence of $e_{(2 m+1)}$. If this function doesn't (or slowly enough) decay to zero, the existence of a breather-like object can be confirmed. The term 'slowly enough' has to be specified with respect to the typical group velocities of small amplitude phonons. This sets the time scale we are interested in:

$$
t \gg \frac{\sqrt{2+2 \phi_{2}}}{2 \phi_{2}}=7.416
$$

In Fig.2 (Fig.2 of [90]) we show the time dependence of $e_{(5)}$ for an initial condition $u_{0}(t=0)=2.3456, u_{l \neq 0}=0$, $\dot{u}_{l}=0$. Clearly a localized object is found. After a short time period of the order of 100 time units nearly constant values of $e_{(5)}$ are observed. The breather-like object is stable over a long period of time with some weak indication of energy radiation. The energy distribution within the object is shown in the inset of Fig.2. Essentially three lattice sites are involved in the motion, so we find a rather localized solution. While the central particle performs large amplitude oscillations, the nearest neighbours oscillate with small amplitudes. All oscillations take place around the groundstate. 


\section{B. The internal dynamics}

We show a FOURIER transformation of the motion of the central particle in the breather-like object (Fig.2 of [87]) in Fig.3 . We see clearly that there are two frequencies determining the motion of the central particle $\omega_{1}=0.822, \omega_{2}=$ 1.34. All peak positions in Fig.3 can be explained through linear combinations of these two frequencies (similar observations were found recently in 60]. To proceed in the understanding of the phenomenon, we plot in the inset in Fig.3 the FOURIER transformation of the motion of the nearest neighbour(s) to the central particle. As expected, we not only observe the two frequency spectrum, but surprisingly the peak with the highest intensity is not at $\omega_{1}$ as for the central particle, but at $\omega_{2}$. It looks like every particle has its major frequency. Because of the symmetry of the initial condition the two nearest neighbours move in phase. Thus we are left with an effective 2 degree of freedom problem (cf. inset in Fig.2).

\section{The reduced problem}

Now it is a small step to recognize, that we might be confronted with a kind of integrability phenomenon. Indeed, fixing the rest of the particles at their groundstate positions reduces the dynamical problem to a two degree of freedom system, which might be integrable in parts of its phase space:

$$
\begin{aligned}
\ddot{u}_{0} & =-V^{\prime}\left(u_{0}\right)-2 \phi_{2}\left(u_{0}-u_{ \pm 1}\right) \\
\ddot{u}_{ \pm 1} & =-V^{\prime}\left(u_{ \pm 1}\right)-\phi_{2}\left(u_{ \pm 1}-u_{0}\right) .
\end{aligned}
$$

We will call these types of few degree of freedom systems reduced problems. In [87] a POINCARE map between the trajectory and the subspace $\left\{\dot{u}_{0}, u_{0}, u_{ \pm 1}=0, \dot{u}_{ \pm 1}>0\right\}$ has been performed for the reduced problem, where the initial conditions correspond to those of the breather-like trajectory of the extended lattice. The same map has been then performed in the extended lattice itself, and the two results were compared. Not only was the existence of regular motion on a twodimensional torus found in both cases, but the tori intersections for the reduced and full problems were practically identical [87]. Thus we arrive at two conclusions: i) the breather-like object corresponds to a trajectory in the phase space of the full system which is for the times observed practically embedded on a two-dimensional torus manifold, thus being quasiperiodic in time; ii) the breather-like object can be reproduced within a reduced problem, where all particles but the central one and its two neighbours are fixed at their groundstate positions, thereby reducing the number of relevant degrees of freedom.

With these properties in mind, it is clear, that there have to appear two frequencies in the Fourier spectrum. If the phase space flow of the reduced problem is regular in some parts of the phase space, there should appear two actions $I_{n}, n=1,2$ as functions of the original variables, so that the Hamiltonian of the reduced problem can be expressed through the two action variables only, and these actions become integrals of motion. The corresponding two frequencies

$$
\omega_{n}=\frac{\partial H}{\partial I_{n}}
$$

determine the motion of system on the surface of the torus. Obviously all linear combinations of multiples of these frequencies appear in the FOURIER spectrum of the original particle displacements. That is exactly what we observe. The conclusions from above imply another consequence - namely, that a spatially asymmetric breather-like object (with respect to the central particle) should also exist, i.e. that the two nearest neighbours perform not-in-phase motions, even with different amplitudes. That would mean, that in the language of actions we lift a degeneracy by choosing asymmetric initial conditions and have to expect three instead of two fundamental frequencies, i.e. the frequency $\omega_{2}$ splits into two frequencies $\omega_{2} \neq \omega_{3}$. This has been checked in 90 in a simulation with an asymmetric initial condition, which differs from the previous symmetric initial condition by additionally choosing $u_{1}(t=0) \neq 0$.

Indeed it was found i) that the local asymmetry is conserved throughout the evolution of the system, and as the FOURIER spectrum of the central particle motion and the two nearest neighbours motions show, there are three frequencies: $\omega_{1}=0.83, \omega_{2}=1.32$ and $\omega_{3}=1.35$ [87].

\section{A correspondence conjecture}

Intuitively it is evident, that none of the observed frequencies describing the dynamics of the local entity should resonate with the linear spectrum, since one expects radiation then, which would violate the assumption that the 
object stays local without essential change. In truth the conditions are much stricter, which we will discuss later. Since the reduced problem defined above can not be expected to be integrable, we expect the typical phase space pattern of a nonintegrable Hamiltonian system with two degrees of freedom of having some regular islands filled with nearly regular motion (tori) embedded in a 'sea' of chaotic trajectories. Chaotic trajectories have continuous (as opposed to discrete) Fourier spectra (with respect to time), and so we should always expect that parts of this spectrum overlap with the linear spectrum of the infinite lattice. Thus chaotic trajectories of the reduced problem appear not as candidates for breather-like entities. The regular islands have to be checked with respect to their set of frequencies. If those frequencies are located outside the linear spectrum of the infinite lattice, we can expect localization - i.e. that a trajectory with the same initial conditions if launched in the lattice will essentially form a localized object. Islands which do not fulfill this nonresonance criterion should be rejected as candidates for localized objects. Thus we arrive at a selection rule for initial conditions in the lattice by studying the low-dimensional dynamics of a reduced problem! This conjecture has been successfully tested in [90]. In Fig.4 we show a representative Poincare map of the reduced problem. In Fig. 5 the time dependence of the above defined local energy $e_{(5)}(t)$ is shown for different initial conditions which correspond to different trajectories of the reduced problem. Clearly the initial conditions of regular islands 1,2 of the reduced problem yield localized patterns in the lattice, whereas regular island 3 and the chaotic trajectory if launched into the lattice lead to a fast decay of the local energy due to strong radiation of plane waves. It is interesting to note that the energy decay of these objects stops around $e_{(5)}=0.35$. In [90] it was noted that the fraction of chaotic trajectories in the reduced problem is practically vanishing for energies below that value. This aspect is not yet fully understood.

Another observation which comes from this systematic analysis is that the fixed points in the Poincare map of the reduced problem (in the middle of the regular islands in Fig.4) correspond to periodic orbits. A careful analysis of the decay properties as shown in Fig.5 has shown that all objects were slightly radiating - but some stronger and some less. The objects corresponding to the periodic orbits of the regular islands 1,2 of the reduced problem showed the weakest decay. Thus we arrive at the suggestion that time-periodic local objects could be free of any radiation - i.e. be exact solutions of the equations of motion of the lattice! It makes then sense to go beyond the approximations of discrete breathers as described in the previous section and to look for a way of understanding why discrete breathers can be exact solutions of the dynamical equations - provided they are periodic in time. Further the question arises, why their quasiperiodic extensions appear to decay - i.e. why do quasiperiodic discrete breathers seem not to exist. We can also ask: suppose quasiperiodic DBs do not exist - what are then their patterns of decay; what about their life-times; what about moving DBs (certainly they can not be represented as time-peiodic solutions)? Much of what follows will be finding answers to the questions raised here.

Two things have to be said before we can proceed. First the linear spectrum of the model used for the numerical results here is optical-like, with a ratio of the band width to the gap of about $1 / 10$. However this does not imply that the discrete breathers are merely due to some weakness of the interaction. First of all we will see later, that the degree of localization of DBs is not simply related to some bandwidth of the linear spectrum. Secondly we know that DBs exist for systems without on-site potentials $V(z)$ but with nonlinear interactions. Third an estimation of the energy part stored in the interaction of the DB object presented here yields a value of 0.4 . Compare that to the full energy $E \approx 0.7$. Roughly half of the energy is stored in the interaction! that shows how misleading the intuition can be. Another important addendum is that of course the local ansatz was tested for different model realizations (for details see [90]). Apparently the whole scenario is rather independent of the choice of the model!

\section{E. Two-dimensional lattice}

We left one question to be answered, i.e. - what is the impact of the dimension of the lattice? The answer seems to be - not much. This must be so because the DBs can be described by local few-degree-of-freedom systems (reduced problem). Then the only impact the dimension of the lattice will have is to increase the number of nearest neighbours, which implies simply some rescaling of the parameters of the reduced problem. To see whether that happens, the local ansatz was carried out in a two-dimensional analog of (2.7). The interested reader will find details in [84]. Here we shorten the story by stating that practically the whole local ansatz can be carried through in the two-dimensional lattice. An analog of Fig.2 for the two-dimensional case is shown in Fig.6 where the energy distribution in a discrete breather solution is shown, and the inset displays the time dependence of a local energy similar to $e_{(5)}(t)$.

The success of this step $d=1 \rightarrow d=2$ implies that nothing terrible happens if we proceed to $d=3$ - which brings us to the possibility of obtaining discrete breathers in the generic physical case (which is $d=3$ ). Certainly it becomes worthwhile to continue our studies now, since the expectations of finding some new interesting physical phenomenon increase. Note how far we proceeded as compared to the PDE studies mentioned in the introduction. 


\section{F. Wavelet analysis}

Before proceeding with the study of discrete breathers, it is worthwhile to mention a numerical technique which is capable of efficiently tracking discrete breathers. If we ask for an efficient encoding of a spatially localized entity (forget about the time-dependence for a moment), then it is certainly not a spatial Fourier series, because many normal modes will be 'excited'. A simple basis is the lattice itself (a local basis), but only as long as the discrete breather is localized on a few lattice sites (call it a 'microscopic' breather). A 'macroscopic' breather could be then described by a set of normal modes (whose number should decrease with increasing size of the breather). What about a 'mesoscopic' breather? The method of wavelets could be a good candidate to fill this window. As shown by Hori [106] it takes only a few wavelet modes to encode a breather which is neither localized on a few sites nor too extended. This can be very useful when monitoring certain patterns of a numerical integration.

\section{LOCALIZATION PROPERTIES OF DISCRETE BREATHERS}

A natural way to proceed is to use an ansatz for the analytic form of a discrete breather and to test what restrictions apply to the parameters of the ansatz. Up to now we have seen that discrete breathers are most probably regularly evolving in time, so we will use a quasiperiodic function of time. This will bring us to necessary existence conditions for discrete breathers, will explain why in general discrete breathers can be only periodic in time, but not quasiperiodic, and will allow us to estimate the decay of Fourier coefficients (which are the parameters of the ansatz) in space and in $k$-space. For details see [76], [78].

\section{A. The ansatz for a breather solution}

As we have seen in the previous section the breather-like objects evolve regularly in time, so that we choose an ansatz which yields a quasiperiodic evolution of all variables:

$$
u_{l}(t)=\sum_{k_{1}, k_{2}, \ldots, k_{n}=-\infty}^{+\infty} A_{l k_{1} k_{2} \ldots k_{n}} e^{i\left(k_{1} \omega_{1}+k_{2} \omega_{2}+\ldots+k_{n} \omega_{n}\right) t} .
$$

We will use the notations

$$
\vec{\omega}=\left(\omega_{1}, \ldots, \omega_{n}\right), \vec{k}=\left(k_{1}, \ldots, k_{n}\right)
$$

The localization property of (4.1) is defined by the boundary condition

$$
\left.A_{l k_{1} k_{2} \ldots k_{n}}\right|_{l \rightarrow \pm \infty} \rightarrow 0
$$

Since the $u_{l}(t)$ are real-valued we can reduce the number of independent Fourier coefficients $A_{l k_{1} k_{2} \ldots k_{n}}$ by demanding $A_{l k_{1} k_{2} \ldots k_{m} \ldots k_{n}}=A_{l k_{1} k_{2} \ldots-k_{m} \ldots k_{n}}$ for any $m$. Inserting (4.1) into the equations of motion for $u_{l}(t)$ and eliminating $t$ by sorting exponentials with same exponents we will arrive at some infinite number of coupled algebraic equations for the Fourier coefficients $A$, where the control parameters are the model parameters of the Hamiltonian and the frequencies $\omega_{1} \ldots \omega_{n}$. These equations will have a very complicated form due to the nonlinearities in the equations of motion. Because we consider nearest neighbour interaction (3.1) we can formally write down the resulting set of equations as a nonlinear map:

$$
M_{l+1, \vec{k}}=F\left(\left\{M_{l, \overrightarrow{k^{\prime}}}\right\},\left\{M_{l-1, \overrightarrow{k^{\prime \prime}}}\right\}\right)
$$

Here we introduced a function $M_{l, \vec{k}}$ which is defined on a discrete $n$-dimensional lattice. The lattice is given by all combinations of $\left\{k_{1}, k_{2}, \ldots, k_{n}\right\}$ where each integer $k_{n^{\prime}}$ varies from $-\infty$ to $+\infty$. We have

$$
M_{l, \vec{k}}=A_{l k_{1} k_{2} \ldots k_{n}}
$$

Let us study (4.3) in the tails of the DB, i.e. for $l \rightarrow \pm \infty$ where (4.2) holds by assumption. In the generic case $v_{2}$ and $\phi_{2}$ from $3.2,(3.3$ will be nonzero. Then we can linearize the mapping (4.3):

$$
M_{l+1, \vec{k}}=\left(\kappa_{\vec{k}}(\vec{\omega})+2\right) M_{l, \vec{k}}-M_{l-1, \vec{k}}
$$


Here we have introduced another function on the $n$-dimensional discrete space which is given by

$$
\kappa_{\vec{k}}(\vec{\omega})=\frac{v_{2}-(\vec{k} \cdot \vec{\omega})^{2}}{\phi_{2}}
$$

Equation (4.5) is linear and thus every component of $M$ in the $n$-dimensional discrete space decouples in this equation from all other components. In fact (4.5) is a linear two-dimensional map, and $M_{l, \vec{k}}=0$ is a fixed point of this map (as it is for the original nonlinear map (4.3)). It is characterized by the matrix $G$ :

$$
G=\left(\begin{array}{rr}
1 & \kappa_{\vec{k}}(\vec{\omega}) \\
1 & 1+\kappa_{\vec{k}}(\vec{\omega})
\end{array}\right)
$$

For the eigenvalues of $G$ we find

$$
\begin{aligned}
\lambda_{1,2} & =1+\frac{\kappa_{\vec{k}}(\vec{\omega})}{2} \pm \sqrt{\left(1+\frac{\kappa_{\vec{k}}(\vec{\omega})}{2}\right)^{2}-1} \\
\lambda_{1} \lambda_{2} & =1
\end{aligned}
$$

We can consider three cases:

$$
\text { a) } \kappa_{\vec{k}}(\vec{\omega})>0: 0<\lambda_{2}<1
$$

i.e. $\lambda_{2}$ is real. Especially $\lambda_{2}\left(\kappa_{\vec{k}}(\vec{\omega}) \rightarrow 0\right) \rightarrow 1$ and $\lambda_{2}\left(\kappa_{\vec{k}}(\vec{\omega}) \rightarrow \infty\right) \rightarrow 0$.

$$
\text { b) } \kappa_{\vec{k}}(\vec{\omega})<-4:-1<\lambda_{1}<0
$$

i.e. $\lambda_{1}$ is real. Especially $\lambda_{1}\left(\kappa_{\vec{k}}(\vec{\omega}) \rightarrow-\infty\right) \rightarrow 0$ and $\lambda_{1}\left(\kappa_{\vec{k}}(\vec{\omega}) \rightarrow-4\right) \rightarrow-1$.

$$
\text { c) }-4 \leq \kappa_{\vec{k}}(\vec{\omega}) \leq 0:\left|\lambda_{1}\right|=\left|\lambda_{2}\right|=1
$$

i.e. $\lambda_{1,2}$ are complex conjugated numbers on the unit circle. Consequently in cases a) and b) the fixed point of the mapping is a hyperbolic one, i.e. there exists exactly one direction (eigenvector) in which the fixed point can be asymptotically reached after an infinite number of iterations. In case c) the fixed point is a marginally stable elliptic point, i.e. starting from any direction the fixed point can be never reached after an infinite number of steps, instead

the mapping will produce a (deformed) circle around the fixed point. Thus we find that case c) (4.12) contradicts the localization condition (4.2).

\section{B. Existence conditions for time-periodic discrete breathers}

Let us consider $n=1$. Then the DB solution is periodic (cf. (4.1)). Equation (4.6) can be simplified to

$$
\kappa_{\vec{k}}(\vec{\omega})=\frac{v_{2}-k_{1}^{2} \omega_{1}^{2}}{\phi_{2}}
$$

The frequencies $\omega_{q}$ for small-amplitude plane waves around the considered groundstate of (3.1) (where $q$ is the wave number) are related to the parameters $v_{2}$ and $\phi_{2}$ by

$$
v_{2} \leq \omega_{q}^{2} \leq v_{2}+4 \phi_{2}
$$

Then it follows that case c) given in (4.12) is identical with

$$
\text { c) } k_{1}^{2} \omega_{1}^{2}=\omega_{q}^{2}
$$

We find that a time-periodic discrete breather can not exist if any multiple of its fundamental frequency equals any frequency of the linear spectrum. The reason is that we can not satisfy (4.15) and (4.2) simultaneously because of (4.12). One can interpret (4.15) as a definition of nonexistence bands on the $\omega_{1}$-frequency axis for time-periodic discrete breathers. Introducing a normalized frequency $\tilde{\omega}_{1}=\omega_{1} / \sqrt{v_{2}}$ and normalized interaction $\tilde{\phi}_{2}=\phi_{2} / v_{2}$ (only if $\left.v_{2} \neq 0\right)$ those bands are given by 


$$
\frac{1}{k_{1}^{2}} \leq \tilde{\omega}_{1}^{2} \leq \frac{1+4 \tilde{\phi}_{2}}{k_{1}^{2}}
$$

For frequencies $\omega_{1}$ below the linear spectrum $\omega_{q}$ these nonexistence bands increase in width with increasing $\tilde{\phi}_{2}$ and completely overlap at $\tilde{\phi}_{2}=3 / 4$. Thus time-periodic DBs with frequencies below the linear spectrum should only exist if the band width of the linear spectrum is narrow enough. On the contrary no frequency restrictions seem to apply for the existence of single frequency DBs if the frequency is located above the linear spectrum.

Now let us make some statements about spatial correlations of phases of periodic DBs if they exist. If the frequency of the DB is above the linear spectrum then it follows $\kappa_{\vec{k}}(\vec{\omega})<-4$ for all $k_{1}$. This corresponds to case b) in (4.11). Then we have $-1<\lambda_{1}<0$. Consequently $-1<M_{l+1, \vec{k}} / M_{l, \vec{k}}<0$ for all $l$ in the tail of the DB. Thus we expect a coherent out-of-phase type of the motion of neighbouring particles in the tails of the DB. If the frequency of the periodic DB is below the linear spectrum $\left(v_{2}>0\right)$ things become more complicated. Namely there will always exist a certain finite integer $k_{c}$ such that for $k_{1}<k_{c}$ it follows $k_{\vec{k}}(\vec{\omega})>0$ which corresponds to case a) in (4.10). The corresponding Fourier components would yield in-phase type of motion in the tails of the DB. However for all $k_{1}>k_{c}$ the case b) in (4.11) applies. Those Fourier components would yield out-of-phase motion. Thus we have to expect a complicated mixture of in- and out-of-phase type of motion.

\section{Quasiperiodic discrete breathers}

Let us consider $n=2$. Then case c) in (4.12) applies if

$$
v_{2} \leq\left(k_{1} \omega_{1}+k_{2} \omega_{2}\right)^{2} \leq v_{2}+4 \phi_{2}
$$

Now it is possible to show that there exists an infinite number of pairs of the integers $\left(k_{1}, k_{2}\right)$ such that (4.17) is satisfied if the ratio $\omega_{1} / \omega_{2}$ is irrational and $v_{2} \geq 0$ and $\phi_{2}>0$ (cf. Appendix in [76]). Thus we can not expect the existence of two-frequency DBs. The argument for $n \geq 3$ is also straightforward and yields the same result.

Some comments are appropriate at this stage. If we consider the breather solution (1.2) of the sG PDE, then its decomposition into Fourier components (which are smooth functions of $x$ ) can be also done. But then why does the sG breather exists at all in the presence of an infinite number of resonances of multiples of the breather's frequency with the linear spectrum? This undoubtly is a subtle question. We will try to give a qualitative explanation. Writing down the differential equations for the Fourier amplitudes with all nonlinearities yields for each amplitude a differential equation with homogeneous and inhomogeneous terms. Say we linearize the equations in the tails of the breather solution. Suppose we did that for a Fourier component not in resonance with the linear spectrum. Then the linearized equations give us a one-parameter manifold of solutions contracting to zero if integrated to infinity, with exponential falloff. Did we miss something by neglecting the nonlinear inhomogeneous terms? It could be, that the inhomogeneous terms decay slower than the homogeneous solution. Then we know that the inhomogeneous part will be the leading one if integrating far away from the center. Still we are not restricted in the choice of the homogeneous solution we still have a one-parameter family of solutions. Take now a Fourier component which resonates with the linear spectrum. Then the linearized equation yields oscillating solutions. The only way to avoid that is to choose the homogenenous solution to be zero - thus loosing the one-parameter manifold of solutions. If now the inhomogeneous nonlinear parts omitted by the linearization will show up with exponential decay, the full solution will decay in space too - but it ceases to be a one-parameter manifold of solutions, it is just one fixed solution. This is precisely what happens to the sG breather solution (a nice calculational exercise!). Suppose we change the Hamiltonian slightly i.e. we change the control parameters defining the differential equation or the difference equations discussed in this review - then any solution should stay a solution, simply change slightly. But if we had a resonance, this will have a dramatic effect - we will in general slightly change the homogenenous solution (from zero to nonzero) and thus loose the breather, instead obtaining a breather-like object which is however not fully decaying to zero in the tails! This is nothing else but Boyd's nanopteron [34], which Kivshar and Turitsyn coin soliton on a standing carrier wave [130].

This should make clear that any resonance is deadly for the breather - either periodic or quasi-periodic in time. Exceptions can occur, but will be nongeneric. So is the sG breather for PDEs, so are quasi-periodic DBs (or timeperiodic DBs with resonances). Another way of explaining it is that in the case of resonance(s) the additional requirement of putting the mentioned homogeneous solutions to zero makes the set of equations for the Fourier components overdetermined, and thus solvable only for special cases (careful: this type of argument is applicable if one considers only solutions which do decay in the tails to zero!).

Cai, Bishop and Grønbech-Jensen [40] have demonstrated that quasiperiodic DBs exist for the Ablowitz-Ladik lattice. This is one of the mentioned exceptions. Not only is the AL lattice integrable (note that there is no direct connection between integrability and the existence of breathers) but it is very restrictive with respect to the equations 
for the Fourier amplitudes. We have checked that the equations for the Fourier amplitudes allow for solutions when all Fourier amplitudes with say negative Fourier numbers $k$ are zero (the variables here are complex). Consequently this puts a tremendous constraint on the combinations of $\left(k_{1}, \ldots, k_{n}\right)$ for which resonances with the linear spectrum can appear - in fact it is easy to see that for the choosen solution in [40] no resonance appears at all!

\section{The spatial decay of DBs}

From now on discrete breather implies a time-periodic spatially localized solution. Let us assume that such a solution is given, and analyze its localization properties. For that we will first linearize the equations of motion in the tails and solve for each Fourier component. The result is an exponential decay given by the eigenvalues (4.8):

$$
A_{k l} \sim\left(\operatorname{sgn}\left(\lambda_{k}\right)\right)^{l} \mathrm{e}^{l n\left|\lambda_{k}\right| l},
$$

where the eigenvalue choosen is the one with absolute value lower than one. Note that (4.8) depends only on the linear parameters defining the linear spectrum. Consequently all one has to know about the system in order to predict the spatial decay are the frequency of the DB and the position of the linear spectrum defined by $\left(v_{2}, \phi_{2}\right)$. In Fig.7 the dependence of the Fourier amplitudes $A_{k l}$ on $l$ is shown for a solution obtained in [76] (we will discuss the method of obtaining these solutions later). An exponential decay of the $A_{k l}$ is obtained, with $k$-dependent exponents. Comparing the numerically obtained exponents with the prediction of the linearization (4.18) we find excellent agreement in Fig.8. Another prediction we can formulate is, that if the DB frequency $\omega_{1}$ is restricted to be in the nonzero gap of the linear spectrum, then there exists a nonzero value of $\omega_{1}^{(m)}$ such that the exponential decay in the DB tails will be weaker for all other frequencies $\omega_{1} \neq \omega_{1}^{(m)}$ (still belonging to the gap). Let us explain why this statement follows from the previous considerations. First the frequency $\omega_{1}$ has to be larger than the linear spectrum width - else one (or more) of multiples of $\omega_{1}$ will always lie in the linear spectrum. Secondly if $\omega_{1}$ is slightly below the lower edge of the linear spectrum, then the spatial decay of the $k=1$ component will be very weak. Lowering $\omega_{1}$ we increase the decay exponent for $k=1$, but since $2 \omega_{1}$ comes closer to the upper band edge of the linear spectrum, there will be a certain frequency when the decay in the tails will be governed by the second harmonic rather than the first harmonic of $\omega_{1}$. A calculation of $\min \left(1-\left|\lambda\left(\kappa_{k}\right)\right|\right)$ with $\left|\lambda\left(\kappa_{k}\right)\right| \leq 1$ for different $\omega_{1}$ has been performed in [76] (Fig.2 of [76]). A comparison of the positions of the observed maxima with numerical simulations which yielded whole families of DBs for different frequencies clearly demonstrated the validity of the prediction - the DBs with frequencies in the gap can not be infinitely strongly localized, thus there has to be a DB solution that shows up with the strongest spatial localization. Note that for DBs with frequencies above the linear spectrum nothing similar occurs - the Fourier components with larger $k$ are always stronger localized than those with smaller $k$ (an exception is the $k=0$ component, which we will discuss later).

\section{E. Nonlinear corrections}

As already discussed, the linearization procedure fails whenever nonlinear inhomogeneous terms decay more weakly in space than the linear homogeneous solution. This will happen typically whenever any of the multiples of the DB's frequency comes close to the linear spectrum. Note that this includes the limit when the DB's frequency is itself very close to the linear spectrum - a limit where several perturbation theories are usually applied. Consequently the findings of this subsection are of relevance also for DB solutions with frequencies above the linear spectrum.

Let us define the function $d(k)=\ln \left(\left|\lambda_{k}\right|\right)$, which is by definition always negative. Consider $k \geq 0$. Then $d(k)$ has a single maximum for some $k=k_{m}$ and is monotonically decreasing with further increasing $k$. The linearization procedure is violated for a given $k_{0}$ if there exists a sequence of $k_{1}, k_{2}, \ldots, k_{\alpha-1}$ (where either $v_{\alpha} \neq 0$ or $\phi_{\alpha} \neq 0$ ) such that

$$
d\left(k_{0}\right) \leq\left(d\left(k_{1}\right)+d\left(k_{2}\right)+\ldots+d\left(k_{\alpha-1}\right)\right)
$$

holds together with the condition

$$
k_{0}= \pm k_{1} \pm k_{2} \pm \ldots \pm k_{\alpha-1} .
$$

It follows that the linearization is always correct for $k_{m}$. In [78] it was shown that the number of other $k$ for which the linearization does not hold is always finite for finite distance of $\omega_{1}$ from the linear spectrum. A numerical test has verified the correctness of the above statement, and in [78] quantitative agreement has been found (one can predict the corrected exponents of the spatial decay of the affected components by finding the leading inhomogeneous term) between numerical DB solutions and the theoretical prediction. 


\section{F. The decay in $k$-space}

Because higher harmonics are at large distance from the linear spectrum, the corresponding Fourier components decay much faster in space. That also implies that the decay in $k$ is increased when increasing the distance from the breather's center [78]. Using the linearization procedure Flach obtained for large $k$ [78]

$$
\left|A_{k l}\right| \sim k^{-2|l|} s(k)
$$

where $l$ measures the distance from the DB center and $s(k)$ is some site-independent function (typically decreasing exponentially with increasing $k[78$ ). Thus hopping from one lattice site to the neighbour site increases the decay in $k$-space by multiplying the original decay function with a power $1 / k^{2}$ ! The numerical DB solution analysis yields perfect agreement to machine precision (Figs.7,8 in [78]).

\section{G. The case $v_{2}=0$}

So far we discussed cases when $\phi_{2} \neq 0$ and $v_{2} \neq 0$. The linear spectrum is in that case optical-like, i.e. it has a nonzero lower bound and of course a finite upper bound. Let us see what to expect when this is not the case anymore.

First of all in order to keep the groundstate solution of the system we have to demand $v_{3}=0$ then without loss of generality. The same applies to the later discussed case $\phi_{2}=0$ of course.

The linear spectrum still exists, but it is acoustic-like - i.e. the lower bound is zero. Nothing spectacular will happen then for all Fourier components with $k \neq 0$. However the $k=0$ component (the dc component) will resonate with the lower bound of the linear spectrum! We are saved if this dc component is zero by some symmetry reason e.g. for all $\Phi(z)=\Phi(-z)$ and $V(z)=V(-z) A_{k l}=0$ for all even $k$, including the dc term. What if we change the interaction potential and loose this symmetry? Numerical simulations [21], [45], [110, 118, 1119], [121], [120], 1156] have shown that for one-dimensional systems of that type (with the additional restriction $V(z)=0$ which can be called a Fermi-Pasta-Ulam (FPU) lattice) a breather-kink state forms, i.e. the Fourier components with $k \neq 0$ decay in space to zero whereas the dc component shows up with a kink-like structure. Assuming that these numerically found objects correspond to solutions with finite energy, we can immediately obtain the spatial variation of the $k=0$ component. Because far away from the solution the dc displacements can be viewed as a strain field caused by a point-like source (in full analogy with the coresponding Maxwell equation for the electric field, see [133]) it follows that the decay can be calculated using Gauss's theorem. For a $d$-dimensional system the decay of the dc component should be then $\sim 1 / r^{d-1}$ [80]. So for a one-dimensional lattice we indeed obtain no decay at all, which corresponds to the mentioned numerical findings of a kink structure. In two- and three-dimensional lattices the prediction would be an algebraic decay $1 / r$ and $1 / r^{2}$ correspondingly. That could affect the exponential decay of the $k \neq 0$ components by changing the exponential decay to an exponential times an algebraic decay. Numerical tests are needed in order to check these possibilities.

Clearly if $v_{\mu} \neq 0$ with $\mu \geq 4$ then even for $d=1$ a kink-like structure would be impossible together with the decay of all other Fourier components. Solving the equations for the dc component and taking the smallest $\mu$ for which $v_{\mu} \neq 0$ we can predict $A_{0 l} \sim l^{-2 /(\mu-1)}$ for $d=1$. Since $\mu \geq 4$ here this decay is either $1 /\left(l^{2 / 3}\right)$ or weaker. Altogether the issue of the dc component resonating with the linear spectrum is an issue which requires further investigation.

\section{H. Systems without a linear spectrum}

Consider $\phi_{2}=0$. This implies that the linear spectrum is highly degenerated and given by one value $\sqrt{v_{2}}$. We do not have problems with resonances here (provided $v_{2} \neq 0$ or $V(z), \Phi(z)$ are even functions), so DBs will exist. Moreover the decay of the Fourier components will be faster than exponential in the tails. Sorting the equations for the Fourier components we will obtain in lowest order $\omega_{1}^{2} k^{2} A_{k l} \sim A_{k, l-1}^{\mu-1}$ where $\mu$ is the smallest even integer for which $\phi_{\mu} \neq 0$ (note that the interaction has to be nonzero - otherwise we solve the trivial problem of uncoupled particles). The resulting decay is

$$
A_{k l} \sim \mathrm{e}^{(\mu-1)^{l} a_{k}}
$$

where $a_{k}$ is some number which depends on $k$. The result (4.21) is an exponential decay with the variable being not the distance itself but rather an exponential function of the distance again! A nice example to test this is the FPU lattice in one dimension with $\phi_{2 m} \neq 0$ and all other control parameters of the potentials vanishing. The amplitudes obtained e.g. in [76] or in [14] fit the predicted spatial decay. 
It is not clear at present what happens if $v_{2}=0$ and the potential functions are not even. Clearly these problems are waiting for an answer.

\section{Higher lattice dimensions}

In this section we studied DBs for one-dimensional systems. The generalization to higher lattice dimensions is straightforward. Instead of solving linear maps one has to introduce Green's functions [169], [51]. The results will be essentially the same. Especially quasiperiodic DBs will again turn out to be nongeneric, and time-periodic DBs will exist if all multiples of their frequency do not resonate with the linear spectrum.

\section{EXISTENCE PROOFS}

In this section we will discuss existence proofs for discrete breathers - not for isolated model systems (like the Ablowitz-Ladik one) but for classes of systems. The central part in this section will be the continuation of periodic orbits - starting with the limiting case of uncoupled particles (e.g. $\Phi(z)=0)$ and then continuing to finite interaction. This approach has been coined antiintegrability method by Aubry [12], which is connected to the coding sequence structure which defines a certain periodic orbit of the noninteracting many-particle system. The proof has been performed by MacKay and Aubry [137], and with some modifications also by Bambusi [16]. Further we will discuss the proof of existence of DBs for systems with homogeneous potentials by Flach [77, which works especially for systems with $V(z)=0$ where the antiintegrability method is not applicable directly. Finally we will discuss some related results obtained in the mathematical literature. Only the essential ideas of the proofs and the conditions for their application are given here. For a more complete discussion see Refs. [137], [16], [77], [13].

\section{A. The antiintegrability approach}

The concept of antiintegrability was initially introduced for the Standard map [14]. This concept turned out to be a simple and powerful tool for finding solutions of nonlinear systems. It is in essence the continuation of zeroes of an operator $F$ from a solvable limit. The nonlinearity is necessary in order to be sure that the eigenvalues of a certain Newton operator (a differential of the operator $F$ ) are well behaved for the solution to be continued. The antiintegrability method allows for a more efficient and numerically easier and reliable calculation of chaotic trajectories of nonlinear maps than other methods do. It can be easily extended to coupled maps.

Consider the model (3.1) with parametrized interaction $\Phi(z)=\epsilon \Psi(z)$. Consider the case $\epsilon=0$. Then the equations of motion are trivial since they decouple in space. All we have to do is to solve for each individual oscillator moving in its potential $V(z) \neq 0$. Note that we can not discuss $V(z)=0$ since then the particles are unbounded, which makes the following impossible. Each local solution can be then given in the form

$$
u_{n}(t)=g\left(\omega_{n} t+\alpha_{n}\right),
$$

where the function $g(t)$ is periodic in time with period $2 \pi$. The frequency $\omega_{n}$ is a unique function of the action $I_{n}$ of the $n$th oscillator (not that the reverse is not true - in general each value of $\omega_{n}$ can correspond to several isolated values of the action).

The general idea of the proof is: consider a time-periodic solution of the whole lattice for $\epsilon=0$ - e.g. one where all oscillators are at rest but one oscillator is moving (encoded with its values of the frequency $\omega_{n}$ and phase $\alpha_{n}$ ). Then it is possible to find a periodic orbit of the lattice with $\epsilon \neq 0$ but close to zero such, that the new periodic solution will have the same frequency and be close to the old periodic solution. In other words we can continue the periodic orbit of the noninteracting system to finite interactions. It can be shown then that these periodic orbits will be exponentially localized around the chosen center $n$, and thus are discrete breathers.

The method starts with considering $F(u ; \epsilon)=\left(F_{1}(u ; \epsilon), F_{2}(u ; \epsilon), \ldots\right)$ with

$$
F_{n}(u ; \epsilon)=\ddot{u}_{n}+\frac{\partial H}{\partial u_{n}} .
$$

Note that $H$ parametrically depends on $\epsilon$. We are looking for zeroes of $F(u ; \epsilon)$ (i.e. each component of $F$ must vanish) since the zeroes of $F(u ; \epsilon)$ solve the equations of motion. The goal is to apply the implicit function theorem (see e.g. [56]). For that we need a subspace of all functions $u=\left\{u_{n}(t)\right\}$ which yields isolated zeroes of $F$. This can be done 
by restricting the consideration to time-periodic functions. Still the frequency and the phase at each lattice site could be choosen in different ways, which creates smooth manifolds of zeroes of $F$. To guarantee that $F$ has only isolated zeroes for $\epsilon=0$, MacKay and Aubry demand that the frequency and phases are fixed [137] by staying in the subspace of all periodic functions $u=\left\{u_{n}(t)\right\}$ with:

$$
u_{n}(t)=u_{n}\left(t+2 \pi / \omega_{b}\right)
$$

and

$$
u_{n}(t)=u_{n}(-t)
$$

Here (5.4) is allowed because the Hamiltonian equations of motion are time reversible. Now the zeroes of $F(u ; \epsilon)$ are isolated for $\epsilon=0$. Application of the implicit function theorem is then possible. One is looking for a continuation of $u(\epsilon)$ such that $F(u(\epsilon), \epsilon)=0$. The main problem is to show that $\partial_{u} F$, the derivative with respect to $u$, is invertible at the initial solution (for $\epsilon=0$ ). The Newton operator $\partial_{u} F(u(\epsilon) ; \epsilon$ ) has to fulfill the equation

$$
\partial_{u} F(u(\epsilon) ; \epsilon) \frac{\mathrm{d} u(\epsilon)}{\mathrm{d} \epsilon}+\partial_{\epsilon} F(u(\epsilon) ; \epsilon)=0
$$

which is a differential form of $F=0$. Continuation to $\epsilon \neq 0$ can be performed provided the nonresonance condition $k \omega_{b} \neq \sqrt{v_{2}}$ and the nonlinearity condition $V(z)-\left(v_{2} / 2\right) z^{2} \neq 0$ hold. It can be continued until the Newton operator ceases to be invertible - which corresponds to a collision of Floquet multipliers at +1 in the dynamical stability analysis of the discrete breather orbit (see below). The exponential degree of localization of the discrete breather is proven in [137. which studies the properties of the inverse of the Newton operator.

The remarkable thing about this proof is that nothing has to be said neither about the lattice dimension nor about the size of the system, which can be infinite. Thus indeed discrete breathers exist e.g. in two- and three-dimensional lattices. Another interesting fact is that MacKay and Aubry can also continue 'multisite' breathers - i.e. solutions which are still exponentially localized in space but whose internal structure is far more complicated than the simple structure of the 'one-site' breathers.

Bambusi has recently provided a similar proof with the difference that the energy of the solution is fixed (continuation for constant energy rather than for constant frequency).

Using the antiintegrability approach MacKay and Aubry prove the existence of discrete breathers in the discrete nonlinear Schroedinger equation [137]. This is especially interesting because the DNLS structure is close to different models of interacting classical spins, so that one can expect classical spin breathers to exist (numerically these solutions have been found in [189, 176 using RWA).

Aubry [13] and Perfetti 146] demonstrate the existence of 'rotobreathers' for Hamiltonians which are periodic in the displacements (angles). These solutions correspond to a finite number of rotators rotating, and the rest of the rotators librating with exponentially decreasing amplitude as the distance to the center is increased. Numerical evidence of rotobreathers is reported in 178 .

\section{B. The homoclinic orbit approach}

Consider a system with homogenenous potentials $V(z)=v_{2 m} z^{2 m} /(2 m)$ and $\Phi(z)=\phi_{2 m} z^{2 m} /(2 m)$. The equations of motion become

$$
\ddot{u}_{l}=-v_{2 m} u_{l}^{2 m-1}-\left(u_{l}-u_{l-1}\right)^{2 m-1}-\left(u_{l}-u_{l+1}\right)^{2 m-1} .
$$

Note that we can always use $\phi_{2 m}=1$ by rescaling time. Kivshar [126] noted that one can look for time-periodic solutions of (5.6) in the form

$$
u_{l}(t)=A_{l} G(t)
$$

which separates time and space. Inserting (5.7) into 5.6) we obtain a differential equation $\ddot{G}(t)=-\kappa G^{2 m-1}(t)$ with a separation parameter $\kappa>0$ and a two-dimensional map for the amplitudes $A_{l}$ :

$$
\kappa A_{l}=v_{2 m} A_{l}^{2 m-1}+\left(A_{l}-A_{l-1}\right)^{2 m-1}+\left(A_{l}-A_{l+1}\right)^{2 m-1} .
$$

Since the equation for the masterfunction $G(t)$ yields time-periodic solutions, we have to show that homoclinic orbits exist for the discrete map (5.8) and will thus prove the existence of discrete breathers. This has been done by Flach in 
177 for $v_{2 m}=0$, i.e. for a system which does not fall into the class considered by MacKay and Aubry in the previous subsection. The proof can be easily extended to incorporate $v_{2 m} \neq 0$. Let us sketch the main ideas. First of all we have no restriction on $\kappa$ other than $\kappa>0$. Secondly we consider $f_{l}=\left|A_{l}\right|$ with

$$
\kappa f_{l}=v_{2 m} f_{l}^{2 m-1}+\left(f_{l}+f_{l-1}\right)^{2 m-1}+\left(f_{l}+f_{l+1}\right)^{2 m-1} .
$$

Equation (5.9) has a hyperbolic fixed point $(0,0)$ and an elliptic fixed point $(f, f)$ for $\kappa_{f p 2}=\left(v_{2 m}+2^{2 m}\right) f^{2 m-2}$.

We start with a set of two nearest neighbour values $f_{l-1}$ and $f_{l}$ and compute $f_{l+1}$ using (5.9). Requiring $0<f_{l+1}<$ $f_{l}$ we obtain an allowed region for $\kappa$, where the lower bound of this region corresponds to $f_{l+1}=0$. Repeating this procedure with increasing $l$ we find that the lower bound on $\kappa$ is monotonically increasing with $l$. Making sure that at the beginning of the map the lower bound was below $\kappa_{f p 2}$ we thus arrive at the conclusion that it will always stay below $\kappa_{f p 2}$, but it can not asymptotically reach it either for $l \rightarrow \infty$, so we will find a $\kappa$ for which the the iteration leads to a decay to zero. By proper choice of the initial pair of neighbouring values and using the inversion symmetry of (5.9) we can complete the proof.

The existence of homoclinic orbits of (5.9) also implies the existence of intersection points of the stable and unstable invariant manifolds of the hyperbolic fixed point $(0,0)$. In [77] the existence of horseshoe patterns was demonstrated. Then it is evident that the invariant manifolds will allow for a much richer set of homoclinic orbits than say the onesite breathers considered. To our understanding these rich intersection patterns of the invariant manifolds directly compare to the possibility of continuing multi-site breathers using the antiintegrability approach.

\section{NUMERICAL METHODS OBTAINING DISCRETE BREATHERS}

In this section we will briefly review some numerical methods which allow to calculate a discrete breather solution to machine precision. Thus we will not discuss here approximate methods which are not aimed at a precise calculation (like e.g. letting some initial localized conditions to evolve and to radiate away what is not belonging to the solution, or using from the beginning an approximation like RWA - thus limiting oneself much above machine precision). At the end of each part we will list the advantages and disadvantages of each method.

The choice of relevant variables separates these methods into two classes. One class corresponds to choosing initial conditions of the Hamiltonian system. Defining the frequency of the DB one numerically integrates the initial conditions over one DB period, and obtains a map of the phase space into itself. The fixed points of this map are periodic solutions with the given period. Methods operating with these variables can be efficient in programming, because one needs typically two variables per lattice site. The numerical integration over the DB period can be performed very fast using standard integration routines - $10^{4}$ lattice sites is well in the range of perfomance of a typical workstation or PC. The disadvantage of this method is that we create numerical errors already at the stage of computing the map. However the efficiency of integration routines practically eliminates these problems. The second class of methods works with the Fourier coefficients $A_{k l}$. These methods have the advantage that one is searching for solutions without leaving the subclass of periodic solutions (defined by the Fourier series). The disadvantage of this method is that the number of variables is strictly speaking infinite (because one needs an infinite number of Fourier coefficients per lattice site). In practice we will have then to define a threshold value for the Fourier number $k_{t}$ such that we neglect all Fourier coefficients with $k>k_{t}$. This can be usually done because any smooth periodic function with finite period will show up with a decay of its Fourier coefficients for large $k$. So one has to check after finding a solution whether the cutting value $k_{t}$ was justified. This is especially important because the nonlinearity in the original equations of motion produces infinite-range interactions in Fourier space! Still we will be left with more than two variables per lattice site - typically 10-30. Also the explicit form of the equations of the Fourier coefficients can be complicated, such that we will have to use integration routines in order to find the Fourier coefficients of a certain function - which in addition creates the necessity of integrations and again increases the numerical errors.

The methods to be discussed differ not only with respect to the chosen variables. Once we have the variables, we can use Newton's method, the method of steepest descent, or other nonlinear maps. The first two of them rely on continuing from a limiting model case where the solution can be approximated to good precision, some other methods do not. For an extensive discussion and technical details see Refs. [41], [78], 140], [13], 32], 31].

\section{A. Newton method}

Recall the Newton method for the problem $f(x)=0$ which is solved for some $x=s$. We choose a good approximation for the true solution $x_{(0)}$ and expand $f(x)=f\left(x_{(0)}\right)+f^{\prime}\left(x_{(0)}\right)\left(x-x_{(0)}\right)$. We thus obtain the iteration procedure $x_{(n+1)}=x_{(n)}-f\left(x_{(n)}\right) / f^{\prime}\left(x_{(n)}\right)$. Here $f^{\prime}(x)$ is the first derivative of $f$ with respect to $x$. The method converges if $x_{(0)}$ is sufficiently close to $s$ and if we do not encounter a zero in $f^{\prime}(x)$ on our iteration route. 


\section{Phase space variables}

Consider the numerical integration of the equations of motion for the lattice over a given period $T$

$$
u_{n}(T)=I_{n}(\{u\} ; T)
$$

where the integration procedure $I$ depends parametrically on $T$ and on the initial conditions $\{u\}$. The initial velocities can be chosen to be zero $\dot{u}(0)=0$. A periodic orbit of period $T$ will then lead to

$$
f_{n}=0, f_{n}=u_{n}-I_{n}(\{u\} ; T) .
$$

This is so because the kinetic energy of the system is a positive definite quadratic form of the velocities. Integrating over $T$ and finding all positions to be the same then implies that the kinetic energy is again zero - and thus the velocities are the same as the initial ones (one can easily abandon these restrictions if searching for solutions of systems without either time-reversal symmetry or some other nontrivial properties). Define $\vec{u}=\left(u_{1}, u_{2}, \ldots, u_{n}, \ldots\right)$, $\vec{f}=\left(f_{1}, f_{2}, \ldots f_{n}, \ldots\right)$ and the matrix $M$ with elements $M_{l m}=\partial f_{l} / \partial u_{m}$. Then the Newton method generates the map

$$
\vec{u}_{(n+1)}=\vec{u}_{(n)}-M^{-1} \vec{f}\left(\vec{u}_{(n)}\right) .
$$

The map converges if we are close enough with the initial guess $\vec{u}_{(0)}$ and if the Newton matrix $M$ is invertible. To obtain a good initial guess it is useful to consider some limiting case of the system - e.g. the antiintegrability limit or something else. Once a solution is found, we can change the parameters of the Hamiltonian and/or the period of the breather by small amounts and (thus assuring that we have a good guess of a new solution, namely the old one) repeat the iteration. This yields a systematic method of tracing a solution.

Typically one needs about 10-20 iterations for one solution. One disadvantage of this method appears whenever the Newton matrix is not (or nearly not) invertible. Still the breather solution can continue to exist! A second problem can be computation time. For each step in the iteration we have to perform $N$ integrations of the equations of motion of the system - over the given period $T$. This will give us the Newton matrix. Still that is not the main source of computing time consumption - its computing time grows as $N$. The calculation of the inverse of the Newton matrix can become the main source of time problems, since the computing time grows as $N^{2}$ at least.

\section{Fourier coefficients}

The choice of the Fourier coefficients as the variables of the Newton method does not change much. We have to increase the number of variables per site - typically about 10-30 per site. Still the $A_{k l}$ can be a good choice - especially if we can explicitly write down the equations (i.e. that we can explicitly encode them in a program). That implies that the nonlinear terms of the equations of motion are finite order polynomials in the displacements. This allows for an explicit dependence of the elements of the Newton matrix on the $A_{k l}$. We will need some effort to write the program code, but the final iteration will proceed very fast!

If the nonlinear terms of the equations of motion are not finite order polynomials, then one has to numerically perform a Fourier integration over the nonlinear terms, which are functions of the $A_{k l}$ [78]. This implies that we have to numerically obtain the Newton matrix.

The advantage of this method occurs whenever the equations of motion are low order polynomials in the phase space variables. Still it requires at every iteration step the computation of the inverse of the Newton matrix, which can cause computing time problems for large systems. The method will again fail whenever the Newton matrix is not invertible.

\section{B. Steepest descent}

Recall the steepest descent method for solving $f(x)=0$. We define $g(x)=f^{2}(x) \geq 0$. If $x=s$ is a root which solves $f(x)=0$ then $g(x)$ has a minimum at $x=s$ with $g(s)=0$. To find the minimum we calculate the gradient (here simply the derivative) of $g(x)$ and perform small steps in $x$ in the direction of the negative gradient. The numerical implementation needs a variable step size, which has to change whenever a subsequent step does not lower the value of the function $g(x)$. Eventually we will end in a minimum of $g(x)$ (this is signaled by the step size becoming smaller than say the precision of the machine). If our initial starting point $x$ was sufficiently close to $s$ the function $g(x)$ will be zero at the determined minimum - a signature that we have solved the problem $f(x)=0$. 


\section{Phase space variables}

Using the equations (6.1), (6.2) and the definitions from above we can generalize the function

$$
g(\vec{u})=\vec{f} \cdot \vec{f} .
$$

The gradient is given by

$$
\nabla g=2 \vec{f} \cdot(\nabla \cdot \vec{f})
$$

with the elements of the gradient given by

$$
(\nabla g)_{l}=2 \vec{f} \cdot \frac{\partial \vec{f}}{\partial u_{l}}
$$

To compute the gradient we have to perform $N$ integrations of the equations of motion (as with the Newton method). Once we have a gradient we use the negative of its direction in the variable's space to go as far as possible - under the condition that $g$ is minimized. That defines the next 'step' at which we have to compute the gradient again. To obtain a good initial guess it is again useful to consider some limiting case of the system - e.g. the antiintegrability limit or something else. Once a solution is found, we can change the parameters of the Hamiltonian and/or the period of the breather by small amounts and thus assuring that we have a good guess of a new solution, namely the old one) repeat the iteration. This yields a systematic method of tracing a solution.

The implementation of the method itself is slightly more complicated (but still a basic task for any experienced programmer) than the Newton routine - which is a simple map. However this method does not compute and inverts matrices! All we have to do is to compute gradients (vectors). This method was used for the calculation of discrete breathers in three-dimensional systems [83]. The structure of the program is in fact trivial if one splits the formal search for the minimum of $g$ from the system-specific calculation of the gradient. Another advantage of this method is that it does not break down when the Newton matrix ceases to be invertible. Consequently this method traces discrete breathers through dynamical instabilities without any problem. If at an instability (bifurcation) other breather families appear, the method will simply contract onto one of them. Combined with the Newton method this can be efficient in studying bifurcations, because the Newton method is sensitive to bifurcations, where $M$ is no longer invertible. Since the Newton matrix yields the relevant eigenvectors of perturbations, one can then use the structure of the eigenvectors and with the help of the steepest descent follow any of the new periodic orbit branches. The disadvantage of the steepest descent is that there is no guarantee that the minimum of $g$ which is always found corresponds to a zero in $g$ or better in $\vec{f}$. However we did not encounter any practical problems of that kind.

\section{Fourier coefficients}

Again nothing will change if we use Fourier coefficients instead of phase space variables. As for the Newton method this change is only reasonable if the nonlinear terms of the equations of motion are finite order polynomials in the phase space variables. Otherwise the advantages/disadvantages are not affected.

\section{Other methods}

Campbell and Peyrard [11] have also used a Newton method to generate breathers. However the choice of the variables there was quite different from the above. Namely the discretization of time which is needed in any numerical intergration, has been used in [41] in order to define a coupled map lattice in two dimensions - one discrete spatial and one discrete time dimensions. Defining appropriate boundary conditions one can indeed search for discrete breather solutions. The disadvantage of this method is that in order to keep computational time within reasonable limits one has to choose quite large time steps - in [41] $1 / 64$ of the breather's period. Compare this with typical time steps of $1 / 1000$ of the breather's period when numerically integrating the equations of motion.

There are specific cases when one can use symmetries of a system to design special methods. Take the case of homogeneous potentials as an example. The equations for the time-independent amplitudes are given in (5.8). These equations can be generated by the extrema of the function $(m \geq 2)$

$$
S=\sum_{l}\left[\frac{1}{2} A_{l}^{2}-\frac{v_{2 m}}{2 m} A_{l}^{2 m}-\left(A_{l}-A_{l-1}\right)^{2 m}\right] .
$$


Because $S$ is a positive quadratic form of the variables $A_{l}$ close to zero and a negative $(2 \mathrm{~m})$ form far from zero the only nontrivial extrema will be saddles - minima on a rim which surrounds the valley with the local minimum $A_{l}=0$. A very efficient method of computing these saddles is to choose a certain direction (which essentially encodes the breather solution one is searching for) and to find the maximum of $S$ when departing from the origin in this direction. Once we have done that, we are on the rim! Now we have to use steepest descent to minimise $S$ on the rim - by adjusting after each step the point to the rim again (in leading order we will always go down the rim, but there will be always a small deviation from the rim itself). This method will yield breather solutions extremely fast - also for higher lattice dimensions, because the method is local (if the initial direction was local of course)2

Yet another method was reported in [78] when working with Fourier coefficients $A_{k l}$. It consists of writing down each of the equations $f_{n}=0$ (see above) in the way of a map. Since there are different ways of doing that, one can choose between maps which are unstable at small amplitudes (repelling) or stable (attracting). Depending on the solution to be obtained the global map, which is a set of all the local maps, is constructed and it contains the code of the discrete breather to be searched for by encoding the map choice at each lattice site. Then one chooses a small initial condition (e.g. $A_{11}=0.1$ and all other coefficients at zero) and iterates. There is no guarantee that the iteration converges to something meaningful. Yet if it does, it happens very quickly. In [78], [17] different solutions were obtained and analyzed. The major flaw of this method is its unpredictable convergence criteria.

Neuper et al use a quasicontinuum method combined with an iterative procedure to obtain DB solutions [144]. Laedke et al use an iteration of a Fredholm type equation to both prove and numerically obtain discrete breathers in the DNLS [132].

\section{STRUCTURAL STABILITY}

In the introduction we discussed the breather solution of the sG PDE. Especially we mentioned that it is structurally unstable. What about the structural stability of discrete breathers?

Structural stability of a solution implies that if the equations generating the solution are slightly perturbed (changed) then the new equations posess a solution close to the original one - in the limit of the perturbations going to zero both solutions should coincide. This is a tricky thing - one has to be careful in defining the space of allowed perturbations (note that e.g. if we would add a small friction, then all dynamical solutions would disappear!).

That discrete breathers are structurally stable, follows essentially from the existence proof of MacKay and Aubry [137. They are structurally stable with respect to changes of the parameters of the Hamiltonian, unless a resonance with the linear spectrum is encountered. Another way of looking at the problem is to consider (4.3) and to analyze the properties of the map of the Fourier coefficients. Since the fixed point $A=0$ is hyperbolic if the frequency of the supposed existing discrete breather fullfills the nonresonance condition (all its multiples are outside the linear spectrum) then the dimension of the stable and unstable manifolds is exactly one half of the phase space dimension of the map. There is a problem in that the phase space dimension is in fact infinite. Still we can find the following. If the DB solution exists, the two invariant manifolds have intersections in common points. For any even space dimension it then follows that such an intersection is either structurally stable against small perturbations of the manifolds, or there exists at least one perturbation such that the intersection becomes structurally stable afterwards [76]. This argument does not depend on the space dimension of the map, so we can consider the limit of infinite dimension afterwards. But changes in the Hamiltonian parameters will indeed only perturb the invariant manifolds. Then we find that discrete breathers are structurally stable provided the nonresonance condition holds [76].

\section{DYNAMICAL STABILITY}

In this section we will discuss the stability of discrete breather solutions with respect to small perturbations in some initial conditions. In contrast to the structural stability we are not changing the Hamiltonian, but slighlty departing in phase space from the DB periodic orbit. We can not in general expect then to stay on a periodic orbit again. The question is then, which properties does the new trajectory have? In particular we will be interested in the question: what are the topological properties of the new trajectory relative to the original DB trajectory? These questions have not been extensively studied. There exist analytical and numerical results of Campbell and Peyrard 41], Marin and Aubry [140], analytical results by Bambusi [16] and numerical results by Flach et al [90], [84]. In the first part of this

\footnotetext{
${ }^{2}$ See also 74 .
} 
section we will discuss the linear stability analysis, which considers the Floquet eigenvalue problem assotiated with the linearized phases space flow around the DB orbit. In this part we will follow closely a paper by Aubry [13]. In the second part we will discuss results on the DB stability in the original system (i.e. without linearizing the phase space flow).

\section{A. Linear stability analysis}

Assume a discrete breather solution to be given (either analytically or numerically): $u_{l}(t)=u_{l}\left(t+T_{b}\right)$. Add a small perturbation $\delta_{l}$ to it (i.e. change say the initial conditions at $t=0$ slightly) and obtain the equations of motion for $\delta_{l}$ using its smallness (i.e. take into account only terms linear in $\delta_{l}$ ):

$$
\ddot{\delta}_{l}=-\frac{\partial^{2} H}{\partial u_{l} \partial u_{m}} \delta_{m} .
$$

This equation describes the linearized phase space flow around the discrete breather solution. Note that it has to contain the subset of perturbations which simply continue the discrete breather solution! Because of the nonlinearity of the problem the right-hand side of (8.1) contains parameters which depend on time. Moreover these parameters are periodic in time with period $T_{b}$ and are spatially localized, so that far away from the breather (8.1) will essentially correspond to the equations of motion of the original system when linearized around the groundstate. This type of equations is a generalization of Hill's equations. It can be associated with keywords like Bloch band theory, Floquet theory, parametric resonance and others, indicating its general applicability to many situations of interest.

The Bloch theorem states that all bounded solutions of (8.1) can be represented in the form

$$
\delta_{l}(t)=\mathrm{e}^{i \omega_{\nu} t} \Delta_{l}^{(\nu)}(t),
$$

where $\Delta_{l}$ is periodic with the period of the parameters in 8.1 i.e.

$$
\Delta_{l}(t)=\Delta_{l}\left(t+T_{b}\right)
$$

The task is then to find the eigenfunctions $\Delta(t)=\left\{\Delta_{l}(t)\right\}$ which are periodic in time and fullfill (8.1) after inserting (8.2). This can be simplified by recognizing that the phase space flow defined by (8.1) carries an initial condition $\delta_{l}$ into $\mathrm{e}^{i \theta_{\nu}} \delta_{l}$ if the $\nu$-th eigenfunction has been excited. Here the Floquet multiplier $\mathrm{e}^{i \theta_{\nu}}=\mathrm{e}^{i \omega_{\nu} T_{b}}$. Then we can consider the Floquet map $F\left(T_{b}\right)$ which maps the phase space $\left\{\delta_{l}, \dot{\delta}_{l}\right\}$ onto itself:

$$
\left\{\delta_{l}\left(t+T_{b}\right), \dot{\delta}_{l}\left(t+T_{b}\right)\right\}=F\left(T_{b}\right)\left\{\delta_{l}(t), \dot{\delta}_{l}(t)\right\} .
$$

This map is symplectic which implies that for any two initial conditions the symplectic product

$$
\sum_{l}\left[\delta_{l}^{(1)}(t) \dot{\delta}_{l}^{(2)}(t)-\delta_{l}^{(2)}(t) \dot{\delta}_{l}^{(1)}(t)\right]
$$

is constant in time [13]. The Floquet map is then a matrix of the rank of the phase space of the problem. The Floquet multipliers are the eigenvalues of this matrix. Those of them which are located on the unit circle $\left(\theta_{\nu}\right.$ is real) correspond to bounded solutions of the original problem (8.1). In general if $\lambda$ is an eigenvalue of $F\left(T_{b}\right)$ so are $1 / \lambda$, $\lambda^{*}$ and $1 / \lambda^{*}$.

The Floquet matrix can be computed numerically. It is in fact nothing but the Newton matrix (which has to operate however on the whole phase space, and not as discussed above on a subset $\left\{\dot{u}_{l}=0\right\}$ ). One has to perform $2 N$ integrations of the equations of motion (8.1), where the input is the time-periodic breather solution itself (it defines the time-dependent parameters). One can use all available symmetries (irreducible representations) like some spatial symmetries assotiated with the DB solution in order to reduce the numerical effort of diagonalization.

Numerically it is quite hard to find that a Floquet multiplier is exactly on the unit circle. Aubry has suggested that the extension of the diagonalization procedure may help both with better interpreting the eigenvalue properties as well as with the analysis of bifurcations - cases when the original DB periodic orbit turns unstable. For that one adds to say the right hand side of (8.1) a linear term $E \delta_{l}$ [13]. Now we can repeat the whole construction and obtain a Floquet matrix parametrically depending on $E$. Solving for each value of $E$ (and of course working in the irreducible representations, i.e. taking into account all symmetries of the matrix) we find a set of bands $E_{\nu}(\theta)$. These bands are of course $2 \pi$-periodic in $\theta$. The original Floquet multipliers are obtained by putting $E=0$. First of all this approach allows for a safer elimination of errors in determining the Floquet multipliers located on the unit circle, because we 
have now a 'correlation' given by $E(\theta)$ (at the expense of more computations). Another important feature of these bands is that they immediately predict the stability/instability of the periodic orbit under investigation. Indeed, the periodic orbit is stable only if all Floquet multipliers are located on the unit circle. Suppose we start with such a case and vary the parameters of the periodic orbit (by e.g. changing the frequency of the DB). Then multipliers can 'collide' and leave the unit circle - either in pairs if the collision happened at \pm 1 or in two conjugated pairs if the collision happened elsewhere on the unit circle. Once such a collision happens, the periodic orbit becomes linearly unstable - because the corresponding perturbations $\delta_{l}$ will exponentially grow in time without limitation. Usually (but not always) new families of periodic orbits (usually stable) bifurcate from the unstable periodic orbit.

However often Floquet multipliers can cross each other on the unit circle without any collision (i.e. they stay after crossing). Clearly from the above a loss of some Floquet multipliers happens whenever a band $E(\theta)$ happens to loose two zeros $E=0$ at $\theta=0$ or $\theta=\pi$ (or four zeroes - two at $\theta_{+} \neq 0$ and two at $\theta_{-}=-\theta_{+}$). Thus Aubry has refined the Krein signature which is a necessary condition for collision to a sufficient condition - namely the behaviour of zeroes of a band $E(\theta)$ [13]. Only Floquet multipliers yielding zeroes of one and the same band can collide and leave the unit circle. From this it also follows, that whenever a multiplier comes close to \pm 1 on the unit cicle, a collision is inavoidable.

There is one consequence of this analysis. Namely it follows immediately that one-site breathers continued from the noninteracting case (antiintegrability limit) are linearly stable - at least up to some finite interaction strength [13].

Given the above it is a matter of numerical calculation to analyze the linear stability of discrete breathers. Marin and Aubry [140] show discrete breathers can be linearly stable or unstable and all of the above discussed collisions can be observed.

Because we usually do not know the analytical form of the DB solution and because we study a lattice, it is hard to predict analytically the stability behaviour of DBs. Indeed the Floquet matrix diagonalization yields both extended and localized eigenvectors. The existence of localized eigenvectors has been long suggested in the literature (stability/instability of even/odd parity modes [157], [46], XC/XN modes [51], [129]). In fact in many cases the spatial symmetries of DB solutions have been correlated with the dynamical linear stability. This aspect deserves more clarification. Especially it seems plausible that the XC discrete breather - which is just an one-site breather in the antiintegrable limit - has to be stable at least for weak interactions. This result has been also found in the numerical analysis of Campbell and Peyrard [41]. There for some parameter ranges the XN solution (a two-site breather) was found to be linearly unstable, whereas the XC solution was stable. We will discuss these aspects in section $\mathrm{X}$ when discussing band edge plane wave bifurcations.

Suppose we solve the linear stability problem for an infinite system. All extended Floquet eigenvectors will then have the form of linear plane waves far from the breather center. Consequently we know that all extended eigenvectors have $\theta=\omega_{q} T_{b}=\omega_{q} / \omega_{b} 2 \pi$. Because the DB is exponentially localized, it can cause only a finite number of Floquet eigenvectors to be localized. Consequently there will be an infinite number of extended eigenvectors, whose eigenvalues will densely fill the linear spectrum. We can immediately derive some dangerous DB frequencies causing instabilities (this result was derived by Flach and Willis in another way [87]):

$$
\omega_{q} / \omega_{b}=k / 2 \quad, \quad k=1,2,3, \ldots .
$$

Even $k$ correspond to collisions of Floquet multipliers at +1 , and odd $k$ at -1 . The cases $k$ even correspond to the resonance conditions when discrete breathers stop existing (section IV). In fact we can expect that for $k=2$ the breather disappears, but for larger even values of $k$ the breather becomes a nanopteron 34 - i.e. a localized object with nondecaying tails. Consequently nanopterons are dynamically unstable (if they exist at all)! Indeed Marin and Aubry report about these nanopterons in numerical calculations [140]. The cases $k$ odd correspond to period doubling bifurcations. The breather can still exist but will be dynamically unstable. The relevant perturbations however will be extended, so that the new periodic orbits bifurcating from the old one must again have the spatial shape of nanopterons!

\section{B. Going beyond linearization}

What happens to the solutions $\delta_{l}$ if we do not impose the linearization (8.1) but instead solve the original equations of motion? One answer has been recently found by Bambusi [16]. He showed that periodic breather orbits are exponentially stable in the antiintegrable limit. That means that after a suitable chosen metric has been introduced, the perturbation of a periodic DB orbit stays close to the orbit up to exponentially large times. Let us be more precise: if the distance $d$ of the perturbed orbit from the periodic one is smaller than a value proportional to the square root of the interaction strength, then the distance stays close to the periodic orbit for times proportional to the exponential of some inverse power of the interaction (times a constant). The frequency of the DB in the antiintegrable limit (zero 
interaction) has to be not only in nonresonance with the degenerate linear spectrum, but it has to be diophantine with the linear spectrum. Details can be found in [16]. Further Bambusi shows that a 'quasiperiodic' breather object although not existing as a precise solution can be a good approximation to a real trajectory for times again exponentially large (in the same meaning as above). The small errors eventually acumulating with time are referred to as radiation.

This radiation has been studied numerically. Indeed the numerical experiments for long-lived localized excitations discussed in section III in fact study perturbed discrete breather solutions. What can we learn from those results? First, as long as the object is not a periodic DB, it radiates energy in the form of small amplitude plane waves exactly as predicted in section [V] and obtained by Bambusi. This decay can be very weak - in fact so weak that it is hard to measure! Estimates of the decay come up with a loss of 'breather' energy per 'period' of oscillation (note that we are not dealing with a strict time-periodic solution) of the order of $0.01 \%-0.001 \%$ in units of the 'breather' energy [90]! From this circumstance it follows that even though discrete breathers form a subset of measure zero in the phase space of nonlinear lattice, their phase space support can be finite and in fact the lifetimes of objects associated with DB solutions can be tremendously large, making the breather concept highly reasonable.

Another interesting question is - what happens to a perturbed breather if we wait long enough? Will the object radiate its whole energy away and vanish or not? In Fig.9 we show the dependence of the object's energy on time for different initial conditions (for details see [9]]). The internal timescale is of the order of 10. As long as the radiation is weak, we can view the object as a quasiperiodic breather whose parameters (energy, frequencies) are slowly timedependent. Then we can characterize the object by those parameters which are slowly varying functions. Several results have been observed. For some initial perturbations the object radiates away energy and consequently slowly approaches the family of periodic breather orbits. Clearly the radiation becomes weaker and weaker as the periodic orbit family is approached. This behavior reminds us of an attractor in a dissipative system. In fact because the radiation becomes exponentially weak, on finite computing time scales we even observed a seemingly forever living quasiperiodic breather. To see that we plot in Fig.10 a Poincare map of a perturbed DB of a two-dimensional lattice, where consecutive points are connected with straight lines. The attractor-like behaviour is seen, together with a seemingly remaining nonzero distance from the fixed point (periodic orbit) in the middle of the figure (for details see [84]).

For some trajectories we find that internal resonances between the internal frequencies describing the quasiperiodic evolution on short time scales cause an 'explosion' of the breather-like object (see Fig.9 and [90]), which in a local analysis was due to a chaotic dynamics inside the breather - remarkably that does not cause the whole structure to disappear! Moreover, after some radiation which is still weak, of the order of $1 \%$ of the breather energy per breather period, the radiation suddenly stopped at energies 0.35 . A local phase space analysis yielded that the strong chaotic trajectories disappear around these energies. Consequently we find that perturbed discrete breathers can slowly radiate energy away and thus are either attracted to the periodic orbit family (consequently stabilized) or repelled. In the second case internal resonances can trigger chaotic local dynamics which causes an increase of the radiation rate by two orders of magnitude. Still even those cases eventually end up with a breather-like object which is again weakly decaying, most probably into an exact time-periodic breather.

This indicates that the linearized stability analysis can be of help but does not tell important things - because it is likely that all the evolutions of breathers discussed here were initiated by perturbing linearly stable discrete breathers!

\section{MOVABILITY}

So far we have been discussing discrete breather solutions which are time periodic. By definition these solutions are stationary, i.e. they do not 'move' through the lattice. We have been also discussing small perturbations of DB solutions, which were supposed to stay small (at least for several DB periods). Again such trajectories do not correspond to a 'moving' entity. However we know that e.g. for the sG PDE the breather solution can be Lorentzboosted, due to the invariance of the sG PDE under Lorentz transformations. Such a boosted breather would actually

move, i.e. its center is propagating with constant velocity. That is certainly not a time-periodic solution anymore. If we consider a lattice we loose the continuous symmetry in space and replace it with a discrete symmetry. What can be said about the existence of objects similar to boosted sG breathers in lattices?

\section{A. Numerical findings}

A good way to start with are numerical simulations of lattices. Takeno et al (see e.g. 107, 108, [174, [171, [173]) indeed have reported on moving breathers in one-dimensional FPU lattices. Apparently the breathers are very 
localized in space and move over long distances without significantly radiating energy or changing their shape (which would be a sign that the object does not correspond to a moving breather). Note that the internal frequencies are above the linear spectrum, and the observed velocities are below the maximum group velocity of linear plane waves. A careful numerical study relating dynamically unstable DBs with moving breather-like objects has been performed by Sandusky, Page and Schmidt [157]. Sievers and Page summarized numerical results on moving breathers in [162].

A different picture emerges for one-dimensional Klein-Gordon lattices. There moving breather-like objects are typically only found if the amplitude of the object is small enough - which in turn implies that these objects are only weakly localized in space. If the amplitude becomes larger (and the objects stronger localized) then typically one observes radiation and a subsequent stopping of the object. Once it stops the object is similar to a perturbed DB (see Bang and Peyrard [17]).

Yet another result comes if we consider two-dimensional lattices. While nothing drastically changes for KleinGordon lattices 179, the two-dimensional FPU lattice resists in producing moving breather-like objects which are strongly localized - as opposed to the corresponding one-dimensional case 36.

So the emerging picture is quite inhomogeneous. Moving breather-like objects are detected whenever the amplitudes are fairly small and the objects are weakly localized, and for some systems (1d FPU lattices) even for large amplitudes (objects are strongly localized). Moving objects are not detected whenever the amplitudes are large and the localization is strong (for Klein-Gordon lattices), and for lattice dimensions larger than one (for FPU lattices). Note that even when we say that moving breathers are detected that does not imply that there exist lossless exact solutions - it just means that during the time of the numerical experiment the object did not radiate an appreciable amount of energy away ( typically the threshold is $1 \%-5 \%$ ).

One emerging pattern seems to be that small-amplitude breathers can move - at least over much larger times (and distances) than large-amplitude breathers. There is a natural explanation of this observation. If the breather-like object is weakly localized, its envelope is slowly varying in space. As discussed in section II, Kosevich and Kovalev used this circumstance to derive in some lowest order a partial differential equation! Even though this is not exact, it certainly can describe a real trajectory over an increasingly larger time window with decreasing amplitude of the object. But since the corresponding PDE possesses a continuous symmetry (in space) its stationary solutions can be boosted, such that they move! So moving breather-like objects appear to be quite reasonable for weakly localized small-amplitude objects. Most probably they will not move forever because we are actually solving a lattice problem (unless there is some other symmetry involved). But in the limit of small amplitudes the time scale over which the objects appreciably change (decay) might diverge, thus escaping from any finite-time window in a numerical experiment.

Of course there is still the possibility that exact moving breather solutions exist! And they do exist - for the Ablowitz-Ladik lattice, which is integrable [1] 3. However neither do there exist existence proofs for other lattices, nor are the numerical results conclusive enough to support the existence of moving breather in nonintegrable lattices. The situation is clearly different from the circumstances of finding stationary discrete breathers, where numerical investigations strongly suggested that time-periodic DBs exist independent on the integrability properties of the lattice.

\section{B. What can we learn from moving lattice kinks?}

Before asking whether moving breather solutions exist it is useful to see what is known about moving kinks in lattices. Recall that a kink solution is defined by some nontrivial boundary condition $\left(u_{l \rightarrow+\infty}-u_{l \rightarrow-\infty}\right)=\eta \neq 0$. If a Klein-Gordon PDE supports static kink solutions, it again supports boosted (moving) kink solutions due to the continuum symmetry. Things are different for lattices. First of all there exist exact moving kinks in the Toda lattice - which is however integrable. McLeod [142] has given an analytical proof of continuation of moving Toda kinks into other one-dimensional FPU systems. Duncan et al [59] have verified this result numerically up to machine precision. At the same time MacLoyd was unable to continue moving kinks into Klein-Gordon one-dimensional lattices, and the numerical results of Eilbeck et al suggest that moving kinks do not exist in a sG lattice - instead an object similar to the nanopteron was found - namely a kink-like structure which becomes a plane wave in the tails.

For Klein-Gordon chains where exact moving kinks seem not to exist, kinks carry a topological charge [58]. This implies esentially that in order to remove one kink, one needs to overcome an infinitely high energy barrier (for an

\footnotetext{
${ }^{3}$ Fischer 75] considered a three-particle FPU system which is integrable. Solutions similar to stationary discrete breathers and moving breathers do exist there. However nothing similar is known for the case of an infinite lattice.
} 
infinite system). So even though a moving kink does not exist, the kink can not disappear (note that there is no topological charge associated with moving FPU kinks). Thus a Klein-Gordon kink when boosted can only radiate some energy away and finally stop to become a static kink solution! In order to account for these effects a collective coordinate approach was developed [195], [165], [28], 194], [29], 193], [57], [30]. Within this approach one performs a canonical transformation to new coordinates some of whom are collective (nonlocal in the old coordinates). These collective coordinates describe the kink. As a result one can obtain equations of motion of the kink, which are of course coupled to the infinite number of the remaining degrees of freedom. Using some constraints imposed on the kink's position one can evaluate the energy of a static kink at different positions. This energy will be periodic with the period of the lattice, and in general be not constant. Consequently it is labeled Peierls-Nabarro potential, because in the limit of a nearly static kink it appears as a potential in the nearly Newtonian equations of motion for the kink. The potential will have at least one minimum and maximum between two lattice sites. These extrema define the true static kink solutions on the lattice, and the difference of the heights of the extrema is called Peierls-Nabarro barrier $E_{P N}$. The kink corresponding to the maximum of the Peierls-Nabarro potential is unstable with respect to small perturbations (it corresponds to a saddle point in the potential energy relief of the original Klein-Gordon system), and the kink corresponding to the minimum of Peierls-Nabarro potential is stable with respect to small perturbations (it corresponds to a local minimum in the potential energy relief of the original Klein-Gordon system). It is argued that exactly the energy $E_{P N}$ is needed by the stable kink solution in order to overcome the lattice pinning and to move. In turn if a moving kink is losing energy by radiating plane waves, it should be trapped by the lattice at some moment in time.

This concept has been proven to be very successful. Qualitatively its predictions indeed take place. Quantitatively we have to take into account the kink interaction with the plane waves, in order to obtain radiation. This can be still done up to some degree analytically, and we think it is possible to say that the Peierls-Nabarro concept is an effective way to reduce the problem of handling the infinite-dimensional phase space flow to a finite-dimensional one. Note that the concept works however only in systems which allow for static kinks - nothing similar has been done e.g. for FPU chains lacking static kink solutions but allowing for exact moving kinks!

The success of this approach is based on the fact that we can reduce the problem of the Peierls-Nabarro barrier to the energy difference of certain extrema of a potential function. Indeed, if we consider a local minimum of such a function, then we need to find the lowest-lying saddle in order to escape. No matter what we do, if the additional energy supply to the local minimum state is lower than the difference to the lowest lying saddle, we can not escape! So the $E_{P N}$-value becomes a meaningful depinning energy which can be used e.g. in a statistical analysis.

\section{The movability separatrix}

It is tempting to use the approach for lattice kinks as a description of moving breathers. The ultimative goal would be the calculation of a depinning energy. Indeed some numerical indications for discrete breathers are quite reasonable in that context - there exist seemingly always stable and unstable stationary DB solutions, and moving breather-like objects radiate energy and eventually stop (are pinned by the lattice) etc [52], 117, [18].

Such a task has been performed in [47], [129]. Discrete breather solutions were parametrized (internal frequency and position on the lattice). A projection of the phase space flow onto a low-dimensional subspace yielded indeed energy values which have been coined Peierls-Nabarro energies, in analogy to moving kinks. In the following we will have to extend the notion of a Peierls-Nabarro energy to a movability separatrix, which does not allow the definition of a depinning energy in general. There is no depinning energy for discrete breathers.

First of all we mention the numerical analysis of Bang and Peyrard [17]. They have analysed the movability properties of discrete breathers. By considering a multiple-scale expansion for the equations of motion up to third order (which is only useful for small amplitudes) moving breather solutions were obtained. Of course these solutions are approximative, so Bang and Peyrard used these approximative solutions as initial conditions for the true equations of motion. Their very extensive analysis showed that the projections of the phase space flow done in 47 yielded quantitative discrepances of orders of magnitude.

Let us now immediately answer the question: why is it impossible to introduce a depinning energy? The answer is: simply because the stationary discrete breather solutions we want to depin are not isolated - i.e. they come in one-parameter families. Suppose that we have a certain solution of this family of solutions which at some time $t=0$ is given by a certain point $P_{1}$ in the phase space of the system. The energy of this point is $E\left(P_{1}\right)$. Now assume we can define a depinning energy which has to be added to this solution in order to depin it from the lattice (to let it move). That means that we can add a certain perturbation of the initial conditions of the discrete breather such that the new point $P_{2}$ in phase space will have a higher energy $E\left(P_{2}\right)>E\left(P_{1}\right)$ and that the trajectory which is defined by $P_{2}$ corresponds to some moving breather-like object. Then we could always choose another stationary discrete 
breather solution on the original one-parameter family with a different energy. Since there exist no upper bounds on energies of discrete breathers in general, this energy can be larger than $E\left(P_{2}\right)$. Adding to this second discrete breather a perturbation which brings it right back to $P_{2}$ we thus can depin a discrete breather by adding negative energy! In fact it is clear that we can find any situation, in other words we are able to add or substract energy from a discrete breather and by choosing the right perturbation depin it! Certainly this makes clear that the concept of depinning energies does not make the least sense when applied to discrete breathers.

In order to understand more we can follow a phenomenological approach which has been successfully tested in numerical experiments [88] To describe a periodic elliptic (linearly stable) DB we need to introduce one degree of freedom, which describes the one-parameter set of DB solutions. We will work in the action-angle phase $(J, \theta)$ space and name this degree of freedom $J_{1}$. Its corresponding frequency will be $\omega_{1}=\dot{\theta}_{1}=\partial H / \partial J_{1}$. Here $H$ denotes the full Hamiltonian of the lattice. We assume that there exists a certain transformation between the original variables (positions, momenta) and the actions and angles. This does not imply integrability of the system as well as it does not imply the inverse. Since the DB solutions are regular solutions (at least on moderate time scales) there is no need in introducing stochasticity (cf. 90] for details). We assume that the DB solutions have a spatial symmetry. To excite a moving NLE we have to excite an additional degree of freedom $J_{3}$. Exciting $J_{3}$ we destroy the spatial symmetry of the linearly stable DB. But since it is always possible to perturb the DB conserving the symmetry, we have to include an additional symmetryconserving degree of freedom $J_{2}$ into the consideration. Thus we end up with the simplest generic case of a Hamiltonian problem with three degrees of freedom:

$$
H=H\left(J_{1} ; J_{2} ; J_{3}\right) \quad, \quad \omega_{i}=\dot{\theta}_{i}=\frac{\partial H}{\partial J_{i}}, \quad i=1,2,3 .
$$

According to our notation $i=3$ labels the symmetrybreaking degree of freedom. If it is excited strongly enough we expect to hit a separatrix which separates stationary DBs from moving ones. We will name this separatrix movability separatrix. All three degrees of freedom are assumed to be of local character, especially they can be well defined in the reduced problem for the DB.

Let us state the general condition for the movability separatrix we are looking for. Since on the movability separatrix a trajectory will for infinite times asymptotically reach a hyperbolic state (which is a linearly unstable DB manifold and its space-symmetric perturbations) the corresponding frequency of the $3 \mathrm{~d}$ degree of freedom

$$
\omega_{3}=\frac{\partial H}{\partial J_{3}}=f\left(J_{1} ; J_{2} ; J_{3}\right)
$$

has to vanish on the movability separatrix i.e.,

$$
f\left(J_{1} ; J_{2} ; J_{3}\right)=0,
$$

which implies an equation for a surface in the three-dimensional subspace of the actions $\left(J_{1} ; J_{2} ; J_{3}\right)$. We can always eliminate $J_{2}$ using the expression for the energy $E=H\left(J_{1} ; J_{2} ; J_{3}\right)$, so that (9.3) yields:

$$
f\left(J_{1} ; J_{2} ; J_{3}\right)=\tilde{f}\left(E ; J_{1} ; J_{3}\right)=0 .
$$

From (9.4) it follows that there exists a critical value for $J_{3}$ on the movability separatrix:

$$
J_{3}^{s}=g\left(J_{1} ; J_{2}\right)=\tilde{g}\left(E ; J_{1}\right) \quad .
$$

The critical value $J_{3}^{s}$ depends both on $E$ and $J_{1}$. Only for nongeneric cases when $\tilde{g}$ is constant would we be able to introduce an extended notion of a depinning energy by considering a subspace of relevant perturbations. However as shown in 88 this is not possible in general, and thus we have to accept that stationary linearly stable discrete breathers, which come in one-parameter families and thus form two-dimensional manifolds in phase space, are separated by a movability separatrix from possible moving solutions. The concept of a depinning energy therefore has no meaning.

The existence of a movability separatrix implies that if we consider a trajectory which corresponds to breather-like moving object (at least over some period of time) then the presence of the separatrix will show up in a modulation of the time-dependence of the object if parametrized in some meaningful way. This modulation is due to the presence of the frequency $\omega_{3}$ from above. It follows that this frequency must be related to the periodicity of the lattice through the motion of the object. However we measure the averaged propagation of the object $v$ where distance is measured in units of lattice spacing - it will be thus related to $\omega_{3}$ through the relation $\omega_{3}=2 \pi v$. Sandusky, Page and Schmidt have observed precisely this relation by independently measuring $v$ and $\omega_{3}$ in careful numerical experiments (Figs. 7 and 8 in [157). 


\section{Breather-breather collisions}

In a series of numerical experiments Peyrard et al [52], [18], [93] have analyzed the collisions between discrete breathers. Typically moving breather-like objects were excited and the effects of many collisions studied. Because of our lack of understanding of moving solutions the approach to describe the observed phenomena in collisions is heuristic. It has been observed that the breathers survive collisions, but exchange energy. The energy exchange seems to depend on some phase differences (note that we have to introduce a parametrization of moving breathers, a rather delicate task). Eventually breathers which collect enough energy become too localized in space and trap on the lattice, stopping their energy collection. This problem needs further clarification.

\section{E. Do moving breathers exist?}

We have still no answer to the question whether moving breathers exist. By requiring that after some traveling time a breather is exactly recovered at some other location in the lattice we would have to solve for differential equations with advanced and retarded terms. Moving breathers would correspond to certain homoclinic orbits of this equation. We have no knowledge about solutions which correspond to moving discrete breathers.

\section{PLANE WAVE BIFURCATIONS AND DISCRETE BREATHERS}

So far we did not pose the question: how are discrete breathers connected to the plane wave solutions of the linearized equations of motion? In fact there exists a well-known approach in the literature which is coined modulational instability. Originally this appoach was designed for the study of waves in continuous media - some older papers use also the term Benjamin-Feir instability instead [196]. Within this approach a plane wave solution of the linearized equations of motion is continued into the weakly nonlinear regime. Small plane wave perturbations (of different wave length) are then added and the stability of the perturbed wave is analyzed. This approach has been very helpful in connecting the instability of certain modulated plane waves with spatially localized solutions, which exist because the nonlinearity of the system effectively prevents a dispersing of the object. Thus the stability study of plane waves can become crucial when predicting the existence of localized solutions without actually calculating the latter.

Modulational instability has been analyzed for lattices with respect to discrete breathers in a number of publications by Kivshar and Peyrard [125], Flytzanis, Pnevmatikos and Remoissenet [92], Tsurui [184] and Sanduski and Page [156.

Here we will follow the approach described in [8], where the stability analysis was performed for finite systems. Strictly speaking we have to analyze the stability properties of a periodic orbit (plane wave). For that we have to linearize the phase space flow around the periodic orbit - just as we did for the discrete breather. Then we have to find the eigenmodes and eigenfrequencies. Thus it is reasonable to perform a stability analysis of plane waves for a large but finite system. This will then lead to results which depend on the size of the system. The stability dependence on the size of the system is crucial when understanding properties of discrete breathers in different lattice dimensions (cf. section XI)! Moreover in this case we can connect the instability of a plane wave with a bifurcation of new periodic orbits - a connection hard to make for the infinite system approach. Further we will be able to prove that the new bifurcating periodic orbits can not be invariant under discrete translations along the lattice - as expected for discrete breather solutions!

First we will argue why we have to consider band edge plane waves when we want to understand the connection to discrete breathers. Secondly we will review the plane wave stability analysis for finite systems and discuss its consequences for discrete breathers. Finally we will make some predictions.

\section{A. Why the band edge plane wave?}

If discrete breathers are connected to plane waves, we have to look for breather solutions which are weakly localized on the lattice. This seems to happen only when the frequency of the discrete breather is close to a band edge of the linear spectrum. That in turn implies that the amplitude of the breather center is small. Consequently it appears to be logical to expect that discrete breather periodic orbits which belong to a family of solutions which contains weakly localized small-amplitude DBs appear through bifurcations of band edge plane waves, i.e. plane wave periodic orbits which in the limit of small amplitude correspond to normal modes with frequencies at the edge of the linear spectrum. Recall that there can be several band edges of the linear spectrum in principle. This follows from the fact 
that regardless from what periodic orbits DBs bifurcate, once the frequency of the DB is close to the band edge of the linear spectrum, the DB becomes by definition a weakly modulated band edge plane wave, in other words its internal spatial symmetry will be very close to the spatial symmetry (defined by the corresponding wave vector) of the band edge plane wave. Consequently we can rule out, that a DB family might bifurcate from any other plane wave but the band edge plane wave. But then it follows that the frequency (period) of the DB close to the bifurcation is very close to the frequency (period) of the band edge plane wave, i.e. the bifurcation is expected to be a tangent one! By that we mean that the Floquet multipliers of the linearized stability analysis of the band edge plane wave have to collide at +1 on the unit circle! With these statements in mind we will then perform the Floquet analysis of the band edge plane wave.

Before we proceed we mention another possibility of DB occurence which has not yet been studied. The concept of the nanopteron (a breather-like object with some resonances of multiples of its frequency with the linear spectrum) allows for cases when DBs occur through analytical continuation of nanopterons. Consider a linear spectrum which has two gaps at least. Number the gaps 1,2 with increasing frequency values. Then a breather can exist with $\Omega_{b}$ in gap 1, a second harmonic in gap 2, and the rest of the harmonics above the linear spectrum. It is also possible to choose the linear spectrum such, that either lowering or increasing $\Omega_{b}$ will lead to a resonance of $2 \Omega_{b}$ with the linear spectrum first. When lowering $\Omega_{b}$ the second harmonic $2 \Omega_{b}$ touches the upper band edge of the linear spectrum located between gaps 1 and 2, and increasing $\Omega_{b}$ yields a first touch of $2 \Omega_{b}$ with the lower band edge of the linear spectrum band located above gap 2. Then in both cases we can expect the DB to become a nanopteron. Consequently this DB family will never make contact with a plane wave. Thus for complicated linear spectra the existence of some families of DB solutions might be not connected to the stability of band edge plane waves at all!

\section{B. Tangent bifurcations of band edge plane waves}

Consider system (3.1) for a finite size $N$ of the lattice. We assume periodic boundary conditions

$$
u_{l}=u_{l+N} \quad, \quad \dot{u}_{l}=\dot{u}_{l+N} .
$$

Then system (3.1) exhibits permutational symmetry. The permutational operator $\hat{P}$ is defined by

$$
\hat{P} g\left(u_{1}, u_{2}, \ldots, u_{N}, \dot{u}_{1}, \dot{u}_{2}, \ldots, \dot{u}_{N}\right)=g\left(u_{2}, u_{3}, \ldots, u_{N}, u_{1}, \dot{u}_{2}, \dot{u}_{3}, \ldots, \dot{u}_{N}, \dot{u}_{1}\right) .
$$

Clearly $\hat{P}^{N}=\hat{1}$ and $\hat{P} H=H$.

Let us introduce normal coordinates

$$
Q_{q}=\frac{1}{N} \sum_{l=1}^{N} \mathrm{e}^{i q l} u_{l} .
$$

The wave number $q$ can take any of the values

$$
q=\frac{2 \pi}{N} n, \quad n=0,1,2, \ldots,(N-1) .
$$

The inverse transform of (10.2) is given by

$$
u_{l}=\sum_{q} \mathrm{e}^{-i q l} Q_{q}
$$

The equations of motion for the normal coordinates $Q_{q}$ read

$$
\ddot{Q}_{q}=\frac{1}{N} \sum_{l=1}^{N} \mathrm{e}^{i q l} \ddot{u}_{l}=-\frac{1}{N} \sum_{l=1}^{N} \mathrm{e}^{i q l} \frac{\partial H}{\partial u_{l}} .
$$

Using (3.1) and (3.2), (3.3) we obtain the following lengthy expression

$$
\begin{aligned}
& \ddot{Q}_{q}=-\Omega_{q}^{2} Q_{q}-\sum_{\mu=3}^{\infty} v_{\mu} \sum_{q_{1}, q_{2}, \ldots, q_{\mu-2}}\left[\prod_{\nu=1}^{\mu-2} Q_{q_{\nu}}\right] Q_{q-\sum_{\nu=1}^{\mu-2} q_{\nu}}- \\
& -\sum_{l=1}^{N} \mathrm{e}^{i q l} \sum_{\mu=3}^{\infty} \phi_{\mu}\left[\left\{\sum_{q^{\prime}}\left(1-\mathrm{e}^{i q^{\prime}}\right) \mathrm{e}^{-i q^{\prime} l} Q_{q^{\prime}}\right\}^{\mu-1}-\left\{\sum_{q^{\prime \prime}}\left(\mathrm{e}^{-i q^{\prime \prime}}-1\right) \mathrm{e}^{-i q^{\prime \prime} l} Q_{q^{\prime \prime}}\right\}^{\mu-1}\right] .
\end{aligned}
$$


Here $\Omega_{q}$ abbrevates the eigenfrequencies of the linearized (in $Q_{q}$ ) equations of motion and is given by the dispersion relation

$$
\Omega_{q}^{2}=v_{2}+4 \phi_{2} \sin ^{2}\left(\frac{q}{2}\right)
$$

Let us give the solutions for two periodic orbits of the considered lattice, which correspond to the band edge plane waves $\left(q=0\right.$ and $\left.q_{N / 2}=\pi\right)$ in the limit of small energies:

$$
\begin{array}{r}
\mathrm{I}: Q_{q \neq 0}=0, \ddot{Q}_{q=0}=-\sum_{\mu=2}^{\infty} v_{\mu} Q_{q=0}^{\mu-1}, \\
\mathrm{II}: Q_{q \neq \pi}=0, \quad \ddot{Q}_{q_{N / 2}}=-\sum_{\mu=2,4,6, \ldots}^{\infty} \bar{v}_{\mu} Q_{q_{N / 2}}^{\mu-1} .
\end{array}
$$

The paramter $\bar{v}_{\mu}$ is given by

$$
\bar{v}_{\mu}=v_{\mu}+2^{\mu} \phi_{\mu}
$$

In case II we have to demand

$$
\text { II : } v_{2 m+1}=0
$$

in order to be able to continue the upper band edge plane wave to finite energies in the given form. The terms $\phi_{2 m+1}$ can be in general nonzero, but they simply do not contribute to (10.8) because of the odd symmetry of the upper band edge plane wave 156].

\section{Tangent bifurcation of orbit I}

Let us consider a small perturbation $\left\{\delta_{q}\right\}$ of the periodic orbit I

$$
Q_{q} \rightarrow Q_{q}+\delta_{q}
$$

Linearizing the equations of motion for the perturbation we obtain

$$
\ddot{\delta}_{q}=-\omega_{q}^{2} \delta_{q}-\sum_{\mu=3}^{\infty}(\mu-1) v_{\mu} Q_{q=0}^{\mu-2} \delta_{q} .
$$

For $q=0$ equation (10.9) describes the continuation of the periodic orbit itself. All other perturbations do not couple with each other, so that we can consider (10.9) for each value of $q$ separately. If we increase the energy of the periodic orbit

$$
E_{I}=\frac{1}{2} \dot{Q}_{q=0}^{2}+V\left(Q_{q=0}\right)
$$

then the first tangent bifurcation will occur if $q=q_{1}=2 \pi / N$ is choosen in equation (10.9). This is so because the associated linear mode frequency is the closest to the band edge frequency. Here the tangent bifurcation implies a collision of two Floquet multipliers at +1 . The accounting of the bifurcation is nothing but a calculation of Arnold tongues in the theory of parametric resonance [11]. The explicit calculation can be done in perturbation theory. Perturbation theory can be applied only if the resulting bifurcation amplitudes or related variables $\left(E_{I}\right)$ are small. The reader will find all details in [80]. For large values of $N$ we have

$$
\Omega_{q_{1}}^{2}=v_{2}+4 \phi_{2} \frac{\pi^{2}}{N^{2}}
$$

and consequently obtain (see [80] for a detailed evaluation) the following bifurcation energy $E_{I}^{c}$ :

$$
\begin{aligned}
& \text { i) }: \phi_{2}=0, E_{I}^{c} \text { arbitrary }, \\
& \text { ii) : } E_{I}^{c}=\frac{1}{N^{2}} \frac{12 \pi^{2} v_{2}^{2} \phi_{2}}{10 v_{3}^{2}-9 v_{2} v_{4}} .
\end{aligned}
$$


Solution ii) is correct for large system sizes, because in the limit of large $N$ the critical amplitude of the band edge plane wave is inverse proportional to $N$, and thus the application of perturbation theory is justified. Since we have to require positive values for $E_{I}^{c}$ and $\phi_{2}$ tangent bifurcation can take place only if

$$
\frac{v_{4}}{v_{2}} \leq \frac{10}{9} \frac{v_{3}^{2}}{v_{2}^{2}}
$$

Condition (10.13) is equivalent to the condition, that the frequency of the lower band edge plane wave decreases with increasing energy. In other words, the sufficient condition for a tangent bifurcation of the lower band edge plane wave is the repelling of its frequency from the linear spectrum $(10.6)$ with increasing energy.

The energy $E_{I}$ is on the scale of total energy per particle (cf. (3.1), (10.2), (10.10)). Consequently the amplitude threshold of the tangent bifurcation decreases as $N^{-1}$ for the individual $u_{l}$ amplitudes. In the limit $N \rightarrow \infty$ the threshold goes to zero.

\section{Tangent bifurcation for orbit II}

We again derive the linearized equations for the perturbation $\delta_{q}$ :

$$
\begin{array}{r}
\ddot{\delta}_{q_{a, b}}=-\omega_{q_{a, b}}^{2} \delta_{q_{a, b}} \sum_{\mu=4,6, \ldots}^{\infty}(\mu-1) \overline{\bar{v}}_{\mu, q_{a, b}} Q_{q_{N / 2}}^{\mu-2} \delta_{q_{a, b}} \\
-i \sum_{\mu=3,5, \ldots}^{\infty}(\mu-1) \overline{\bar{\phi}}_{\mu, q_{b, a}} Q_{q_{N / 2}}^{\mu-2} \delta_{q_{b, a}} .
\end{array}
$$

Here we have used the following notations:

$$
\begin{gathered}
\overline{\bar{v}}_{\mu, q_{a, b}}=v_{\mu}+\sin ^{2}\left(\frac{q_{a, b}}{2}\right) 2^{\mu} \phi_{\mu}, \\
\overline{\bar{\phi}}_{\mu, q_{a, b}}=-\sin \left(q_{a, b}\right) 2^{\mu} \phi_{\mu} .
\end{gathered}
$$

The two wave numbers $q_{a, b}$ are related to each other by

$$
q_{b}=q_{a} \pm \pi \bmod 2 \pi .
$$

In contrast to the previous case we have a coupling between a pair of normal coordinates (10.17) in (10.14). Notice that the coupling term is given by the second sum on the right hand side of (10.14) and is zero if $\phi_{2 m+1}=0$. Also this coupling term is proportional to $i$, which causes a mixing of real and imaginary parts of the perturbations. Interestingly for the pair $q_{a}=\pi, q_{b}=0$ we obtain again no coupling, because (10.16) vanishes for both wavenumbers. Consequently for $q=\pi$ (10.14) describes the continuation of the periodic orbit II.

\section{The case $\phi_{2 m+1}=0$}

If we assume $\phi_{2 m+1}=0$ then the equations for the perturbations $\delta_{q_{a, b}}$ decouple. In analogy to the case of orbit I we have the first tangent bifurcation of orbit II if we consider the perturbation $q=\pi(1-2 / N)$. Consequently we obtain for the bifurcation energy $E_{I I}^{c}$

$$
\begin{array}{r}
\text { i) }: \phi_{2}=0, E_{I I}^{c} \text { arbitrary }, \\
\text { ii) : } E_{I I}^{c}=\frac{1}{N^{2}} \frac{v_{2}+4 \phi_{2}}{3\left(v_{4}+16 \phi_{4}\right)} 4 \pi^{2} \phi_{2} .
\end{array}
$$

Again we observe that a bifurcation will take place only if $\bar{v}_{\mu}$ is positive, i.e. only if the frequency of the periodic orbit II is repelled from the linear spectrum with increasing energy. 
Now the coupling between two perturbations has to be taken into account. One could expect that in the limit of large $N$ the coupling between $q_{a}$ and $q_{b}$ vanishes. That is indeed so if we require $v_{2} \neq 0$ (linear spectrum is optical-like) but turns out to be wrong for the case $v_{2}=0$ (linear spectrum is acoustic-like). The details of these subtleties are given in 80 . Here we proceed to the final result for the tangent bifurcation energy $E_{I I}^{c}$ :

$$
\begin{aligned}
& \text { i) }: E_{I I}^{c}=\frac{1}{2 \phi_{3}^{2}}\left(v_{2}+4 \phi_{2}\right)\left(\phi_{2}+\frac{3}{16} v_{2}\right) \phi_{2} \\
& \text { ii) }: E_{I I}^{c}= \begin{cases}\frac{4 \pi^{2}}{N^{2}} \frac{v_{2}+4 \phi_{2}}{3\left(v_{4}+16 \phi_{4}\right)} \phi_{2} & v_{2} \neq 0 \\
\frac{16 \pi^{2}}{N^{2}} \frac{\phi_{2}^{3}}{3 \phi_{2}\left(v_{4}+16 \phi_{4}\right)-64 \phi_{3}^{2}} & v_{2}=0\end{cases}
\end{aligned}
$$

As we can see solution i) $(10.20)$ is always positive (since $\phi_{2}>0$ and $v_{2} \geq 0$ ) but is not dependent on $N$. Since we applied perturbation theory, (10.20) is correct only in the limit of small energies. For larger energies corrections apply. Solution ii) (10.21) is the one which gives arbitrarily small bifurcation energies for sufficiently large $N$. Since $\phi_{3}$ does not enter the energy dependence of periodic orbit II, we then again obtain as the necessary condition for the existence of the bifurcation, that the frequency of the upper band edge plane wave has to be repelled from the linear spectrum with increasing energy. For the optical-like spectrum case $v_{2} \neq 0$ this is again a sufficient condition. However for the case $v_{2}=0$ a more restrictive condition is obtained by demanding

$$
3 \phi_{2}\left(v_{4}+16 \phi_{4}\right) \geq 64 \phi_{3}^{2}
$$

Consequently for an acoustic spectrum case the condition - that the band edge plane wave frequency is repelled from the linear spectrum with increasing amplitude - is only necessary but not sufficient for a tangent bifurcation to occur. It is interesting to note that condition (10.22) has been obtained with the help of multiple scale expansions already in 1972 by Tsurui [184] and more recently by Flytzanis, Pnevmatikos and Remoissenet 92 for systems with $v_{\mu}=0$.

\section{Symmetry breaking}

At the bifurcation of the plane wave new periodic orbits occur. Because the bifurcation is tangent, the new orbits have the same period as the plane wave orbit (at the bifurcation point). Any periodic orbit is a closed loop in the phase space of the system. Consequently the new bifurcating orbits can be obtained by deformations of the loop corresponding to the plane wave orbit at the bifurcation. Flach has proven [80] that there is no possibility to simultanously deform the plane wave loop and to keep its invariance with respect to the permutations (10.1). Consequently there are at least $N$ families of periodic orbits bifurcating from the plane wave orbits at a tangent bifurcation. The spatial structure of these orbits corresponds to exactly the spatial structure of a discrete breather [80.

\section{Let us predict!}

What follows from the stability analysis of band edge plane waves? First, if a band edge plane wave undergoes a tangent bifurcation, new periodic orbits bifurcate which i) are not invariant under discrete translations, ii) have a spatial shape of one discrete breather. Second, we know that for systems given by (3.1) and an optical-like spectrum $\left(v_{2} \neq 0\right)$ the repelling of the band edge plane wave frequency with increasing amplitude is a sufficient condition for the tangent bifurcation to occur! For acoustic-like spectra $v_{2}=0$ the repelling condition is necessary but not sufficient.

That enables us to predict the existence of discrete breathers - without actually finding the solutions. A wellstudied example is the FPU lattice in one dimension (acoustic linear spectrum). If we choose $\phi_{4}>0$ and increase $\left|\phi_{3}\right|$ starting from zero, we will loose breathers which bifurcate from the upper band edge plane wave - exactly when (10.22) is violated. That has been demonstrated by Sanduski and Page by performing careful numerical experiments [156]. Another prediction: there exist no discrete breathers in a Toda chain (this is an integrable system with nearest neighbour interaction $\Phi(z)=\left(\mathrm{e}^{-z}+z-1\right)$ and without on-site potentials $V(z)=0$ [181], thus the linear spectrum is acoustic). Indeed, expanding the Toda interaction $\Phi(z)$ we obtain $v_{\mu}=0, \phi_{2}=1, \phi_{3}=-1 / 2, \phi_{4}=1 / 6$. Consequently (10.22) is violated. Indeed numerical efforts to obtain discrete breathers in a Toda lattice have been fruitless [118], 8 . 
If we consider a system with an optical-like spectrum, we see that the repelling of the plane wave frequency from the linear spectrum with increasing amplitude is sufficient. Consider now a narrow linear spectrum. Then in general (note that exceptions apply) either both band edge plane wave frequencies are increasing with increasing amplitude, or they are decreasing. Thus usually one of the band edge plane waves will fullfill the repelling criterion and one will not. The plane wave which fulfills the repelling criterion thus gives rise to discrete breathers! We obtain another way of understanding the existence proof of MacKay and Aubry [137] where the antiintegrability limit was nothing else but the limit of a narrow optical-like linear spectrum! Further we can predict that when the Toda lattice is modified by e.g. alternating heavy and light masses, one changes the linear spectrum and obtains in addition to the acoustic band an optical band. Then discrete breathers should exist which bifurcate from one of the edges of the optical band. Indeed numerical simulations have demonstrated the existence of discrete breathers in the Toda lattice with alternating masses!

\section{LATTICE DIMENSION EFFECTS}

In the first part of this section we will give some general remarks on dimension effects on discrete breathers. In a second part we will discuss recent results by Flach, Kladko and MacKay [83, who show a profound effect of the lattice dimension on energy properties of DBs. Namely energies of DBs in two- and three-dimensional lattices have nonzero lower bounds. In contrast one-dimensional lattices can allow for DBs with arbitrary small energy values.

\section{A. General remarks}

As it follows from the preceeding analysis the existence of discrete breathers is not crucially related to the dimension of the lattice. Thus we are dealing with localized excitations which can exist e.g. in one-, two- and three-dimensional lattices. Consequently the dimension of the lattice can have only an effect on the properties of discrete breathers, but apparently not on their existence.

As we discussed in section IV the dimension can affect the spatial decay of breathers which bifurcate from acoustic band edge plane waves. This happens if the system does not possess a symmetry $H(x)=H(-x)$, because in that case even Fourier number components are excited in the breather solution. The dc component will then resonate with the lower band edge of the acoustic band of the linear spectrum. As we conjectured, the spatial decay of the dc component will be then alebraic instead of exponential. The power of the algebraic decay will depend on the lattice dimension.

\section{B. Energy thresholds for discrete breathers}

The tangent bifurcation analysis of band edge plane waves in section $\mathrm{X}$ has been done for finite one-dimensional lattices. The analysis can be carried out in higher dimensional lattices 80]. For convenience assume that we are discussing a $d$-dimensional hypercubic lattice with periodic boundary conditions and a total number of sites $N$. Then the critical amplitude of the band edge plane wave at the bifurcation is given by

$$
Q_{q} \sim N^{-1 / d},
$$

i.e. the critical amplitude is inverse proportional to the linear size of the system. This happens because the distance between the eigenvalues of the linear spectrum at a band edge are inverse proportional to the squared linear size of the system 80]. Let us assume that the energy of a solution is essentially quadratic in the amplitudes for small amplitudes. It follows for the critical energy of the plane wave at the bifurcation point 80]

$$
E \sim N^{1-2 / d} .
$$

Note that this energy is not to be confused with the one-particle energies $E^{c}$ (or energy densities) from section $\mathrm{X}$. The energy of the bifurcating new periodic orbits have to be the same as (11.2) at the bifurcation. Result (11.2) is surprising, since it predicts that the energy of a discrete breather for small amplitudes should diverge for an infinite lattice for $d=3$ and stay finite (nonzero) for $d=2$, whereas for $d=1$ the discrete breather energy will tend to zero (as initially expected) in the limit of small amplitudes and large system size. Because the energy of a discrete breather can not be zero for nonzero amplitudes of the breather center, it follows that DB energies have nonzero lower bounds for two- and three-dimensional lattices. That finding can be extremely important for any experimental application. 
Let us estimate the discrete breather energy in the limit of small amplitudes and compare the result with (11.2). Denote the largest amplitude of a given DB by $A_{0}$ where we define that the site $l=0$ is the one with the largest amplitude. Then the amplitudes away from the breather center will decay in space according to an exponential law $A_{l} \sim A_{0} \mathrm{e}^{-\delta|l|}$. To estimate the dependence of the spatial decay exponent $\delta$ on the frequency of the time-periodic motion $\Omega_{b}$ (which is close to the edge of the linear spectrum) it is enough to consider the dependence of a frequency of the linear spectrum $\Omega_{q}$ on the wave vector $q$ when being close to the edge. Generically this dependence is quadratic $\Omega_{E}-\Omega_{q} \sim\left|q-q_{E}\right|^{2}$ where $\Omega_{E} \neq 0$ marks the frequency of the edge of the linear spectrum and $q_{E}$ is the corresponding edge wave vector. Then analytical continuation of $\left(q-q_{E}\right)$ to i $\left(q-q_{E}\right)$ yields a quadratic dependence $\left|\Omega_{b}-\Omega_{E}\right| \sim \delta^{2}$. Finally we need to know how the detuning of the breather frequency from the edge of the linear spectrum $\left|\Omega_{b}-\Omega_{E}\right|$ depends on the small breather amplitude. This is done using perturbation theory for weakly nonlinear oscillators 143. For that we need to know the exponent $(\mu-1)$ of the first nonlinear term in the equation of motion for the band edge plane wave for small amplitudes (see (10.7) and (10.8) as an example). Note that we slightly changed the definition of $\mu$ as compared to 83]. Depending on $\mu$ we obtain $\left|\Omega_{b}-\Omega_{E}\right| \sim A_{0}^{z}$ where $z=\mu-2$ for even $\mu$ and $z=2 \mu-4$ for odd $\mu$.

Now we are able to calculate the energy of the discrete breather replacing the sum over the lattice sites by an integral

$$
E_{b} \sim A_{0}^{2} \int r^{d-1} \mathrm{e}^{-\delta r} \mathrm{~d} r \sim A_{0}^{(4-z d) / 2} .
$$

We find that if $d>d_{c}=4 / z$ the breather energy diverges for small amplitudes, if $d=d_{c}$ the DB energy stays finite and nonzero for small breather amplitudes, and for $d<d_{c}$ the DB energy tends to zero for small amplitudes. The tangent bifurcation analysis was performed using $\mu=3,4$. Inserting $\mu=3,4$ we obtain $d_{c}=2$ which is in accord with the exact results on the plane wave stability 80 ].

An immediate consequence is that if $d \geq d_{c}$ the energy of a breather can not be zero, i.e. there is a nonzero lower bound for DB energies. This happens because for any finite amplitude the breather energy can not be zero. We obtain an energy threshold for the creation of discrete breathers for $d \geq d_{c}$. This new energy scale is set by combinations of the expansion coefficients in (3.1).

Another consequence is that for $d \geq 2$ the energy threshold of DBs is always nonzero, independent of the value of $\mu$ (note that by definition $\mu \geq 3$ ). A one-dimensional system can have nonzero lower energy bounds for DBs only if $\mu \geq 5$.

Numerical calculations of discrete breathers have been performed in 83 in order to test these results. Discrete breathers were numerically continued starting from large energy breathers which were strongly localized. The method chosen was the steepest descent method, using phase space variables. The dimension of the lattice was $d=1,2,3$. A typical size for a three-dimensional lattice was $30 \times 30 \times 30$. Details can be found in 83. In Fig. 11 we show the dependence of the DB energy on the DB amplitude for a DNLS system with $\mu=4$. Clearly the energy thresholds are observed. Other Hamiltonians have been also successfully tested. The analysis of the DB solution which corresponds to a minimum of energy shows that these breathers are still strongly localized on a few lattice sites [83].

The existence of a minimum of the energy on the one-parameter family of DB solutions has further consequences. It immediately follows that at the minimum energy solution a saddle-node bifurcation appears, which is responsible for the fact that no DB solutions exist below the threshold energy. Consequently either DB solutions with smaller or larger amplitudes have to be dynamically unstable! Since in the case of a nonzero energy threshold the DB solutions increase in energy as the amplitude is decreased, a similar analysis of the tangent bifurcation of the band edge plane wave leads to the conclusion that the DB branch with smaller amplitudes is dynamically unstable, whereas the one with larger amplitudes (with respect to the amplitude which extremizes the DB energy) will be dynamically stable. These conclusions support also the fact that the steepest descent method is insensitive against instabilities of DB solutions.

\section{DISCRETE SYMMETRIES}

In this section we will discuss the relation between discrete breathers and discrete symmetries of the equations of motion. We will not discuss continuous symmetries because the only continuous symmetry in the case of (2.1) is the symmetry with respect to shifts in time, i.e. any solution $X_{l}(t)$ will generate another solution $X_{l}(t+a)$. For convenience and practical purpose we consider Hamiltonian problems, which usually imply that the trajectories in phase space defined by $X_{l}(t)$ and $X_{l}(t+a)$ are identical, i.e. $t \rightarrow(t+a)$ corresponds to a shift along the trajectory. Note that this is not the case for e.g. damped equations of motion (then the equations still have the continuous symmetry, but the trajectories in phase space are not invariant). 
Then the only symmetries we are left with are discrete symmetry operations. E.g. (3.1) is invariant under time reversal $t \rightarrow-t$. Another important discrete symmetry is the discrete translational symmetry. In addition we can have optional symmetries due to the lattice properties and a parity symmetry (displacement reversal) $u_{n} \rightarrow-u_{n}$ for all $n$ (the last one happens in (3.1) if $\phi_{\mu}=v_{\mu}=0$ for all odd $\mu$ ). All these symmetries imply that when the corresponding symmetry operation is applied to a given solution, a new solution of the equations of motion is obtained. Each symmetry can be represented as a function acting on the phase space of the system. These functions map the phase space into itself. The relevant question is then, whether a given trajectory in phase space is invariant if the symmetry function is applied, or not. For a trajectory to be invariant means that all points of the trajectory when transformed according to the symmetry function are again points on the same trajectory.

Note that since we are solving nonlinear equations in the phase space variables, the invariance of a trajectory under a symmetry operation is not granted at all. This is different from linear equations which yield eigenvalue problems. Then the corresponding eigenvectors will always reflect the symmetry of the equations (of the matrix). Exceptions can occur when there are degeneracies (e.g. traveling wave solutions $\cos (i(k n-\omega t))$ of a linear lattice with periodic boundary conditions are not invariant under time reversal: this is due to degeneracies with respect to $\pm k$, which allows for symmetric and antisymmetric standing waves (invariant under time reversal) and travelling waves with $\pm k$ (not invariant under time reversal).

Discrete breathers correspond to trajectories in phase space which are not invariant under discrete translations along the lattice. Consequently we can generate new discrete breather solutions by applying discrete translations. The Fourier coefficients $A_{k l}$ describing the solution are required to fullfill

$$
A_{k l}=A_{-k, l}^{*},
$$

were $A^{*}$ denotes the complex conjugate of $A$. In general the solutions do not have to be invariant under time reversal. MacKay and Aubry apply the existence proof of DBs however to DB solutions which are invariant under time reversal (in the case when the equations are invariant). Also all practical calculations performed so far have actually been dealing with DB solutions invariant under time reversal. This implies in addition to (12.1), that for solutions invariant under time reversal there exists an origin of time such that

$$
A_{k l}=A_{-k, l},
$$

i.e. that all Fourier coefficients are real.

Another restriction on the Fourier coefficients is obtained, if the equations are invariant under displacement reversal, and if we look for solutions which are also invariant under displacement reversal. Then it follows that

$$
A_{k l}=0, \quad k=0,2,4,6, \ldots .
$$

This can help to reduce computation time for finding numerical DB solutions. The same applies to lattice reflection symmetries. However one should bear in mind that in principle DB solutions can lack these discrete symmetries, just as they lack the discrete translational symmetry!

Let us mention some related results. First MacKay and Aubry [137] explain that DB solutions in the antiintegrable limit can be obtained for i) systems without discrete translational symmetry (disorder); ii) Hamiltonian systems without time reversibility. Further the existence of phase-locked DB solutions systems with damping and timeperiodic driving will be briefly discussed in section XIX. These DB solutions are obtained for systems which are not invariant under time reversal and even under continuous time translations (the continuous time translation is replaced by a discrete one)!

If one is looking for moving breather solutions, then the solutions can not be invariant under time reversal. It could thus be that exact moving breather solutions can occur through bifurcations from plane waves which are not band edge plane waves (this is so because typically band edge plane waves are not degenerate in the linearized equations, and thus band edge plane waves keep the time reversibility of the system).

As a result we can conclude that discrete breather solutions are not only quite robust under system perturbations which leave the systems symmetries unchanged, but discrete breathers apparently are quite common among systems with different symmetry properties.

\section{A CONCEPTUAL APPROACH}

Very often one is dealing with a rather complicated system (as a crystal with several atoms per unit cell), so that it appears to be rather complicated to check numerically whether discrete breathers exist. It amounts to taking into account e.g. about ten degrees of freedom per unit cell (this number can easily increase to twenty!). Solving then 
for spatially localized oscillations can become a very hard numerical task. It is then important to have a conceptual approach of analyzing the system without actually solving for DBs. As a result we want to be able to predict the existence of discrete breathers.

This can be accomplished using the fact that DB solutions bifurcate from band edge plane waves. In the following we will explain this approach.

First one has to solve the linearized equations of motion - still a complicated task. This has been done using either numerical diagonalizations, but one can also use results of scattering experiments (usually neutron scattering, but that is up to the experimental setup). We then know the linear spectrum of the system.

The linear spectrum has to be analyzed in order to find all allowed frequency regions for potential discrete breather solutions by taking into account the nonresonance condition of all harmonics of a DB with respect to the linear spectrum.

After accomplishing that task, we should try to find narrow optical bands in the linear spectrum, whose surrounding forbidden frequency regions belong to the allowed frequency range of a discrete breather. Call these bands selected optical bands. Here narrow implies that the width of those bands is smaller than their absolute position on the frequency axis.

Now we have to make some estimate on the nonlinearities in the system. Especially we need nonlinearities in the normal mode variables of the linearized system which correspond to the band edges of the selected optical bands. We need only a perturbative treatment of the nonlinear part which is valid for small amplitudes.

Next we have to calculate the change of the frequency of the band edge normal modes of the selected optical bands in perturbation theory with increasing amplitude. We have to select the band edges whose plane wave frequencies are repelled from the selected optical band with increasing amplitude.

Now we can expect that discrete breathers exist with frequencies in the gaps of the linear spectrum which touch the selected band edges of the selected optical bands. The spatial symmetries of these breathers should correspond to the eigenvectors of the normal modes of the selected band edges.

We should take into account that for two-dimensional systems (e.g. surfaces) or three-dimensional systems these discrete breathers will have nonzero lower energy thresholds.

\section{PLANE WAVE SCATTERING BY DISCRETE BREATHERS}

In this section we will discuss results on the scattering of plane waves by discrete breathers. There are only few results known, and certainly there remains much to be done in this field. First we will discuss small amplitude lattice plane wave scattering. This problem is closely tied to the dynamical stability analysis of DBs. Some numerical results can be found in [89]. Recent results have been provided by Cretegny [48]. In a second approach we will discuss the scattering of electrons by discrete breathers (here the discrete breather is a localized vibration of a crystal lattice which is interacting with electrons). Details can be found in [82].

Conceptually these two cases can be separated with respect to their plane wave frequencies. In the first case (phonons scattered by discrete breathers) the time scale defined by the plane wave excitations is of the same order as the time scale of the DB dynamics. In the second case (electron scattering) the time scale of the electronic plane wave is orders of magnitude shorter than the time scale of the breather dynamics. These differences are crucial for the concepts used.

\section{A. Phonon scattering}

We use the term phonon here only to stress that the plane waves considered here are small perturbations of the discrete breather solution itself. These plane waves are connected to the eigenmodes of the linear stability analysis of a DB.

Not much is known about phonon scattering, neither numerically nor analytically. Up to now the only published results on this topic are for one-dimensional lattices of the type (3.1), which occured in Ref. [89] where a numerical analysis of the transmission of small-amplitude plane waves was performed. The full equations of motion were used in this analysis. The discrete breather solution was obtained with precision $10^{-4}$ in the amplitudes, which limited the observation to transmitted wave amplitudes of the same amplitude. On the other hand the incident plane wave had to have a small enough amplitude in order to avoid nonlinear effects in its propagation besides the interaction with the discrete breather. In particular the superposition should still hold approximately. Thus the incident wave amplitudes were $10^{-2}$. That defined a lower observation threshold on the transmission coefficient $T \geq 0.01$. 
The study was performed for different wave numbers of the incident wave. Reflection and transmission were measured. Reflection was observed due to an increase in the energy density on the incident side of the DB, whereas the transmission coefficient was smaller than 1 . The scanned wavenumber interval was $0.1 \pi<q<0.9 \pi$. The results showed that the transmitted wave intensity was modulated in time (see Fig.12). However this modulation could not be easily related to the internal dynamics of the breather. The amplitude of these modulations was of the order of the averaged transmission itself. The time period of these modulations is of the order of 20 breather periods, more precisely about 280 time units of (3.1)!

The most important result of this study was the exponential decay of the squared transmission coefficient with increasing wavenumber as shown in Fig.13 (triangles). Note that the filled circles represent the time-averaged transmitted intensity, and the exponential decay in $q$ is observed only for the $q$-region studied. Thus discrete breathers appear to strongly reflect phonon plane waves, which can be of importance whenever we consider heat flow. The reflectivity properties are strongly wavenumber dependent.

Further numerical and analytical results have to be obtained. There are several important aspects to be clarified the influence of lattice dimensions, discrete breather properties and of the plane wave wave vector on the scattering. In the following we will present some recent results by Aubry and Cretegny which appear to be very stimulating for further studies.

The idea is to derive the scattering result from the linear stability analysis of a discrete breather. Indeed if we solve the Floquet problem of the linear stability analysis of a discrete breather, we know the eigenvectors of the spatially extended states. We could then combine these eigenvectors in order to obtain a scattering setup - an incident and reflected waves on one side of the obstacle (here the DB) and a pure transmitted wave on the other side. However in order to do so it requires that the eigenvalues of the two eigenvectors are exactly equal. This poses a problem for finite systems, as the discussion in the following shows.

Recall that the linear stability analysis is defined by equation (8.1), which is a set of coupled linear differential equations for the small perturbations $\delta_{l}$, where the coefficients of the equations are functions of the breather solution, and thus time-periodic. The corresponding Floquet map (8.4) defines the eigenvalue problem. Since the Floquet map operates on the phase space, the space dimension is $2 N$ where $\mathrm{N}$ is the total number of degrees of freedom. Because of the symplectic structure of the map there are at most $N$ independent Floquet multipliers. Consider now the case that no breather is excited. Still we can solve the Floquet eigenvalue problem. Assume periodic boundary conditions (PBC). Then the Floquet multipliers are double degenerate. This happens because of the familiar degeneracy of eigenvalues for running waves with opposite wave numbers. The degeneracies are a consequence of the nonabelian group formed by the discrete translational symmetry (a result of periodic boundary conditions) and the reflection symmetry around any lattice site 134]. Note that we can avoid these degeneracies by imposing fixed or free boundary conditions. The PBC are attractive because we want to study travelling waves. However for any finite system the scattering problem is not defined regardless of the boundary conditions.

If we have a breather solution, equation (8.1) is not translationally invariant anymore, even for PBC. As a consequence we are left with an abelian group of reflection symmetry around the center of the DB, which causes all degeneracies in the Floquet spectrum to be removed [134]. All Floquet eigenstates are now strictly symmetric or antisymmetric with respect to the reflection operation. There exists no pair of symmetric and anti-symmetric eigenstates with the same eigenvalues. This holds for any finite system regardless of the boundary conditions.

Far away from the breather location any extended Floquet eigenstate will have the structure of the extended eigenstates of the Floquet map without a DB. Thus for PBC the true eigenstates far away from the breather can be represented schematically as $\left(a\left|q_{+}>+b\right| q_{-}>\right)$, where $\mid q_{ \pm}>$denotes a running wave with wave vector $\pm q$. Note that these running wave vectors are not eigenvectors of the Floquet map without a DB solution, because the eigenvalues will not match. The wave number $q$ is determined from the dispersion relation for the infinite system without a DB solution. The corresponding frequency $\omega(q)$ is determined from the numerically obtained Floquet phase $\theta$ :

$$
\theta=\omega T_{b}+2 \pi m,
$$

where $T_{b}$ is the DB period and $m$ is any integer. The resulting frequencies

$$
\omega=\frac{\theta}{T_{b}}-m \frac{2 \pi}{T_{b}}
$$

have to belong to the linear spectrum $\omega(q)$. In general this allows for several values $\omega_{q}$. However we know that the DB frequency and all its multiples have to be outside the linear spectrum. This limits the number of allowed $q$-values.

If we can find another eigenvector of the Floquet map with the same eigenvalue, we can combine both in an appropriate way to obtain the scattering setup as described above. That is impossible for any finite system because there are no degeneracies left once the DB solution is assumed to exist. What happens in the limit a large system size $N \rightarrow \infty$ ? Suppose the DB solution is itself symmetric ( or anti-symmetric). Then the anti-symmetric (symmetric) Floquet 
eigenstates will be unchanged as compared to the Floquet map without a breather! Consequently the eigenvalues of the anti-symmetric (symmetric) eigenstates did not change either. Thus we have to study the $N$-dependence of the symmetric (anti-symmetric) eigenstates in the presence of the breather. The changes of these eigenstates due to the breather presence give the values for the eigenvalue splittings due to the lifting of the degeneracies.

We can obtain an upper estimate for the splittings. The splittings can not become larger than the averaged spacing, since the spectrum of each subgroup (symmetric and anti-symmetric) is confined by the band edges of the linear spectrum and crossings within each subgroup are not allowed in general. The averaged spacing is of the order $1 / N$ in the middle of the linear spectrum and decreases to $1 / N^{2}$ and the band edges. Consequently the splittings decrease with increasing system size. In the limit $N \rightarrow \infty$ we can find degeneracies and thus construct a scattering case. If (14.2) is fulfilled for more than one frequency $\omega(q)$ then scattering involves several wave numbers.

Cretegny has calculated the transmission coefficient of the case in Fig.13 (solid line). Qualitative and partially quantitative agreement with the numerical simulation of the full system is found. Moreover a decrease of the transmission coefficient at small wave numbers is predicted (this is in analogy to the scattering by a single defect in a linear system, for details see Economou [63]). However this linear approach is not capable of explaining the observed modulations (see above). These modulations appear for (14.2) having exactly one solution $\omega_{q}$. Also finite-size effects can be ruled out. Splittings in the Floquet spectrum will cause some modulations, but the amplitudes should be small, and the estimated modulation period is three orders of magnitude larger than the observed one! Apparently there is still a lot to understand in the scattering problem. Also the solid line in Fig.13 indicates that total reflection is possible for wave numbers $q \approx 2.1$. However the numerical experiment (filled circles in Fig.13) do not indicate any total reflection.

\section{B. Electron scattering}

If a discrete breather is excited in a crystal lattice, we obtain a localized crystal lattice vibration. Often lattice vibrations couple to electronic degrees of freedom. From a mathematical point of view the adding of electronic degrees of freedom corresponds to additional degrees of freedom with eigenfrequencies orders of magnitude larger than the eigenfrequencies of lattice vibrations. This is different from the interaction of a breather with a lattice plane wave (see preceeding subsection).

Let us consider a classical nonlinear lattice which allows for breather solutions. Generally the excitation of the relevant breather degrees of freedom leads to a localized polarization of the lattice. In the classical ground state of this system the polarization vanishes. If we excite a discrete breather, then it will induce a (time-periodic) multipole field at distances large compared to the breather size. Generally the first nonvanishing moment will be a dipole moment. The induced polarization will be spatially localized, in accord with the strong localization properties of the breather solution.

Let us consider the interaction of a single electron with a discrete breather in the case when the distance between the electron and the breather is much larger than the breather size. Since we are describing the lattice degrees of freedom classically, we can use the adiabatic approximation [53]. This means, that the motion of the electron is described by using the positions of the lattice degrees of freedom as parameters. Thus the electron feels a multipole field which originates from the breather. This kind of treatment of the electron is similar to the consideration of electrons in a lattice with defects [114]. The difference is, that i) the breather (dynamical defect) does not posess an uncompensated charge and ii) the breather is slowly (as compared to the inverse electron frequency) changing its multipole field.

Since the multipole field of the breather contains in general a dipole component, we can study the scattering of an electron in a dipole field. We consider the case when the electron can follow a path which does not come close to the breather location. If this assumption is not true anymore, the electron can be trapped by the breather, as will be shown in the next section.

The potential of a dipole in a three-dimensional lattice is given by

$$
V_{d}(\vec{r})=\frac{1}{\epsilon} \frac{\vec{d} \vec{r}}{r^{3}}
$$

Here $\vec{d}$ is the dipole moment induced by the breather (which is actually slowly periodically oscillating with time). The dielectric permeability $\epsilon$ describes the reduction of the dipole field due to polarization effects.

The motion of an electron with isotropic effective mass $m^{*}$ and charge $e$ will be then described by the Hamiltonian $H$ and the wave function $\Psi(\vec{r}, t)[114$

$$
H=-\frac{\hbar^{2}}{2 m^{*}} \Delta+e V_{d}(\vec{r}), i \hbar \frac{\partial \Psi}{\partial t}=H \Psi .
$$


The dipole potential (14.3) does not posess localized states. This can be easily determined by considering the corresponding classical motion in the potential (14.3). Clearly there exist no periodic orbits having some finite distance from the potential center $\vec{r}=0$. Thus there appear no localized electron states which are weakly localized as compared to the breather size. This situation is opposite to the Coulomb field problem where periodic orbits exist and lead to the existence of hydrogen like localized states, as used in the description of Wannier excitons. To find trapped electronic states induced by a discrete breather we have to take into account the internal breather structure, which will be studied in the next section.

To account for the elastic electron reflection in the dipole potential (14.3]) we can use Born's approximation [53], which holds if the interaction energy between the electron and the breather will be small compared to the kinetic energy of the electron. Denoting by $\mid \vec{k}>$ the plane wave states of the electron in the absence of a breather, we have to calculate the matrix elements

$$
<\vec{k}\left|V_{d}\right| \vec{k}^{\prime}>=\int \mathrm{e}^{i\left(\vec{k}-\vec{k}^{\prime}\right) \vec{r}} V_{d}(\vec{r}) \mathrm{d} r^{3}
$$

Straight forward integration gives

$$
<\vec{k}\left|V_{d}\right| \vec{k}^{\prime}>=-i \frac{4 e \pi}{\epsilon} \frac{\vec{d}\left(\vec{k}-\vec{k}^{\prime}\right)}{\left|\vec{k}-\vec{k}^{\prime}\right|^{2}} \delta\left(E_{\vec{k}}-E_{\vec{k}^{\prime}}\right) .
$$

The electronic energies $E_{\vec{k}}$ measure the energy of the incoming and outgoing plane waves. All other quantities related to the electron scattering in the used approximation can be obtained from these matrix elements.

In the nongeneric symmetric case that the breather does not posess a dipole moment, the quadrupole field tensor $D_{\alpha \beta}$ has to be considered (note that we use the definition $D_{\alpha \beta}=\sum_{i} e_{i} x_{\alpha}^{(i)} x_{\beta}^{(i)}$ ). The corresponding potential is given by

$$
V_{q}(\vec{r})=\frac{1}{2 \epsilon} D_{\alpha \beta} \frac{\partial}{\partial x_{\alpha}} \frac{\partial}{\partial x_{\beta}} \frac{1}{r}
$$

Again there are no bound states in potential (14.7) as in the dipole case. The matrix elements can be obtained by integrating:

$$
<\vec{k}\left|V_{q}\right| \vec{k}^{\prime}>=-i \frac{2 e \pi}{\epsilon} \frac{D_{\alpha \beta} k_{\alpha} k_{\beta}}{\left|\vec{k}-\vec{k}^{\prime}\right|^{2}} \delta\left(E_{\vec{k}}-E_{\vec{k}^{\prime}}\right)
$$

\section{TRAPPING OF PLANE WAVES BY DISCRETE BREATHERS}

In the preceeding section we discussed the scattering of plane waves by discrete breathers. A natural next question would be, whether these plane waves can be trapped by the discrete breather. In other words, we look for bound states of the medium which provided the plane waves to be scattered by a discrete breather. This is not to be confused with the discrete breather solution itself - which is a bound state too, but not due to an obstacle which is breaking the translational symmetry.

We will first discuss trapping of phonons. These bound states will be closely connected to the discrete breather solution itself, as well as to its dynamical stability properties. Then we will discuss the trapping of electrons by breathers. We will review results of Flach and Kladko [82], Aubry [13] and Vekhter and Ratner [186], [187], and add some thoughts.

\section{A. Phonon trapping}

We consider a discrete breather solution. Then we add small perturbations to the initial conditions and ask whether we can find bound states for the perturbations. The task is thus again to analyze the linearized stability problem (8.1). First we know that the DB solutions come in one-parameter families. Thus equation (8.1) will always have two eigenvectors with Floquet multipliers both equal to +1 . This is so because the one-parameter family of DB solutions (which are time-periodic and thus one-dimensional manifolds in phase space) is of dimension two. Given any point $P$ on this manifold, we can always find two orthogonal directions (no matter what scalar product we use) which are 
tangent to the manifold in $P$. For instance one direction is simply pointing in the direction of the periodic orbit which is defined by $P$. The second one will point to neighboring periodic orbits.

Both eigenvectors will be exponentially localized on the lattice, because they correspond to differences of two neighbouring DB solutions on the manifold. Thus formally we can call them bound phonon states. This is another interpretation of the same fact that discrete breathers come in one-parameter families. The term 'bound state' implies here that we can add energy to the DB solution such that the additional energy is not dispersed over the entire lattice.

Another possibility is that the eigenvalue problem (8.1) allows for additional spatially localized eigenstates. If the Floquet multipliers of these eigenstates are located on the unit circle, i.e. if these perturbations are linearly stable, then we obtain bound states which are not merely a consequence of the existence of the DB solution itself. Such eigenstates were observed in many numerical studies [157], [46], [52], [87], [90], [88], 84], and especially studied systematically by Marin and Aubry in [140].

What happens if we consider the full nonlinear equations instead of the linearized phase space flow around a breather solution? The two-dimensional manifold of one-parameter DB families is still present, so that the first type of bound states survives - but of course these bound states are somehow trivial. The second nontrivial type of bound states would if it survives correspond to quasiperiodic breathers. Generally we do not expect them to exist (see section IV). Strictly speaking we are then left with the first type of trivial bound states. However as also discussed in sections III and VIII, the life-time of the second type of bound states can be very large compared to the time periods of the DB solution.

\section{B. Electron trapping}

As already indicated in the previous section, the treatment of electrons interacting with discrete breathers is quite complicated. We will have to make a lot of additional assumptions in order to come to published results. First we have to consider just one electron moving on a lattice - so we do not know the effect of electron-electron interaction [95] on what is to come. Next we are not aware of any rigorous treatment of both lattice and electronic degrees in a quantum mechanical way. So what we will consider is one electron whose motion is described within a tight binding model. The electron will be coupled to a lattice of classical interacting degrees of freedom.

\section{Model structure}

The electron description in a tight-binding representation implies that at every lattice site we can find a complex probability amplitude $\Psi_{l}(t)$ such that $\left|\Psi_{l}(t)\right|^{2}$ gives the probability distribution for the electron. The tight-binding Hamiltonian for the electron is then

$$
H_{e}=\sum_{l}\left[\epsilon_{l} \Psi_{l} \Psi_{l}^{*}+T_{l}\left(\Psi_{l} \Psi_{l+1}^{*}+c c\right)\right] .
$$

Here $T_{l}$ is some hopping matrix element, and $\epsilon_{l}$ is a one-site energy. The equations of motion are given by

$$
\gamma \dot{\Psi}_{l}=i \frac{\partial H_{e}}{\partial \Psi_{l}^{*}}
$$

Here the parameter $\gamma$ regulates the frequency scale of the electronic system. Thus the consideration of just one electron reduces the whole quantum mechanical task for the electron to a classical Hamiltonian problem (15.1),(15.2) where we have besides the energy another integral of motion - the number of electrons $N_{e}$

$$
N_{e}=\sum_{l}\left|\Psi_{l}\right|^{2} .
$$

The coupling to the classical variables $u_{l}(t)$ of (3.1) is then obtained by assuming a certain functional form of the parameters $\epsilon_{l}\left(\left\{u_{m}\right\}\right)$ and $T_{l}\left(\left\{u_{m}\right\}\right)$. Usually the coupling has some locality properties. Consequently we arrive at some extended lattice problem - instead of only (3.1) we have in addition (15.1), which increases the number of degrees of freedom per lattice site. As we already pointed out, the number of degrees of freedom is not crucial for the existence of discrete breathers. Thus we can search for discrete breathers in the extended system with an electron, and if we find solutions, they will correspond to both a vibrational localization of the lattice as well as a localization of the electron. 


\section{Holstein model and nonlinear generalizations}

The original Holstein model is obtained by setting $v_{2}=1, v_{\mu \neq 2}=\phi_{\mu}=0$ in (3.2), (3.3), and $T_{l}=T, \epsilon_{l}=u_{l}$. Aubry considered a nonlinear generalization of this model by allowing $V(z)$ of (3.2) to be any anharmonic potential (Aubry still requires $\Phi(z)=0)[13]$.

The Holstein model has been used to discuss polaron formation. The polaron is a groundstate solution of the electron-lattice system which is not invariant under discrete lattice translations. Moreover the polaron solution corresponds to a localized electron combined with a localized static displacement field. The polaron is indeed often a consequence of an interaction $\epsilon_{l}=u_{l}$ because this interaction term shifts the equilibrium position of the $u_{l}$ variables in the presence of some nonzero electronic density. Let us consider the polaron solution for the original Holstein model. There $V(z)=u_{l}^{2} / 2$. The condition $\dot{u}_{l}=0$ leads to

$$
u_{l}=-\left|\Psi_{l}\right|^{2}
$$

Then we obtain a DNLS for the electronic amplitudes

$$
\gamma \dot{\Psi}_{l}=i\left[-\left|\Psi_{l}\right|^{2} \Psi_{l}+T\left(\Psi_{l-1}+\Psi_{l+1}\right)\right] .
$$

We have already discussed this equation. It allows for breather solutions. Consequently the polaron groundstate is exponentially localized. Once the groundstate is not invariant under translations, so will be other trajectories which can be regarded as excitations above the given groundstate. Because of the localization properties of the groundstate some excitations above the groundstate may stay localized too. This is in complete analogy to the excitations above a kink groundstate in a kink-bearing one-dimensional lattice 29. All we have to check is whether the spectrum of the localized excitations is not in resonance with the linear spectrum of the extended excitations above the polaron groundstate. This is in complete analogy to the discrete breather analysis.

Aubry indeed proves the existence of localized excitations above the polaron groundstate by analytical continuation from the antiintegrability limit $T=0$ of the nonlinear generalization of the Holstein model $[13]$. For $T=0$ the lattice sites are not interacting, and the solution at one lattice site with $\left|\Psi_{l}\right|^{2}=\rho$ reads

$$
\begin{array}{r}
u_{l}(t)=G\left(t ; \omega_{b}, \alpha\right)=G\left(\left(t+2 \pi / \omega_{b}\right) ; \omega_{b}, \alpha\right), \ddot{G}=-V^{\prime}(G)-\rho \\
\Psi_{l}(t)=\rho^{1 / 2} \mathrm{e}^{i\left(\beta+\gamma^{-1} f_{l}(t)\right)}, f_{l}(t)=\int_{0}^{t} u_{l}\left(t^{\prime}\right) \mathrm{d} t^{\prime}
\end{array}
$$

Here $\omega_{b}$ is the frequency of the $l$-th oscillator and $\alpha$ is a phase. Since for $\rho \neq 0$ the $u_{l}(t)$-dependence will show up with a nonvanishing dc term $\overline{u_{l}(t)}$, the solution $\Psi_{l}(t)$ is given by

$$
\Psi_{l}=\xi_{l}(t) \mathrm{e}^{\left.i \overline{u_{l}(t)} / \gamma\right) t}, \quad \xi_{l}(t)=\xi_{l}\left(t+2 \pi / \omega_{b}\right)
$$

Consequently the lattice variable $u_{l}$ is strictly periodic and its Fourier series representation contains all multiples of the fundamental frequency $\omega_{b}$. The electronic probability amplitude is quasiperiodic, but its Fourier series representation is still an equidistant spectrum with frequencies $\left(\overline{u_{l}(t)} / \gamma+k \omega_{b}\right)$. This should make it easy to escape resonances with the linear spectrum of our problem. The linear spectrum for $T \neq 0$ is given by a degenerate value $\sqrt{v_{2}}-$ the lattice contribution, and a band of width $T / \gamma$ located at the origin - the electronic tight binding band. For small values of $T$ (independent of the value of $\gamma$ ) we can indeed escape resonances of both solution parts with the linear spectrum (note that we have to use polarization vectors here to describe the linear spectrum - consequently the dc part of the lattice contribution does resonate with the electronic band, but due to the orthogonality of the polarization vectors these resonances do not prevent the argument for localization from being valid).

The exponent of the spatial localization of the electron depends essentially on the distance of the electron's frequency from the electronic band. This distance can be chosen to be large for large $u_{l}$ amplitudes, which is then in principle even possible for finite values of $T$. Thus dynamical excitations above a polaron groundstate are possible where the whole excitation energy is concentrated on a few lattice sites.

\section{Related models}

Flach and Kladko have studied a model different from the Holstein model [82]. There the one-site energies are dropped completely $\epsilon_{l}=0$ and the coupling between the electron and the lattice is given by $T_{l}=1+\beta_{1}\left(u_{l}-u_{l+1}\right)$. Also the interaction potential on the lattice $\Phi(z) \neq 0$. Adiabatic approximation (which is here essentially a separation 
of time scales) was used. The lattice itself was assumed to posess discrete breather solutions in the absence of any coupling $\beta_{1}=0$. Then there exist bound electron-breather states for $\beta_{1} \neq 0$ where the electron can be strongly localized even for small values of $\beta_{1}$. Again that is accomplished in the limit of large amplitude DB solutions, which are also strongly localized.

Vekhter and Ratner [186], 187] have studied numerically a similar model. There a bound electron-breather state was found. These numerical studies showed several interesting features (e.g. a weak radiation of the electron out of the localized object) which deserve further investigation.

\section{Bound states without polarons}

All above discussed models tend to the formation of polarons (we know that for the Holstein model, and suspect it for the other models). This is because the interaction term is proportional to $u_{l}$. What happens if we choose e.g. a Holstein model with $\epsilon_{l}=u_{l}^{2}$ ? There will be no polaron solution, so the groundstate of the system with $T \neq 0$ is invariant under translations. However we can easily use Aubry's antiintegrability scheme [13] and find localized solutions in the coupled system for $T$ smaller than some finite values. Indeed for $T=0$ there is only one change in (15.7) namely $f_{l}(t)=\int_{o}^{t} u_{l}^{2}\left(t^{\prime}\right) d t^{\prime}$. Again we find that both parts $u_{l}(t)$ and $\Psi_{l}(t)$ have Fourier time series corresponding to an equidistant spectrum. Again we can easily avoid resonances with the linear spectrum. These solutions will be very similar to the discrete breathers discussed before, in fact these solutions are discrete breathers. Thus the trapping of an electron by a discrete breather is also a slightly irritating term, which is helpful in understanding the physics, but irritating in making mathematical connections. If the presence of an electron creates a polaronic groundstate, then it should be of no surprise that there exist localized excitations above the groundstate (one can still have a hard time in showing that). If the presence of the electron does not create a polaronic groundstate, localized excitations can still exist, because the coupling between the electron and the lattice is already a nonlinear term in the equations of motion.

\section{DISORDER AND NONLINEARITY}

So far we discussed the existence of discrete breathers in systems which are invariant under discrete translations. In this section we will briefly discuss the effect of disorder on discrete breathers. The reader will find additional information in a recent review of Sievers and Page $[162$.

Let us first consider the effects one isolated impurity will have. Using the antiintegrability scheme it follows that discrete breathers of networks of coupled oscillators allow for breather solutions also in the presence of a defect [137]. In other words an isolated impurity will have no serious impact on the existence of DB solutions. Of course the DB solutions will smoothly change, where the changes will occur locally at the defect site. There will be also smooth changes in all properties of DB solutions as linear stability, scattering, bound states etc. These statements are of course true only if the system without a defect is nonlinear.

It is well-known that a linear system with a defect allows for localized solutions too. Because of the linearity the defect problem is reduced to the diagonalization of a matrix. If the defect strength is large enough some eigenvectors (at least one) will be exponentially localized on the lattice. What happens to these localized solutions if nonlinear terms are present? It was long believed that in general nonlinearities destroy the local defect modes. That belief stems from the observation that any nonlinearity is equivalent to an interaction between the normal modes of the linear system. Consequently it was believed that this interaction will cause the energy of the local defect mode to be dispersed among all other extended normal modes. This heuristic argument is indeed true for most trajectories in the phase space of the system. That must be so because typically nonlinearities destroy integrability and cause the phase space to be densely filled with an Arnold web of chaotic trajectories [154], [43]. The trajectories in this web will come close to any point in phase space in the course of evolution. That circumstance is nothing but the celebrated ergodic property of a nonintegrable system. Trajectories from this web can not be localized in some subpart of the phase space then. However the same argument can be applied to the cases we discussed before - most of the systems allowing for DB solutions are nonintegrable. The reason why DB solutions can exist (apart from the nonresonance condition they have to fullfill) is that typically any nonintegable Hamiltonian system will have a phase space also densly filled with periodic trajectories. These trajectories are not ergodic of course. Thus the above given heuristic expectation of the loss of localized defect modes due to nonlinearities has to be corrected. If a localized mode exists in the linear system its frequency will be located outside the linear spectrum of the extended eigenmodes. The frequency of the mode does not change as we change the amplitude of the mode - as a result of the linearity of the system. However the energy of an eigenmode surely changes with amplitude. Bambusi [16] has shown that the continuation of periodic 
orbits can be done by considering orbits with fixed energy (as opposed to fixed frequency). Adding nonlinearities will then cause the periodic orbit to be slightly deformed, but still to exist! In other words, a local defect mode will surely survive the presence of nonlinearities. We can be sure about that because the continuation of the linear defect mode into the nonlinear regime does not meet the problem of resonances. Of course we have to test first whether all higher multiples of the local mode frequency do not resonate with the linear spectrum of extended states. It would be of interest to test whether the continuation of such a mode into the nonlinear regime and a subsequent removing of the defect strength continuously transforms the local defect mode into a discrete breather. We suspect that cases exist where it indeed will happen.

There exist studies of the interaction of a moving breather-like entity with defects. Since we do not know precisely how to treat the moving object, it is again hard to judge the results of the numerical studies [188], [128], [52], [171], [172], 109, 180], 105], 93]. Observations range from trapping of moving DBs by a defect to reflection of DBs by a defect

An even more complicated problem arises if the system is complelety disordered, e.g. that there is a finite density of defects (see also [100]). The linear problem is nothing but the Anderson localization [2]. Although this problem has been widely studied, the methods to prove nontrivial statements are quite elaborate [2], essentially because it is hard to treat the disorder. Adding nonlinearities makes the problem even more complicated. Albanese et al [3], [5], [4] and Frohlich et al 94 studied the nonlinear problem with disorder. Albanese et al prove the existence of periodic orbits in the disordered DNLS where the orbits are exponentially localized on the lattice. MacKay and Aubry [137] can use their continuation scheme for any system of coupled oscillators (with disorder incorporated). The continuation of periodic orbits which correspond to one-site breathers in the ordered lattice is straightforward. But to obtain the exponential localization on the lattice, a notion of distance in the space of periodic orbits is needed and a restriction of the coupling to be of exponential decay itself on the lattice (see also [104]).

\section{QUANTUM BREATHERS}

So far we were discussing properties of coupled nonlinear differential equations. In terms of physics we were discussing classical many-particle systems (lattices). Often the classical description of a physical problem can be an approximation to the quantum mechanical (QM) properties of the system. Then it is legitimate to ask what is the trace of the classical discrete breather in the quantum mechanical description. For continuum problems these questions have been studied e.g. in [49], 98].

First we have to specify the correct correspondence relation between a classical model and its quantum mechanical counterpart. This will be the conventional first or second quantization procedures. There exists some arbitrariness, because the classical variables commute. Consequently there exist families of QM models which yield the same classical model in the classical limit. In the cases studied this degeneracy was not important.

What is a quantum breather? We are interested in eigenstates to the Hamiltonian. Since the QM systems we are looking for are translationally invariant, and the QM eigenvalue problem is by definition a linear problem, we can not expect that spatially localized eigenstates exist. On the contrary all eigenstates will be delocalized in space. How can that be connected to the existence of classical DB solutions? A possible way is that quantized DB solutions can tunnel from site to site. Then in the classical limit the tunneling rate will vanish, and we obtain classical DB solutions. That also implies that in the classical limit we expect to find certain groups of eigenstates (with exactly $N$ members where $N$ is the number of lattice sites) where the eigenvalues in each group are degenerate. Yet another way of saying the same is that in the classical limit we expect to find zero-width bands of $N$ eigenstates. Away from the classical limit we expect these bands to broaden, but not to change in some qualitative way to be specified later.

Because of the bosonic commutation relations we can thus define quantum breathers as bound boson states. A quantum breather state belongs to a band of $N$ states. Each bound state of such a quantum breather band is characterized by a quantum number - a wave number. The particle property of such a bound state can be probed with the help of correlation functions. These correlation functions should show (exponential) decay in the distance between parts of splitted bounded bosons.

There exist several studies where classical DB solutions are quantized using various approximations [161, [168], 124] to obtain estimations on the gap and width of breather bands.

The existence of quantum breathers raises two questions. The first one is: what QM models allow for quantum breathers, and is there any correspondence to classical models allowing for DB solutions? The second one is: how is

\footnotetext{
${ }^{4}$ See also 62.
} 
the DB solution obtained in a classical limit of a QM model allowing for quantum breathers? We will address both questions in the following.

\section{A. Weakly interacting oscillators}

Let us first discuss a system of weakly interacting oscillators given e.g. by (3.1). We can always choose a local basis given by the noninteracting system $\Phi(z)=0$. In this case we can formally solve the one-site problem of a particle moving in the potential $V(z)$. Denote the eigenvalues of the corresponding one-site Hamiltonian with $\epsilon_{n}$ with $n$ being the quantum number.

If the potential $V(z)$ is harmonic we find an equidistant eigenvalue spectrum e.g. $\epsilon_{n}=n$. Then still having $\Phi(z)=0$ the eigenvalues $E_{n}$ of the system of $N$ oscillators are given by $E_{n}=n$ where $n$ is defining the number of bosons excited. The linearity of $\epsilon_{n}$ in $n$ implies that these bosons are noninteracting (do not mix this interaction with the lattice interaction $\Phi(z))$. Consequently the degeneracies of $E_{n}$ are larger than $N$ except for $n=0,1: E_{0}$ is nondegenerate, $E_{1}$ is $N$-fold degenerate, $E_{2}$ is $N(N+1) / 2$-fold degenerate, $E_{3}$ is $N\left(N^{2}+2\right) / 3$-fold degenerate etc. Switching the interaction $\Phi(z)$ on we can expect all these degeneracies to be lifted. However except for the one boson band $n=1$ all other bands will consist of many more than $N$ states. Indeed we do not expect quantum breather bands for a harmonic function $\Phi(z)$.

Assume now that the potential $V(z)$ is anharmonic. Then the one-site eigenvalue spectrum $\epsilon_{n}$ is not equidistant in $n$ anymore. This change has consequencies for the eigenvalue spectrum of the system of $N$ noninteracting oscillators. Apart from changes in the eigenvalues the degeneracies are drastically reduced. The old eigenvalue $E_{2}$ splits now into two eigenvalues $-\epsilon_{2}$ with two bosons 5 on one lattice site (exactly $N$ states) and $2 \epsilon_{1}$ with two bosons on different lattice sites and $N(N-1) / 2$-fold degeneracy. The relative position of the two eigenvalues depends on the type of anharmonicity in $V(z)$. The old eigenvalue $E_{3}$ splits into three eigenvalues - $\epsilon_{3}$ with three bosons on one lattice site (exactly $N$ states), $\left(\epsilon_{2}+\epsilon_{1}\right)$ with two bosons on one lattice site and one boson on a separate lattice site $(N(N-1)$-fold degenerate) and $3 \epsilon_{1}$ with all three bosons on different lattice sites and $N(N-1)(N-2) / 3$-fold degeneracy. Thus the anharmonicity in $V(z)$ creates an infinity of states with $m$ bosons on one lattice site, which are exactly $N$-fold degenerate. We expect these states to be quantum breather states. The subsequent increase of $\Phi(z)$ will then lead to a finite band width of these breather bands with preserving the particle-like nature of the bound states.

These expectations (for nonvanishing interactions) have not been proven yet for a general case. One of the reasons is that the noninteracting case conserves the number of bosons, but the interacting case in general does not.

From the above it follows that quantum breather bands are likely to exist for systems with an antiintegrability limit $\Phi(z)=0$. The necessary condition is the anharmonicity of $V(z)$, which is also the necessary condition of finding classical DB solutions. Thus in this particular limit we expect a strong correspondence between the existence of classical DB solutions and the existence of quantum breather bands. Increasing the interaction $\Phi(z)$ the quantum bands broaden, and eventually overlap (hybridize) with other bands. Further increase of the interaction may or may not destroy the particle-like properties of the bound states. The correspondence between loosing/keeping quantum breather bands and classical DB solutions is a field yet to be explored.

A numerical analysis of quantum breather bands has been recently performed by Bishop et al [191]. The systems were one-dimensional, with a total number of lattice sites $N=4,8$. For $N=4$ seventeen states per site were taken into account, and six states per site for the case $N=8$. Low lying quantum breather bands were found (note that these bands consist of 4 or 8 states only). The particle-like properties of the breather states were successfully probed with the help of correlation functions. The smallness of the considered systems is due to computational limitations. Another recent computation of quantum breather states has been performed by Schofield eta al [158]. These authors aim at the understanding of local bond excitations in molecules and consider a system of six Morse oscillators coupled through nearest neighbour couplings on a ring (i.e. with periodic boundary conditions). Instead of calculating correlation functions Schofield et al calculate survival probabilities of local excitations and observe for weak coupling a bound state with approximately five bosons excited initially on a single oscillator.

\footnotetext{
${ }^{5}$ Note that here we are using the notion of a boson loosely to paraphrase the number state - a product state with each site excited to the $n$-th level ( $\mathrm{n}$ bosons at that site).
} 


\section{B. Systems with boson number conservation}

Much more is known for finite systems with an additional integral of motion (besides the energy) - the total number of bosons [102], [68], 159], [19]. This is so because the infinite dimensional Hilbert space separates then into an infinite set of finite dimensional noninteracting subspaces. Each subspace contains only states with the same total number of bosons. Consequently one deals now with the problem of diagonalizing each subspace separately, which requires diagonalizing only a finite dimensional matrix as opposed to an infinite dimensional one. As a result physically we have to solve only the problem of a finite number of interacting particles.

Let us consider the DNLS as a reference system. The classical equations are given in (2.6). The quantum problem is defined by replacing the complex variables $\psi_{l}$ and $\psi_{l}^{*}$ by an annihilation operator $a_{l}$ and a creation operator $a_{l}^{+}$ with boson commutation relation $\left[a_{l}, a_{l}^{+}\right]=1$ and choosing the Hamiltonian

$$
H=\kappa \sum_{l}\left(a_{l} a_{l}^{+}\right)^{2}+\sum_{l}\left(a_{l} a_{l+1}^{+}+a_{l}^{+} a_{l+1}\right) .
$$

Note that the second sum in (17.1) plays the role of a kinetic energy.

Bound states exist because of the boson number conservation. Several bosons initially close to each other can not separate because that would violate energy conservation. The existence of bound states for both attracting $(\kappa<0)$ or repulsive $(\kappa>0)$ boson-boson interaction is due to the discreteness of the system. The discreteness limits the kinetic energy from above, and thus even repelling bosons can form bound states.

\section{Two bosons}

Eilbeck et al 64] (see also [159]) have extensively considered the case of two bosons in one-dimensional lattices including the quantum DNLS (17.1), in which they found bound states. A typical result is shown in Fig. 14 where the band of bound states is well separated from the continuum of unbounded states. In the case of the quantized DNLS we can immediately find one bound state for $d$-dimensional lattices without frustration. This bound state is constructed out of a linear combination of local states with two bosons on one site. The prefactors in the linear combination have all the same absolute value and opposite signs for interacting sites. Note that this particular bound state does not contain any states with the two bosons separated! This state corresponds to the $k= \pm 50$ bound states in Fig. 14 . Since the two boson problem is essentially a two-body problem, it is particularly evident that bound states can exist.

\section{Many bosons}

Less is known for the case of more than two bosons. The only system studied extensively is the DNLS with two sites (dimer). Note that the discrete translational symmetry is here reduced to a mere permutational symmetry, i.e. the Hamiltonian is invariant under permutations of the two different site labels. This system is integrable due to the existence of two integrals of motion (energy and boson number). The classical version can be completely solved. Bernstein et al [19], [20] and Aubry et al [15] have studied the expected splitting of degenerate pairs of eigenvalues in the quantum system. The results demonstrate that there is a one-to-one correspondence between the existence of classical orbits which are not invariant under permutational symmetry, and the existence of exponentially small splittings of eigenvalues of corresponding eigenstates in the quantum problem. Aubry et al [15] have moreover demonstrated that the existence of a bifurcation of periodic orbits and the existence of a separatrix in the classical model is clearly reflected in the eigenvalue spectrum of the quantum model. Perturbation theories for the splittings of the corner states in [19] and for all states in [15] have been successfully applied.

Practically nothing is known for systems with many lattice sites and many bosons. Usage of the perturbation theory initially applied to the dimer suggests exponentially small bandwidths for quantum breather bands with large boson numbers [19], 13].

\section{Open questions}

So far we lack a proof for the existence of bound states. An exception are systems with boson number conservation and two bosons. We think that it should be possible to construct a rigorous proof for a general case of interacting oscillators. The analytical continuation of eigenstates and eigenenergies is easy, because in a linear eigenvalue problem 
there is no room for bifurcations (i.e. the number of eigenstates is fixed). The nontrivial part of a proof must show that certain properties of eigenstates can be continued, such as the property of an eigenstate to be a bound state of many bosons. Starting with zero interaction (antiintegrability limit) the continuation of low-lying bound states (i.e. where only a few bosons are involved) should be possible, because there is a finite distance to eigenvalues of other states.

A much harder problem is the description of many-boson bound states, because even in the limit of zero interaction of coupled oscillators there are plenty of other states with nearly the same eigenvalues. So one has to focus on why the overlap with those states is negligible small.

Further numerical diagonalizations following the promising results of Bishop et al [191] will be of help in studying the properties of bound states. Especially it is interesting to investigate the fate of states which in the limit of zero interaction between oscillators correspond to several groups of bosons (e.g. three bosons on one site and two bosons on another site). These states could correspond to classical multi-site breather solutions (see [137]).

\section{POSSIBLE EXPERIMENTAL REALIZATIONS}

In this section we will briefly discuss the experimental situations where discrete breathers can be expected to exist. The shortness of this section is not due to expected limitations of applicability, but rather due to the fact that only few things are known yet.

One possible situation to observe discrete breathers seems to be lattice dynamics of crystals. Anharmonicity is well known to exist and to contribute to several phenomena, e.g. heat expansion, structural phase transitions etc. We think that molecular crystals are particularly promising candidates. Especially molecular crystals and long molecules with intramolecular interactions being large compared to intermolecular interactions appear to be similar to the discussed systems of weakly interacting oscillators. Fillaux et al [69], [71], [72], [73], [70] are e.g. studying the inelastic neutron scattering by the molecular crystal 4-methyl-pyridine and observe quantum bound states of phonons. Breathers in polyacetylene were studied in [25], [26] and breathers in conjugated polymers in [147]. The problem of single bond excitation in molecules is a field of activity for itself. Many of the concepts recently emerging there can be unified with the concept of bound states in anharmonic lattices [185], 135.

Sievers and Page have reviewed the interrelation between discrete breathers and impurity modes in alkali halides 162 .

Similar effects can be expected for ferromagnetic and antiferromagnetic systems [33], [175]. We expect bound states of spin excitations to exist. These solutions will be quantum counterparts of classical spin breathers which are similar to the breather solutions of the DNLS.

The dynamics of coupled arrays of Josephson junctions is described with the help of a discretized sine Gordon equation, which is system (3.1) with $\Phi(z) \sim z^{2}$ and $V(z) \sim \cos z$. In addition there are damping terms. To excite any stationary solution an external driving is required. This is realized by applying an external current. Orlando et al 192. have measured the resulting voltage. By applying an ac driving (ac current) we expect discrete breathers to be excited. However their impact will be the creation of local fluctuations of the voltage, in contrast to a dc voltage which is typically measured.

Recent results on photonic band gap materials are of interest with respect to breathers. Photonic band gap materials are systems with a spatially periodic modulation of dielectric properties. Typically the wavelength is of the order of micrometers (visible light). Theoretical calculations suggest that Maxwell's equations if solved in such a medium give rise to allowed frequency bands of travelling light as well as to forbidden frequency gaps. Although there are some profound differences to the problem of electrons in a lattice (Schroedinger's equation) due to some symmetries, the idea is essentially that what works for electrons should also work for photons. The interested reader will find details in the reviews of Pendry [145], John [113] and Joannopoulos 112. All calculations that have been done are using linear optics. Consequently the solutions are always travelling light waves. The optical periodicity of the medium causes gaps to occur in the dispersion relation of light. There has been much discussion that the addition of defects opens the possibility of localizing light, and even guiding light along defined channels. Our point is that if media with optical nonlinearities [164] are used, then localization of light can appear even without adding defects. This can be of interest because no permanent changes to the material have to be done (as in the case of defects). The reason is that the dispersion relation of light in a homogeneous medium is $\omega \sim q$. Spatially periodic modulations yield gaps which are equidistant in $q$ and thus also equidistant in $\omega$. Thus we can fulfill the nonresonance condition for localized time-periodic solutions! For one-dimensional systems Sanchez et al 155 have demonstrated the existence of breathers.

Breathers have been observed in electrical lattices 141. We mention the demonstration of discrete breathers in macroscopic models of pendula with magnetic dipole-dipole interaction [153]. Discrete breathers can be easily observed 
in these systems provided the friction is not too strong. These systems are extremely easy to make, and can be a nice tool for demonstration.

Finally we discuss some properties of discrete breathers that might be important in designing experiments. The energy thresholds of discrete breathers in two- and three-dimensional lattices can be used - if the supplied energy is below the threshold, no breathers can be excited, and thus the energy is dissipated on a certain time scale due to phonons. If the supplied energy is above the threshold, discrete breathers are formed, and the decay is now defined by the lifetime of the discrete breather (which can be orders of magnitude larger than the typical phonon mediated decay time).

Another important feature is the scattering of plane waves by discrete breathers, which leads to strong reflection in the presence of a breather. Especially in one-dimensional systems one can probe the existence or nonexistence of a discrete breather by testing the transmittion properties of the system with respect to plane waves. A similarly interesting event is the possibility of trapped electron-breather states.

Moving discrete breathers can be of practical interest too. However we need to know more about movability in first place.

\section{CONCLUSIONS}

\section{A. Summary}

In this report we discussed existence and properties of discrete breathers. These solutions of Hamiltonian lattices are time-periodic and exponentially localized.

Discrete breathers are generic and structurally stable solutions because the neccessary nonresonance condition is easily fulfilled for a lattice. This condition requires the DB frequency as well as all of its multiples to not resonate with the linear spectrum of the system. Nonresonance is easy to achieve because the linear spectrum of a Hamiltonain lattice is bounded.

The nonresonance condition explains why breather solutions are nongeneric and structurally unstable in the opposing case of Hamiltonian fields.

Numerical studies show that long-lived localized excitations typically created in numerical experiments show up with periodic or quasiperiodic temporal behaviour. The periodic objects are discrete breathers. The quasiperiodic objects are phase space perturbed discrete breathers. Numerical studies suggest that quasiperiodic objects radiate energy in the form of small-amplitude waves and thus are not real solutions. Indeed the nonresonance condition is shown to be never fulfilled for the generic case of a quasiperiodic solution, although nongeneric exceptions can apply.

Discrete breathers are periodic orbits. For generic Hamiltonian systems periodic orbits occur in one-parameter families, and so do discrete breathers. The parameter describing the family can be the amplitude of a breather, or its frequency, or any other meaningful observable.

The spatial decay of a discrete breather is typically exponential. A Fourier series representation of the time-periodic discrete breather leads to Fourier number dependent exponents of the spatial decay. A linear theory can account for these exponents by using the frequency of the breather as an input parameter. In some cases nonlinear corrections apply, still allowing for a calculation of the exponents. Numerical calculations underpin the findings.

The decay of the Fourier components with increasing Fourier number is obtained. The essential finding are power laws with exponents depending on the lattice site.

Existence proofs of discrete breathers are reviewed for systems of weakly interacting oscillators and Fermi-PastaUlam-like systems. We discussed the antiintegrability approach of Aubry.

Several numerical methods to obtain discrete breather solutions are reviewed, and their advantages and weak points are discussed.

We discuss structural and dynamical stability of discrete breathers. Our essential finding is that discrete breathers are structurally stable, and typically can be dynamically stable (note that discrete breathers can as easily be also dynamically unstable).

The movability of discrete breathers is discussed at length, illuminating the conceptual problems. The main result is that a Peierls-Nabarro barrier can not be introduced in the way it had been done for moving lattice kinks.

The appearance of discrete breathers through bifurcations of band edge plane waves is discussed. The bifucation analysis of band edge plane waves for finite systems is demonstrated, and the results are used to predict the existence or nonexistence of discrete breathers for systems without actually solving for the discrete breather. Instead the band edge plane wave stability is analyzed.

The effect of lattice dimension on discrete breathers is discussed. Discrete breathers are not limited to certain dimensions of the lattice. However the lattice dimension has strong impact on the energy properties of discrete 
breathers. For lattice dimensions $d \geq 2$ the energy of discrete breathers has nonzero lower bounds, leading to an activation energy of discrete breathers.

We discuss the effect of discrete symmetries on discrete breathers. Discrete breather solutions are not invariant under discrete translations. However they can be invariant under other discrete symmetries (provided the Hamiltonain is). This has practical consequencies, since the number of independent Fourier coefficients is reduced with every additional discrete symmetry.

We refer the reader to section XIII for a complete conceptual summary of the first twelve sections.

We review phonon and electron scattering by discrete breathers. Discrete breathers turn out to be strong scatterers of phonons and electrons and will thus be of interest whenever heat or charge transport is considered.

Plane wave trapping is especially important in the case of electrons. Bound electron-discrete breather states exist, and can be of conceptual interest in many areas of solid state theory.

The connection between disorder and nonlinearity with respect to discrete breathers is briefly discussed. The main point is that discrete breathers could be directly (or through bifurcations) connected to the well-known impurity states of linear systems.

The correspondence between classical discrete breathers and quantum bound states (quantum breathers) is discussed and some questions in this new area are raised.

Finally we briefly mention possible experimental realizations of discrete breathers.

\section{B. Unsolved problems}

We are unable to list all unsolved problems with respect to discrete breathers. However in the following we list problems that arose in this report.

In connection with the discussion of field equations in the introduction we expect Boyd's nanopteron [34] to be of further interest. Discrete breathers will most probably become partially delocalized nanopterons once a higher harmonics of the breather frequency hits the linear spectrum. In this sense there can exist a natural connection between discrete breathers and nanopterons or breathers in some field equations. Discrete breathers as well as breathers of field equations are solutions of finite energy. Nanopterons are solutions of infinite energy. Staying within the class of finite energy solutions there is most probably no link between discrete breathers and breathers of field equations. However including solutions with infinite energy the missing link might be recovered.

The spatial decay of discrete breathers in systems with acoustic spectra has to be studied. The lack of displacement reversal symmetry leads to the excitation of even Fourier components. The dc component corresponds to zero frequency and will thus resonate with the lower bound of the acoustic band of the linear spectrum. It is not clear (although we presented some arguments) how this resonance is reflected in the localization properties of discrete breathers, especially for two- and three-dimensional lattices.

A problem related to the above one is the importance of polarization vectors for the spatial decay. Suppose a lattice has more than one degrees of freedom per unit cell. Then scenarios are possible where optical and acoustic spectra coexist. What happens if a breather is assumed to bifurcate off a band edge plane wave of the optical spectrum, provided it contains a dc component due to lack of symmetry? Will this dc component be affected by the resonance with the acoustic spectrum? What is again the influence of lattice dimension on the findings?

Concerning the dynamical stability the radiation of perturbed breather solutions (quasiperiodic objects) has to be quantified. Also the apparent differences in the nonlinear stability analysis depending on the choosen perturbed solutions need further investigation.

Do moving breather solutions exist or not? Thus far we are lacking proofs and complete numerical investigations.

What about the possibility of thermally exciting discrete breathers? Dauxois et al [50, Burlakov et al [36] and Flach and Siewert 86] report on numerical evidence that for some models finite temperature simulations show the existence of breather-like objects with finite lifetime (the lifetime in the cited cases was considerably larger than the breathers inverse frequency, thus the identification is meaningful). Anomalous slow relaxations in the time-dependent correlator of energy density fluctuations has been connected with the existence of breathers [85], [183]. How is the observed lifetime connected to our analysis of dynamical stability of discrete breathers?

Further studies are required to clarify the domain of existence of discrete breathers. Adding a friction will certainly make discrete breathers disappear. However addition of ac driving can actually phaselock discrete breathers [139], [151], 44]. For some results on phase-locked sG field breathers check [99], 115].

Much more is needed to be known about the scattering properties, bound states, quantum breathers. Recent results by MacKay et al 138 and Pikovsky et al 148 use the breather concept for finding corresponding solutions for networks of weakly coupled bistable units and weakly coupled symplectic maps respectively. 
And finally we are waiting for serious experimental evidence that discrete breathers are detected. This hope is based on the fact that discrete breathers are generic solutions of nonlinear Hamiltonian lattices in one, two, three etc dimensions. In some sense quantum bound states of bosons and fermions can be regarded as quantum breathers.

\section{Some closing words}

We reviewed a new area of research which is studying discrete breathers. The aim of the review was to give the reader both an overview of the achieved results, and to provide linking thoughts in order to embed the results into the general field of physics. Most certainly we forgot to appropriately mention several results, and for this we sincerely apologize ${ }^{6}$. At this place we want to thank many colleagues for sharing their views and discussing related problems with us. Especially important were discussions with S. Aubry, U. Bahr, L. Bernstein, O. Bang, D. Campbell, Th. Cretegny, C. Eilbeck, V. Fleurov, P. Fulde, K. Kladko, R. Livi, R. S. MacKay, E. Olbrich, T. Orlando, M. Peyrard, M. Wagner, and many others. This work was funded in parts by the Deutsche Forschungsgemeinschaft. Finally S.F. wants to thank Elja and Nora for the indescribable that helped so much.

\section{WHAT'S NEW?}

This section is added in print. It refers to the previous sections in this work and briefly mentions new results which appeared in the past months after the original work was submitted.

\section{NEWS IN IVD}

The spatial decay considered in chapter IVD was considered for lattices with nearest neighbour interaction. What matters however is the dispersion of the phonon band at the band edges. Since the spatial decay of the breather solution far from its center is given by the Green's function

$$
G_{\lambda}(n)=\int \frac{\cos (k n)}{\lambda^{2}+\Omega_{k}^{2}-1} \mathrm{~d} k
$$

for large values of $n$, the fast oscillation of the numerator allows for an expansion of the denominator around the band edges. If the dispersion is quadratic at the band edge, the poles of the Green's function are located on the imaginary axis, and exponential decay in space follows. If however the interaction is long-range, the dispersion relation near the band edge will change, e.g. for an interaction $1 / r^{s}$ in one-dimensional lattices Gaididei et al [96] find

$$
\Omega_{k}^{2}=1+C \zeta(s-2) k^{2}(s>3), \Omega_{k}^{2}=1+2 C a(s) k^{s-1}(1<s<3),
$$

with $\zeta(s)$ being the Riemann Zeta function. Consequently in this example

$$
G_{\lambda}(n) \sim \mathrm{e}^{-\lambda n}(s>3), G_{\lambda}(n) \sim n^{-s}(1<s<3) .
$$

The algebraic decay in space found for $s<3$ follows from the fact that the poles of the Green's function leave the imaginary axis starting with $s=3$, caused in turn by the change in the dispersion relation at the band edge.

\section{NEWS IN IV G}

The heuristically predicted algebraic decay of the dc-component has been recently confirmed for $d=1$ by an analytical proof of the existence of acoustic breathers [136]. The existence of the static kink structure predicted in [V G and found numerically is proven.

\footnotetext{
${ }^{6} \mathrm{An}$ update of DB related publications is located at the web page: http://www.mpipks-dresden.mpg.de/ flach/breather.DIR/db.bib
} 


\section{NEWS IN $\mathrm{VA}$}

As already mentioned, Livi, MacKay and Spicci [136 proved the existence of acoustic breathers, i.e. breathers in the absence of on-site potentials. A diatomic chain was considered. The limit of infinite heavy masses yields a starting point for the continuation of spatially localized periodic orbits. The general problem is that the Newton operator is not invertible if considered in the original variables. This happens because the phonon spectrum degenerates into two values in the considered limit - one optical value (nonzero frequency) and one value zero. This zero is removed in [136] by considering new variables. The continuation is only possible if a corresponding strain field of dc distortions is imposed. This distortion turns out to be of a simple kink structure.

\section{NEWS IN IX}

A recent work by Chen et al 42 attempts to create moving breathers by considering stable stationary breathers with a localized eigenstate of the corresponding Floquet matrix (orthogonal to the breather family). Perturbations in the direction of the localized eigenstate will cross the movability separatrix as the amplitude of the perturbation exceeds some critical value (this was already discussed in [88]). Chen et al find trajectories similar to moving breathers over long periods of time.

\section{NEWS IN XI}

As already mentioned, the spatial decay of the breather is essentially governed by the phonon dispersion at the band edges. In order to obtain energy thresholds for breathers, exponential decay in space is needed, with exponents depending on the breather parameter. Thus the results from XI can be extended to systems with nonlocal interactions, provided the quadratic dispersion at the band edges holds.

\section{NEWS IN XVII B 2}

A recent study of a trimer [81] considered the fate of tunneling pairs of the quantum dimer when the phase space of the classical problem becomes mixed. Tunneling pairs survive avoided crossings with single states and other tunneling pairs, and get finally destroyed when the classical phase space which supports these states turns chaotic. An application of the results to the concept of doorway states for local bond excitations in molecules confirms that simplistic arguments like the lifetime of a state being proportional to the density of nearby lying eigenstates can be very misleading.

\section{NEWS IN XVIII}

Bound states of phonons have been measured in the following systems: Hydrogen on $\operatorname{Si}(111)$ surface [101], crystal $\mathrm{CO}_{2}$ [22], $\mathrm{CO}$ on $\mathrm{Ru}(001)$ surface [11]. Corresponding measurements for small molecules (benzene, naphtalene, anthracene) are known for a long time [166].

A recent work by Floria et al 91 demonstrates theoretically that in an anisotropic ladder realization of Josephson junctions rotobreathers can be excited, which would lead to the generation of a dc voltage and thus measurable.

Kisilev et al have calculated breathers in ionic crystals [123], and Rössler et al proposed optically controlled generation of breathers 152 . 
[1] M. J. Ablowitz and J. F. Ladik. Nonlinear differential-difference equations and fourier analysis. J. Math. Phys., 17:1011, 1976.

[2] E. Akkermans, G. Montambaux, J. L. Pichard, and J. Zinn-Justin, editors. Mesoscopic Quantum Physics. Elsevier Amsterdam, 1995.

[3] C. Albanese and J. Frohlich. Periodic solutions of some infinite-dimensional hamiltonians associated with nonlinear partial differential equations i. Comm. Math. Phys., 116:475, 1988.

[4] C. Albanese and J. Frohlich. Perturbation theory for periodic orbits in a class of infinite dimensional hamiltonian systems. Comm. Math. Phys., 138:193, 1991.

[5] C. Albanese, J. Frohlich, and T. Spencer. Periodic solutions of some infinite-dimensional hamiltonians associated with nonlinear partial differential equations ii. Comm. Math. Phys., 119:677, 1988.

[6] L. M. Alonso, E. M. Reus, and E. O. Moreno. Breathers in 2+1 dimensions. Phys. Lett. A, 159:384, 1991.

[7] M. Aoki. Self-localized mode in a diatomic nonlinear lattice. J. Phys. Soc. Japan, 61:3024, 1992.

[8] M. Aoki and S. Takeno. Stationary anharmonic gap modes in the diatomic toda chain. J. Phys. Soc. Japan, 64:809, 1995.

[9] M. Aoki, S. Takeno, and A. J. Sievers. Stationary anharmonic gap modes in a one-dimensional diatomic lattice with quartic anharmonicity. J. Phys. Soc. Japan, 62:4295, 1993.

[10] V. I. Arnold. Mathematical Methods of Classical Mechanics. Springer-Verlag New York, 1989.

[11] V. I. Arnold. Ordinary Differential Equations. Springer Berlin, 1992.

[12] S. Aubry. The concept of anti-integrability applied to dynamical systems and to structural and electronic models in condensed matter physics. Physica, D71:196, 1994.

[13] S. Aubry. Breathers in nonlinear lattices: Existence, linear stability and quantization. Physica D, in print, 1997.

[14] S. Aubry and G. Abramovici. Chaotic trajectories in the standard map. the concept of anti-integrability. Physica D, 43:199, 1990.

[15] S. Aubry, S. Flach, K. Kladko, and E. Olbrich. Manifestation of classical bifurcation in the spectrum of the integrable quantum dimer. Phys. Rev. Lett., 76:1607, 1996.

[16] D. Bambusi. Exponential stability of breathers in hamiltonian networks of weakly coupled oscillators. Nonlinearity, 9:433, 1996.

[17] O. Bang and M. Peyrard. Higher order breathers solutions to a discrete nonlinear klein-gordon model. Physica, D81:9, 1995.

[18] O. Bang and M. Peyrard. Generation of high-energy localized vibrational modes in nonlinear klein-gordon lattices. Phys. Rev., E53:4143, 1996.

[19] L. Bernstein, J. C. Eilbeck, and A. C. Scott. The quantum theory of local modes in a coupled system of nonlinear oscillators. Nonlinearity, 3:293, 1990.

[20] L. J. Bernstein. Quantizing a self-trapping equation. Physica D, 68:174, 1993.

[21] S. R. Bickham, S. A. Kisilev, and A. J. Sievers. Stationary and moving intrinsic localized modes in one-dimensional monoatomic lattices with cubic and quartic anharmonicity. Phys. Rev., B47:14206, 1993.

[22] R. Bini, P. R. Salvi, V. Schettino and H. J. Jodl. The spectroscopy and relaxation dynamics of three-phonon bound states in crystal $\mathrm{CO}_{2}$. J. Chem. Phys., 98:164, 1993.

[23] B. Birnir. Nonexistence of periodic solutions to hyperbolic partial differential equations. In M. Gyllenberg and L. E. Persson, editors, Analysis, Algebra, and Computers in Mathematical Research, page 43. Marcel Dekker, Inc. New York, 1994.

[24] B. Birnir. Qualitative analysis of radiating breathers. Comm. Pure Appl. Math., XLVII:103, 1994.

[25] A. R. Bishop, D. C. Campbell, P. S. Lomdahl, B. Horowitz, and S. R. Phillpot. Breathers and photoinduced absorption in polyacetylene. Phys. Rev. Lett., 52:671, 1984.

[26] S. Block and H. W. Streitwolf. Nucleation of optically excited solitrons and breathers in trans-polyacetylene. J. Phys.: Cond. Matter, 8:889, 1996.

[27] R. Boesch and M. Peyrard. Discreteness effects on a sine-gordon breather. Phys. Rev., B43:8491, 1991.

[28] R. Boesch, P. Stancioff, and C. R. Willis. Hamiltonian equations for multiple-collective-variable theories of nonlinear klein-gordon equations: A projection-operator approach. Phys. Rev., B38:6713, 1988.

[29] R. Boesch and C. R. Willis. Exact determination of the peierls-nabarro frequency. Phys. Rev., B39:361, 1989.

[30] R. Boesch and C. R. Willis. Removal of singularities from collective-variable theory by incorporating relativistic invariance. Phys. Rev., A45:5422, 1992.

[31] D. Bonart, A. P. Mayer, and U. Schröder. Anharmonic localized surface vibrations in a scalar model. Phys. Rev., B51:13739, 1995.

[32] D. Bonart, A. P. Mayer, and U. Schröder. Intrinsic localized anharmonic modes at crystal edges. Phys. Rev. Lett., 75:870, 1995. 
[33] J. P. Boucher, F. Mezei, L. P. Regnault, and J. P. Renard. Diffusion of solitons in the antiferromagnetic chains of $\left(c_{3}\right)_{4} n_{m n c l}$ : A study by neutron spin echo. Phys. Rev. Lett., 55:1778, 1985.

[34] J. P. Boyd. A numerical calculation of a weakly non-local solitary wave: the cap phi 4 breather. Nonlinearity, 3:177, 1990.

[35] V. M. Burlakov and S. A. Kisilev. Molecular-dynamics simulation of the decay kinetics of uniform excitation of an anharmonic 1d chain. JETP, 72:854, 1991.

[36] V. M. Burlakov, S. A. Kisilev, and V. N. Pyrkov. Computer simulation of intrinsic localized modes in one-dimensional and two-dimensional anharmonic lattices. Phys. Rev. B, 42:4921, 1990.

[37] V. M. Burlakov, S. A. Kisilev, and V. N. Pyrkov. Computer simulations of intrinsic localized modes in 1-d anharmonic lattices. Solid State Comm., 74:327, 1990.

[38] V. M. Burlakov, S. A. Kisilev, and V. I. Rupasov. Localized excitations of uniform anharmonic lattices. JETP Lett., $51: 544,1990$.

[39] V. M. Burlakov, S. A. Kisilev, and V. I. Rupasov. Localized vibrations of homogeneous anharmonic chains. Phys. Lett., A147:130, 1990.

[40] D. Cai, A. R. Bishop, and N. Grønbech-Jensen. Spatially localized, temporally quasiperiodic, discrete nonlinear excitations. Phys. Rev., E52:R5784, 1995.

[41] D. K. Campbell and M. Peyrard. in: CHAOS - Soviet American Perspectives on Nonlinear Science, ed. by D. K. Campbell. American Institute of Physics New York, 1990.

[42] D. Chen, S. Aubry and G. P. Tsironis. Breather mobility in discrete $\phi^{4}$ nonlinear lattices. Phys. Rev. Lett., 77:4776, 1996.

[43] B. V. Chirikov. A universal instability of many-dimensional oscillator systems. Physics Reports, 52:263, 1979.

[44] P. L. Christiansen, Yu, B. Gaididei, M. Johansson and K. O. Rasmussen. Breatherlike excitations in discrete lattices with noise and nonlinear damping. Phys. Rev. B, 55:5759, 1997.

[45] O. A. Chubykalo and Y. S. Kivshar. Kink-profile vibrational modes in one-dimensional nonlinear lattices. Phys. Lett., A178:123, 1993.

[46] O. A. Chubykalo, A. S. Kovalev, and O. V. Usatenko. Stability of intrinsic localied modes in anharmonic 1-d lattices. Phys. Lett. A, 178:129, 1993.

[47] C. Claude, Yu. S. Kivshar, O. Kluth, and K. H. Spatschek. Moving localized modes in nonlinear lattices. Phys. Rev., B47:14228, 1993.

[48] T. Cretegny, S. Aubry and S. Flach. in preparation, 1997.

[49] R. F. Dashen, B. Hasslacher, and A. Neveu. Nonperturbative methods and extended-hadron models in field theory ii. Phys. Rev. D, 10:4130, 1974.

[50] T. Dauxois, M. Peyrard, and A. R. Bishop. Dynamics and thermodynamics of a nonlinear model for dna denaturation. Phys. Rev. E, 47:684, 1993.

[51] T. Dauxois, M. Peyrard, and C. R. Willis. Localized breather-like solution in a discrete klein-gordon model and application to dna. Physica D, 57:267, 1992.

[52] T. Dauxois, M. Peyrard, and C. R. Willis. Discreteness effects on the formation and propagation of breathers in nonlinear klein-gordon equations. Phys. Rev., E48:4768, 1993.

[53] A. S. Davydov. Quantum Mechanics. Pergamon Press, New York, 1976.

[54] J. Denzler. Nonpersistence of breather families for the perturbed sine gordon equation. Commun. Math. Phys., 158:397, 1993.

[55] J. Denzler. Second order nonpersistence of the sine-gordon breather under an exceptional perturbation. Annales de l'institut henri poincare, 12:201, 1995.

[56] J. Deudonne. Foundations of Modern Analysis. Academic New York, 1969.

[57] P. Tchofo Dinda, R. Boesch, E. Coquet, and C. R. Willis. Discreteness effects on the double-quadratic kink. Phys. Rev., B46:3311, 1992.

[58] R. K. Dodd, J. C. Eilbeck, J. D. Gibbon, and H. C. Morris. Solitons and Nonlinear Wave Equations. Academic Press New York, 1982.

[59] D. B. Duncan, J. C. Eilbeck, H. Feddersen, and J. A. D. Wattis. Solitons on lattices. Physica D, 68:1, 1993.

[60] R. Dusi, G. Viliani and M. Wagner. Breathing self-localized solitons in the quartic Fermi-Pasta-Ulam chain. Phys. Rev. $B, 54: 9809,1996$.

[61] R. Dusi and M. Wagner. A gauss procedure for the construction of localized solitons in anharmonic chains. Physica, B219-220:393, 1996.

[62] W. Ebeling and M. Jenssen. Trapping and fusion of solitons in a nonuniform toda lattice. Physica D, 32:183, 1988.

[63] E. N. Economou. Green's Functions in Quantum Physics. Springer-Verlag Heidelberg, 1983.

[64] J. C. Eilbeck, H. Gilhøj, and A. C. Scott. Soliton bands in anharmonic quantum lattices. Phys. Lett. A, $172: 229,1993$.

[65] J. C. Eilbeck, P. S. Lomdahl, and A. C. Scott. The discrete self-trapping equation. Physica D, 16:318, 1985.

[66] V. M. Eleonskii, N. E. Kulagin, N. S. Novozhilova, and V. P. Shilin. Asymptotic expansions and qualitative analysis of finite-dimensional models in nonlinear field theory. Teor. Mat. Fiz., 60:395, 1984.

[67] S. Elytin, A.Maimistov, and E. Manykin. Numerical study of fast oscillating breathers in the self-induced transparency phenomenon. Phys. Lett. A, 142:493, 1989.

[68] V. Z. Enolskii, M. Salerno, A. C. Scott, and J. C. Eilbeck. There's more than one way to skin schrödinger's cat. Physica 
$D, 59: 1,1992$.

[69] F. Fillaux and C. J. Carlile. Inelastic-neutron-scattering study of methyl tunneling and the quantum sine-gordon breather in isotopic mixtures of 4-methyl-pyridine at low temperature. Phys. Rev. B, 42:5990, 1990.

[70] F. Fillaux, C. J. Carlile, and A. Inaba. Relaxation kinetics of the sine-gordon breather mode in 4-methyl-pyridine crystal at low temperature. Physica B, 213 - 214:646, 1995.

[71] F. Fillaux, C. J. Carlile, and G. J. Kearley. Inelastic-neutron-scattering study at low temperature of the quantum sinegordon breather in 4-methyl-pyridine with partially deuterated methyl groups. Phys. Rev. B, 44:12280, 1991.

[72] F. Fillaux, C. J. Carlile, and G. J. Kearley. Inelastic neutron scattering study at low temperature of the quantum sine-gordon breather in 4-methyl-pyridine with partially deuterated methyl groups. Physica B, 180 - 181:642, 1992.

[73] F. Fillaux, C. J. Carlile, and M. Prager. Inelastic neutron-scattering study of methyl tunneling and the quantum sinegordon breather mode in isotopic mixtures of 2,6-dimethyl-pyridine at low temperature. Physica B, 202:302, 1994.

[74] F. Fischer. Self-localized single-anharmonic vibrational modes in two-dimensional lattices. Ann. Physik, 2:296, 1993.

[75] F. Fischer. Three-particle quartic-power dynamics: an integrable model. Phys. Lett., A182:417, 1993.

[76] S. Flach. Conditions on the existence of localized excitations in nonlinear discrete systems. Phys. Rev. E, 50:3134, 1994.

[77] S. Flach. Existence of localized excitations in nonlinear hamiltonian lattices. Phys. Rev. E, 51:1503, 1995.

[78] S. Flach. Obtaining breathers in nonlinear hamiltonian lattices. Phys. Rev. E, 51:3579, 1995.

[79] S. Flach. Existence and properties of discrete breathers. In E. Alfinito, M. Boiti, L. Martina, and F. Pempinelli, editors, Nonlinear Physics Theory and Experiment, page 390. World Scientific Singapore, 1996.

[80] S. Flach. Tangent bifurcation of band edge plane waves, dynamical symmetry breaking and vibrational localization. Physica, D91:223, 1996.

[81] S. Flach and V. Fleurov. Tunneling in the nonintegrable trimer - a step towards quantum breathers. J. Phys.: Cond. Mat., accepted, 1997.

[82] S. Flach and K. Kladko. Interaction of discrete breathers with electrons in nonlinear lattices. Phys. Rev., B53:11531, 1996.

[83] S. Flach, K. Kladko, and R. S. MacKay. Energy thresholds of discrete breathers in one-, two- and three-dimensional lattices. Phys. Rev. Lett., 78:1207, 1997.

[84] S. Flach, K. Kladko, and C. R. Willis. Localized excitations in two-dimensional lattices. Phys. Rev. E, 50:2293, 1994.

[85] S. Flach and G. Mutschke. Slow relaxation and phase space properties of a conservative system with many degrees of freedom. Phys. Rev. E, 49:5018, 1994.

[86] S. Flach and J. Siewert. Fast and slow dynamics in the one-dimensional $\phi^{4}$ lattice model - a molecular dynamics study. Phys. Rev., B47:14910, 1993.

[87] S. Flach and C. R. Willis. Nonlinear localized excitations in a discrete klein-gordon model. Phys. Lett. A, 181:232, 1993.

[88] S. Flach and C. R. Willis. Movability of localized excitations in nonlinear discrete systems. Phys. Rev. Lett., 72:1777, 1994.

[89] S. Flach and C. R. Willis. in: Nonlinear Excitations in Biomolecules, ed. by M. Peyrard. Editions de Physique, SpringerVerlag, 1995.

[90] S. Flach, C. R. Willis, and E. Olbrich. Integrability and localized excitations in nonlinear discrete systems. Phys. Rev. E, 49:836, 1994.

[91] L. M. Floria, J. L. Marin, P. J. Martinez, F. Falo and S. Aubry. Intrinsic localization in the dynamics of a Josephsonjunction ladder. Europhys. Lett., 36:539, 1996.

[92] N. Flytzanis, S. Pnevmatikos, and M. Remoissenet. Kink, breather and assymmetric envelope or dark solitons in nonlinear chains: I. monoatomic chain. J. Phys. C: Solid State Phys., 18:4603, 1985.

[93] K. Forinash, M. Peyrard, and B. A. Malomed. Interaction of discrete breathers with impurity modes. Phys. Rev. E, 49:3400, 1994.

[94] J. Frohlich, T. Spencer, and C. E. Wayne. Localization in disordered nonlinear dynamical systems. J. Stat. Phys., 42:247, 1986.

[95] P. Fulde. Electron Correlations in Molecules and Solids. Springer Berlin, 1995.

[96] Yu. B. Gaididei, S. F. Mingaleev, P. L. Christiansen and K. Ø. Rasmussen. Effects of nonlocal dispersive interactions on self-trapping excitations. preprint, 1997.

[97] J. Geicke. Logarithmic decay of phi4 breathers of energy less than or equal to 1. Phys. Rev., E49:3539, 1994.

[98] J. Goldstone and R. Jackiw. Quantization of nonlinear waves. Phys. Rev. D, 11:1486, 1975.

[99] R. Grauer and Yu. S. Kivshar. Chaotic and phase-locked breather dynamics in the damped and parametrically driven sine-gordon equation. Phys. Rev. E, 48:4791, 1993.

[100] S. A. Gredeskul and Yu. S. Kivshar. Propagation and scattering of nonlinear waves in disordered systems. Physics Reports, 216:1, 1992.

[101] P. Guyot-Sionnest. Two-phonon bound state for the Hydrogen vibration on the H/Si(111) surface. Phys. Rev. Lett., 67:2323, 1991.

[102] M. H. Hays and A. C. Scott. Quantizing the discrete self-trapping equation. Phys. Lett. A, 188:21, 1994.

[103] D. Hennig, K. O. Rasmussen, H. Gabriel and A. Bülow. Solitonlike solutions of the generalized discrete nonlinear Schrödinger equation. Phys. Rev. E, 54:5788, 1996.

[104] D. Hennig, K. Ø. Rasmussen, G. P. Tsironis, and H. Gabriel. Breatherlike impurity modes in discrete nonlinear lattices. 
Phys. Rev. B, 52:R4628, 1995.

[105] M. Hisakado and M. Wadati. Dynamics of breather modes in a nonlinear model of dna with discontinuity. J. Phys. Soc. Japan, 64:1910, 1995.

[106] K. Hori. Wavelet analysis of anharmonic self-localized modes. J. Phys. Soc. Japan, 62:1819, 1993.

[107] K. Hori and S. Takeno. Low-frequency and high-frequency moving anharmonic localized modes in a one-dimensional lattice with quartic anharmonicity. J. Phys. Soc. Japan, 61:4263, 1992.

[108] K. Hori and S. Takeno. Moving self-localized modes for the displacement field in a one-dimensional lattice system with quartic anharmonicity. J. Phys. Soc. Japan, 61:2186, 1992.

[109] K. Hori and S. Takeno. Soliton scattering by an impurity on a nonlinear lattice. Phys. Lett., A169:355, 1992.

[110] G. Huang, Z. Shi, and Z. Xu. Asymmetric intrinsic localized modes in a homogeneous lattice with cubic and quartic anharmonicity. Phys. Rev., B47:14561, 1993.

[111] P. Jakob. Dynamics of the C-O stretch overtone vibration of CO/Ru(001). Phys. Rev. Lett., 77:4229, 1996.

[112] J. D. Joannopoulos, R. D. Meade, and J. N. Winn. Photonic Crystals. Princeton University Press Princeton, 1995.

[113] S. John. The localization of light. In C. M. Soukoulis, editor, Photonic Band Gaps and Localization, page 1993. Plenum New York, 1993.

[114] W. Jones and N. H. March. Theoretical Solid State Physics. Dover Publications New York, 1973.

[115] J. D. Kaup and A. Newell. Theory of nonlinear oscillating dipolar excitations in one-dimensional condensates. Phys. Rev. $B, 18: 5162,1978$.

[116] S. Kichenassamy. Breather solutions of the nonlinear wave equation. CPAM, 44:789, 1991.

[117] S. A. Kisilev. Stationary vibrational modes if a chain of particles interacting via an even order potential. Phys. Lett., A148:95, 1990.

[118] S. A. Kisilev, S. R. Bickham, and A. J. Sievers. Anharmonic gap modes in a perfect one-dimensional diatomic lattice for standard two-body nearest-neighbor potentials. Phys. Rev. B, 48:13508, 1993.

[119] S. A. Kisilev, S. R. Bickham, and A. J. Sievers. Anharmonic gap mode in a one-dimensional diatomic lattice with nearest-neighbor born-mayer-coulomb potentials and its interaction with a mass-defect impurity. Phys. Rev., B50:9135, 1994.

[120] S. A. Kisilev, S. R. Bickham, and A. J. Sievers. Anharmonic impurity modes in a 1-d lattice with two-body potentials. Phys. Lett., A184:255, 1994.

[121] S. A. Kisilev, S. R. Bickham, and A. J. Sievers. Localized anharmonic defect modes in a one-dimensional lattice with standard two-body nearest-neighbor potentials. Journal of Luminescence, 58:23, 1994.

[122] S. A. Kisilev and V. I. Rupasov. Stationary vibrational modes of a polyatomic chain of particles interacting via an even order potential. Phys. Lett., A148:355, 1990.

[123] S. A. Kisilev and A. J. Sievers. Generation of intrinsic vibrational gap modes in three-dimensional ionic crystals. Phys. Rev. B, 55:5755, 1997.

[124] T. Kitamura and S. Takeno. Solitons and bound states of the self-consistent potential by the boson transformation method. Phys. Lett., A172:184, 1992.

[125] Y. S. Kivshar and M. Peyrard. Modulational instabilities in discrete lattices. Phys. Rev., A46:3198, 1992.

[126] Yu. S. Kivshar. Intrinsic localized modes as solitons with compact support. Phys. Rev. E, 48:R43, 1993.

[127] Yu. S. Kivshar. Localized modes in a chain with nonlinear on-site potential. Phys. Lett., A173:172, 1993.

[128] Yu. S. Kivshar. Nonlinear impurity modes in a lattice. Phys. Rev. B, 47:11167, 1993.

[129] Yu. S. Kivshar and D. K. Campbell. Peierls-nabarro potential barrier for highly localized nonlinear modes. Phys. Rev. E, 48:3077, 1993.

[130] Yu. S. Kivshar and S. K. Turitsyn. Lattice solitons on a standing carrier wave. Phys. Lett. A, 171:344, 1992.

[131] A. M. Kosevich and A. S. Kovalev. Selflocalization of vibrations in a one-dimensional anharmonic chain. JETP, 67:1793, 1974.

[132] E. W. Laedke, O. Kluth, and K. H. Spatschek. Existence of solitary solutions in nonlinear chains. Phys. Rev. E, 54:4299, 1996.

[133] L. D. Landau and E. M. Lifshitz. Elastizitätstheorie, Lehrbuch der Theoretischen Physik VII. Akademie-Verlag Berlin, 1991.

[134] L. D. Landau and E. M. Lifshitz. Quantenmechanik, Lehrbuch der Theoretischen Physik III. Akademie-Verlag Berlin, 1991.

[135] K. K. Lehman, G. Scoles, and B. H. Pate. Intramolecular dynamics from eigenstate-resolved infrared spectra. Ann. Rev. Phys. Chem., 45:241, 1994.

[136] R. Livi, R. S. MacKay and M. Spicci. Breathers on a diatomic FPU chain. Nonlinearity, submitted, 1997.

[137] R. S. MacKay and S. Aubry. Proof of existence of breathers for time-reversible or hamiltonian networks of weakly coupled oscillators. Nonlinearity, 7:1623, 1994.

[138] R. S. MacKay and J. A. Sepulchre. Multistability in networks of weakly coupled bistable units. Warwick preprint, 44, 1994.

[139] B. A. Malomed. Damping and pumping of localized intrinsic modes in nonlinear dynamical lattices. Phys. Rev., B49:5962, 1994. 
[140] J. L. Marin and S. Aubry. Breathers in nonlinear lattices: numerical continuation from the anticontinuous limit. Nonlinearity, 9:1501, 1996.

[141] P. Marquie, J. M. Bilbaut, and M. Remoissenet. Observation of nonlinear localized modes in an electrical lattice. Phys. Rev., E51:6127, 1995.

[142] J. Carr and B. McLeod. Solitary waves on lattices. preprint, 1997.

[143] A. H. Nayfeh. Introduction to Perturbation Techniques. John Wiley and Sons, New York, 1993.

[144] A. Neuper, F. G. Mertens, and N. Flytzanis. Quasicontinuum approximation and iterative method for envelope solitons in anharmonic chains. Z. Phys., B95:397, 1994.

[145] J. B. Pendry. Calculating photonic band structure. J. Phys.: Condens. Matter, 8:1085, 1996.

[146] P. Perfetti. An infinite-dimensional extension of a poincare's result concerning the continuation of periodic orbits. preprint, 1996.

[147] S. R. Phillpot, A. R. Bishop, and B. Horovitz. Amplitude breathers in conjugated polymers. Phys. Rev. B, 40:1839, 1989.

[148] M. Abel, A. Pikovsky and S. Flach. in preparation, 1997.

[149] W. H. Press, B. P. Flannery, S. A. Teukolsky, and W. T. Vetterling. Numerical Recipes : the art of scientific computing. Cambridge University Press, 1986.

[150] M. Remoissenet. Low-amplitude breather and envelope solitons in quasi-one-dimensional physical models. Phys. Rev., B33:2386, 1986.

[151] T. Rössler and J. B. Page. Driven intrinsic localized modes and their stability in anharmonic lattices with realistic potentials. Physica, B219-220:387, 1996.

[152] T. Rössler and J. B. Page. Creation of intrinsic localized modes via control of anharmonic lattices. Phys. Rev. Lett., 78:1287, 1997.

[153] F. M. Russell, Y. Zolotaryuk, J. C. Eilbeck, and T. Dauxois. Moving breathers in a chain of magnetic pendulums. Phys. Rev. B, 55:6304, 1997.

[154] R. S. Sagdeev, D. A. Usikov, and G. M. Zaslavski. Nonlinear Physics: from the Pendulum to Turbulence and Chaos. Harwood Academic Publishers, 1988.

[155] A. Sanchez, R. Scharf, A. R. Bishop, and L. Vasquez. Sine-gordon breathers on spatially periodic potentials. Phys. Rev. A, 45:6031, 1992.

[156] K. W. Sandusky and J. B. Page. Interrelation between the stability of extended normal modes and the existence of intrinsic localized modes in nonlinear lattices with realistic potentials. Phys. Rev. B, 50:866, 1994.

[157] K. W. Sandusky, J. B. Page, and K. E. Schmidt. Stability and motion of intrinsic localized modes in nonlinear periodic lattices. Phys. Rev., B46:6161, 1992.

[158] S. A. Schofield, R. E. Wyatt, and P. G. Wolynes. Computational study of many-dimensional quantum vibrational energy redistribution. i. statistics of the survival probability. J. Chem. Phys., 105:940, 1996.

[159] A. C. Scott, J. C. Eilbeck, and H. Gilhøj. Quantum lattice solitons. Physica D, 78:194, 1994.

[160] H. Segur and M. D. Kruskal. Nonexistence of small-amplitude breather solutions in $\phi^{4}$ theory. Phys. Rev. Lett., 58:747, 1987.

[161] Z. Shi and G. Huang. Envelope-kink phonon localized modes in a homogeneous anharmonic chain. Phys. Rev., B44:12601, 1991.

[162] A. J. Sievers and J. B. Page. Unusual anharmonic local mode systems. In G. K. Horton and A. A. Maradudin, editors, Dynamical Properties of Solids VII Phonon Physics The Cutting Edge, page 137. Elsevier Amsterdam, 1995.

[163] A. J. Sievers and S. Takeno. Intrinsic localized modes in anharmonic crystals. Phys. Rev. Lett., 61:970, 1988.

[164] A. W. Snyder, D. J. Mitchell, and Yu. S. Kivshar. Unification of linear and nonlinear wave optics. Mod. Phys. Lett. B, 9:1479, 1995.

[165] P. Stancioff, C. R. Willis, M. El-Batanouny, and S. Burdick. Sine-gordon kinks on a discrete lattice. ii. static properties. Phys. Rev., B33:1912, 1986.

[166] R. L. Swofford, M. E. Long and A. C. Albrecht. C-H vibrational states of benzene, naphtalene, and anthracene in the visible region by thermal lensing spectroscopy and the local mode model. J. Chem. Phys., 65::179, 1976.

[167] S. Takeno. P-like stationary self-localized modes in pure one-dimensional lattice with quartic lattice anharmonicity. $J$. Phys. Soc. Japan, 59:3861, 1990.

[168] S. Takeno. Quantum theory of vibron solitons - coherent states of a vibron-phonon system and self-localized modes -. J. Phys. Soc. Japan, 59:3127, 1990.

[169] S. Takeno. Theory of stationary anharmonic localized modes in solids. J. Phys. Soc. Japan, 61:2821, 1992.

[170] S. Takeno and S. Homma. Self-localized anharmonic rotational modes of bases in dna. J. Phys. Soc. Japan, 59:1890, 1990.

[171] S. Takeno and S. Homma. Propagation of a soliton and a nonlinear self-localized state in a one-dimensional disordered nonlinear lattice. J. Phys. Soc. Japan, 60:731, 1991.

[172] S. Takeno and S. Homma. Robust nature of dispersionless envelope lattice solitons and their propagation in onedimensional disordered nonlinear lattices. J. Phys. Soc. Japan, 62:835, 1993.

[173] S. Takeno and K. Hori. A propagating self-localized mode in a one-dimensional lattice with quartic anharmonicity. $J$. Phys. Soc. Japan, 59:3037, 1990.

[174] S. Takeno and K. Hori. Self-localized modes in a pure one-dimensional lattice with cubic and quartic lattice anharmonicity. 
J. Phys. Soc. Japan, 60:947, 1991.

[175] S. Takeno and K. Kawasaki. Intrinsic self-localized magnons in one-dimensional antiferromagnets. Phys. Rev. B, 45:5083, 1992.

[176] S. Takeno, K. Kawasaki, and K. Taniguchi. Exact and approximate analytical solutions of stationary vortexlike modes in the d-dimensional anisotropic classical o(2) (xy) spin model. J. Phys. Soc. Japan, 62:2192, 1993.

[177] S. Takeno, K. Kisoda, and A. J. Sievers. Intrinsic localized vibrational modes in anharmonic crystals. Prog. Theor. Phys. Suppl., 94:242, 1988.

[178] S. Takeno and M. Peyrard. Nonlinear modes in coupled rotator models. Physica, D92:140, 1996.

[179] J. M. Tamga, M. Remoissenet, and J. Pouget. Breathing solitary waves in a sine-gordon two-dimensional lattice. Phys. Rev. Lett., 75:357, 1995.

[180] J. L. Ting and M. Peyrard. Effective breather trapping mechanism for dna transcription. Phys. Rev. E, 53:1011, 1996.

[181] M. Toda. Theory of Nonlinear Lattices. Springer Verlag Berlin, 1989.

[182] R. Trautman-Michalska. Formation of an optical breather. J. Opt. Soc. America, 6:36, 1989.

[183] G. P. Tsironis and S. Aubry. Slow relaxation phenomena induced by breathers in nonlinear lattices. Phys. Rev. Lett., 77:5225, 1996.

[184] A. Tsurui. Wave modulations in anharmonic lattices. Prog. Theor. Phys., 48:1196, 1972.

[185] T. Uzer. Theories of intramolecular vibrational energy transfer. Phys. Rep., 199:73, 1991.

[186] B. G. Vekhter and M. A. Ratner. Spatial and temporal decay of localized electrons in solids: One-dimensional model. J. Chem. Phys., 101:9710, 1994.

[187] B. G. Vekhter and M. A. Ratner. Energy and charge trapping by localized vibrations: Electron-vibrational coupling in anharmonic lattices. Phys. Rev., B51:3469, 1995.

[188] R. F. Wallis, A. Franchini, and V. Bortolani. Localized modes in inhomogeneous one-dimensional anharmonic lattices. Phys. Rev., 50:9851, 1994.

[189] R. F. Wallis, D. L. Mills, and A. D. Boardman. Intrinsic localized spin modes in ferromagnetic chains with on-site anisotropy. Phys. Rev., B52:R3828, 1995.

[190] S. Wang. Localized vibrational modes in an anharmonic chain. Phys. Lett. A, 182:105, 1993.

[191] W. Z. Wang, J. Tinka Gammel, A. R. Bishop, and M. I. Salkola. Quantum breathers in a nonlinear lattice. Phys. Rev. Lett., 76:3598, 1996.

[192] S. Watanabe, S. H. Strogatz, H. S. J. van der Zant, and T. P. Orlando. Whirling modes and parametric instabilities in the discrete sine-gordon equation: experimental tests in josephson rings. Phys. Rev. Lett., 74:23, 1995.

[193] C. R. Willis and R. Boesch. Effect of lattice discreteness on the statistical mechanics of a dilute gas of kinks. Phys. Rev., B41:4570, 1990.

[194] C. R. Willis, M. El-Batanouny, R. Boesch, and P. Sodano. Nonlinear internal-mode influence on the statistical mechanics of a dilute gas of kinks: The double-sine-gordon model. Phys. Rev. B, 40:686, 1989.

[195] C. R. Willis, M. El-Batanouny, and P. Stancioff. Sine-gordon kinks on a discrete lattice. i. hamiltonian formalism. Phys. Rev., B33:1904, 1986.

[196] G. B. Witham. Linear and Nonlinear Waves. Wiley New York, 1974.

[197] G. S. Zavt, M. Wagner, and A. Lütze. Anderson localization and solitonic energy transport in one-dimensional oscillatory systems. Phys. Rev. E, 47:4108, 1993. 


\section{FIGURE CAPTIONS}

Fig.1

$\Psi_{b}(x, t)$ from (1.2) versus $x$ for 26 different times equally spaced and covering one breather period and $m=0.5$.

Fig.2

$e_{(5)}$ versus time (dashed line). Total energy of the chain, solid line. Inset: energy distribution $e_{l}$ versus particle number for the same solution as in Fig. 2 measured for $1000<t<1150$.

Fig.3

Fourier transformed FT $\left[u_{l}(t \geq 1000)\right](\omega)$ with initial condition as in Fig.2 for $l=0$. Inset: for $l= \pm 1$.

Fig.4

Poincare intersection between the trajectory and the subspace $\left[\dot{u}_{1}, u_{1}, u_{0}=0, \dot{u}_{0}>0\right]$ for the symmetric reduced three-particle problem and energy $E=0.58$.

Fig.5

$e_{(5)}(t)$ dependence. Upper short dashed line - total energy of all simulations; solid lines (4) - initial conditions of fixed points in islands 1,2 from Fig. 4 and larger torus in island 1 and torus in island 2 from Fig.4; long dashed line - initial condition of torus in island 3 in Fig.4; dashed-dotted line - initial condition of chaotic trajectory in Fig.4.

Fig.6

Energy distribution for the breather solution with initial energy $E=0.3$ after waiting time $t=3000$. The filled circles represent the energy values for each particle; the solid lines are guides to the eye. Inset: Time dependence of the breather energy $e_{(5)}$.

\section{Fig.7}

Numerical solution for the Fourier components of a discrete breather with $v_{2}=1, v_{4}=-1, \phi_{2}=0.1, N=100$ and frequency $\omega=0.8$. The absolute values of the components $A_{k l}$ are shown as functions of the lattice site $l$ in a window of 30 lattice sites around the breather center. The open squares are the actual results. The lines are guides to the eye and connect components with same Fourier number $k$. $k$ increases from top to bottom as $k=1,3,5,7, \ldots, 23,25$. Fourier components with even $k$ are zero because of the displacement reversal symmetry of the potential.

Fig.8

Slopes of the lines in Fig.7 as a function of $k$ (correspond to the exponents of the decay of the corresponding Fourier components, cf. text) are shown as open squares. The solid line connects the points of the theoretical prediction using the eigenvalues of the linearized map (cf. text).

\section{Fig.9}

Same as in Fig.5 but longer time scale. Note the rapid decrease of $e_{(5)}$ for the solid line around $t=6000$. This 
is due to an internal resonance in the quasiperiodic breather, which induces locally chaotic dynamics, changes the local time spectrum from discrete to continuous and increases the radiation strength by two orders of magnitude.

Fig.10

Poincare intersection (as in Fig.4) where $u$ and $v$ are the position and velocity of the nearest neighbour(s) of the central particle for a two-dimensional lattice (for further details see 84]). The filled circles are the actual mapping results. The lines are guides to the eye and connect the circles in the order of their appearance. The spiral-like form of the broken line indicates the evolution of the contraction of the breather-like object to a fixed point which corresponds to an exact time-periodic discrete breather solution.

Fig.11

Breather energy versus maximum amplitude for the discrete nonlinear Schrödinger system in one, two and three lattice dimensions (cf. text). Parameters $C=0.1$ and $\mu=4$ for all cases. System sizes: $d=1-N=100 ; d=2-N=25^{2}$; $d=3-N=31^{3}$.

The estimated points $(A ; E)$ of bifurcation of the band edge plane wave are: $d=1-(0.014 ; 0.024) ; d=2-(0.064 ; 5.53)$; $d=3-(0.097 ; 237)$.

\section{Fig.12}

Discrete energy density distribution versus lattice site $l$ of a phonon scattering experiment after a waiting time of 12000. The incident phonon wave (from left) has energy density $e_{l}=10^{-4}$ and wave number $q=0.2 \pi$. The breather is positioned at $l=1500$. Since the transmitted part is weak, the reflected wave combines with the incoming wave into a standing wave with wavelength $\lambda / 2=5$. The transmitted wave (right part) shows periodic intensity modulations. Since the group velocity is about 0.04 sites per unit of time, the frequency of the modulation can be estimated to be $\approx 0.023$.

Fig.13

The squared absolute value of the transmission coefficient $|t|^{2}$ as a function of wave number $q$ of the infalling phonon wave. The value $q=\pi$ corresponds to the Brillouin zone boundary. The filled circles are the results of numerical experiments (example in Fig.12). Since the transmitted intensity varies in time, the filled circles represent the timeaveraged intensity. The solid line is the result of the scattering calculation of the linearized phase space flow around the discrete breather by Cretegne (see text).

Fig.14

Quantum energy levels for the DNLS system with 101 sites and two bosons (from [64]). 


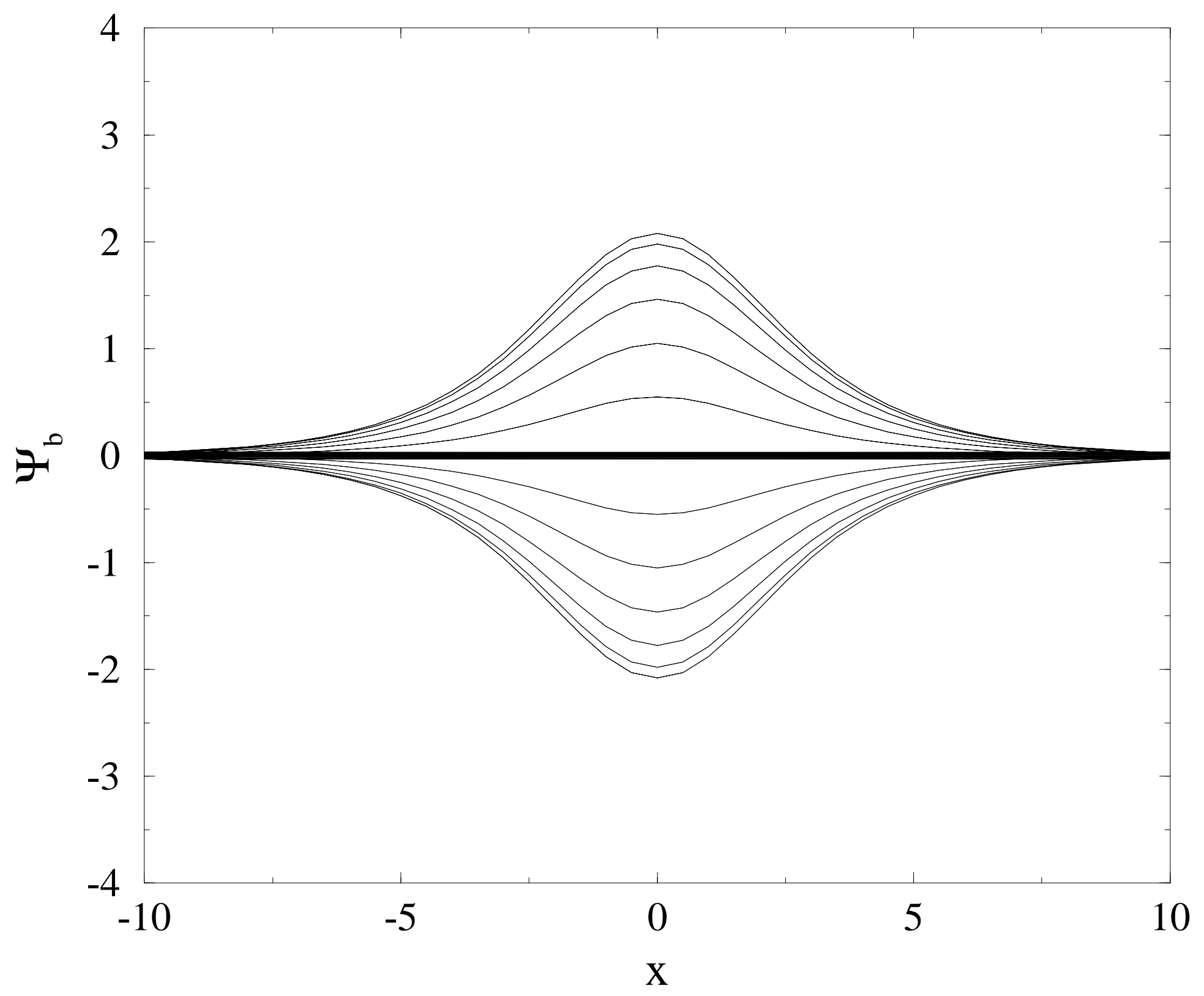

竞 


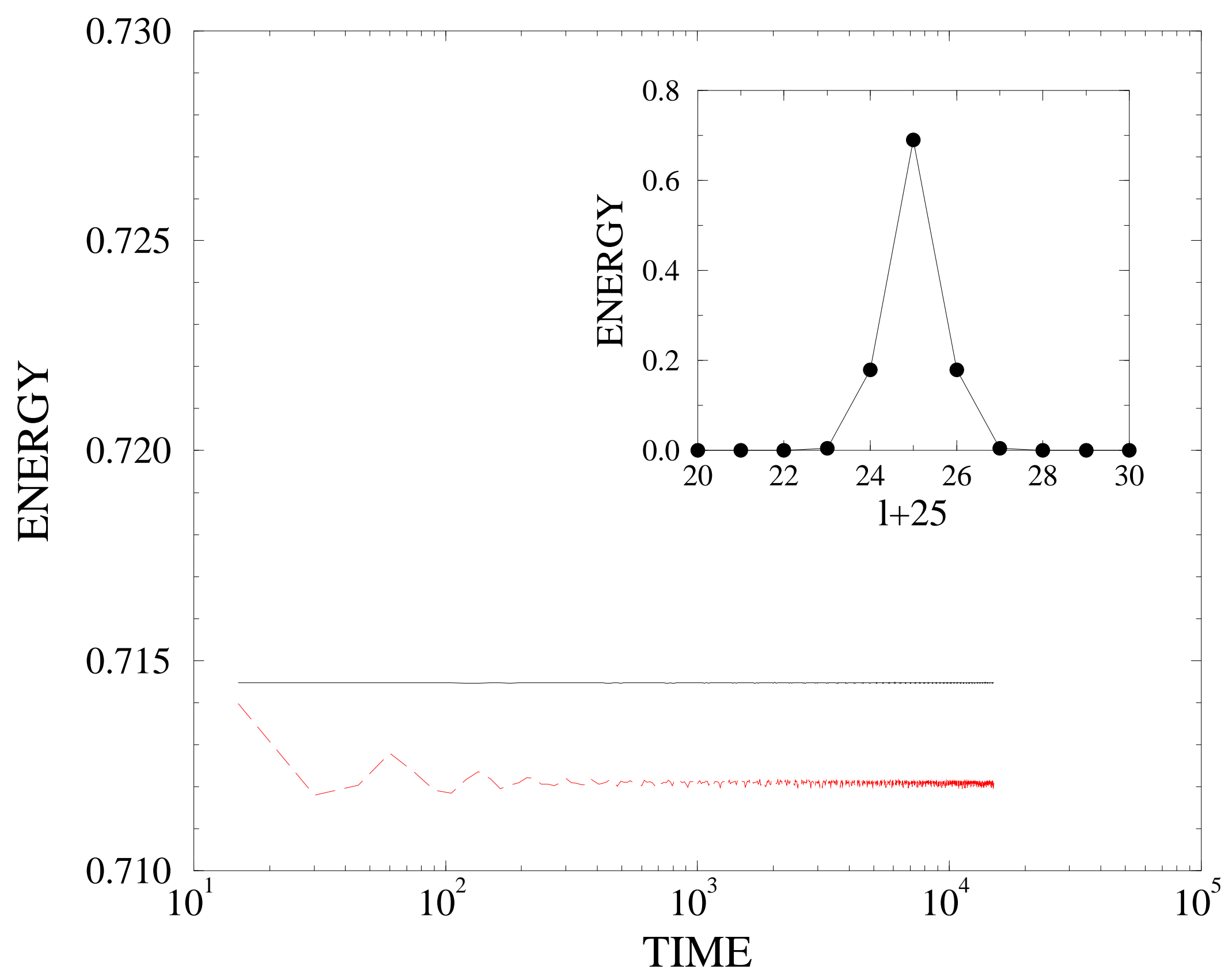

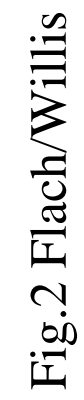




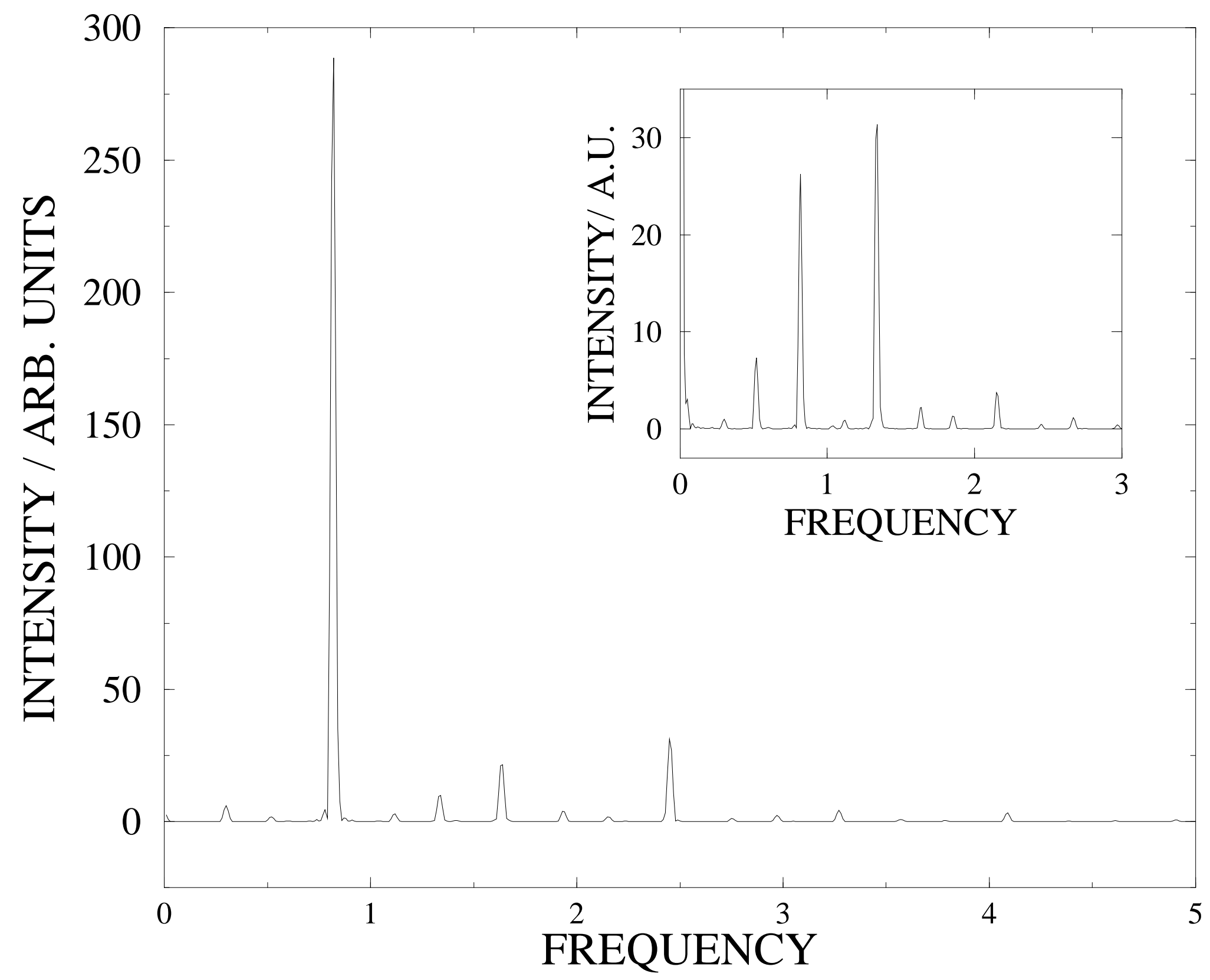

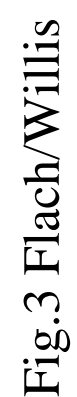




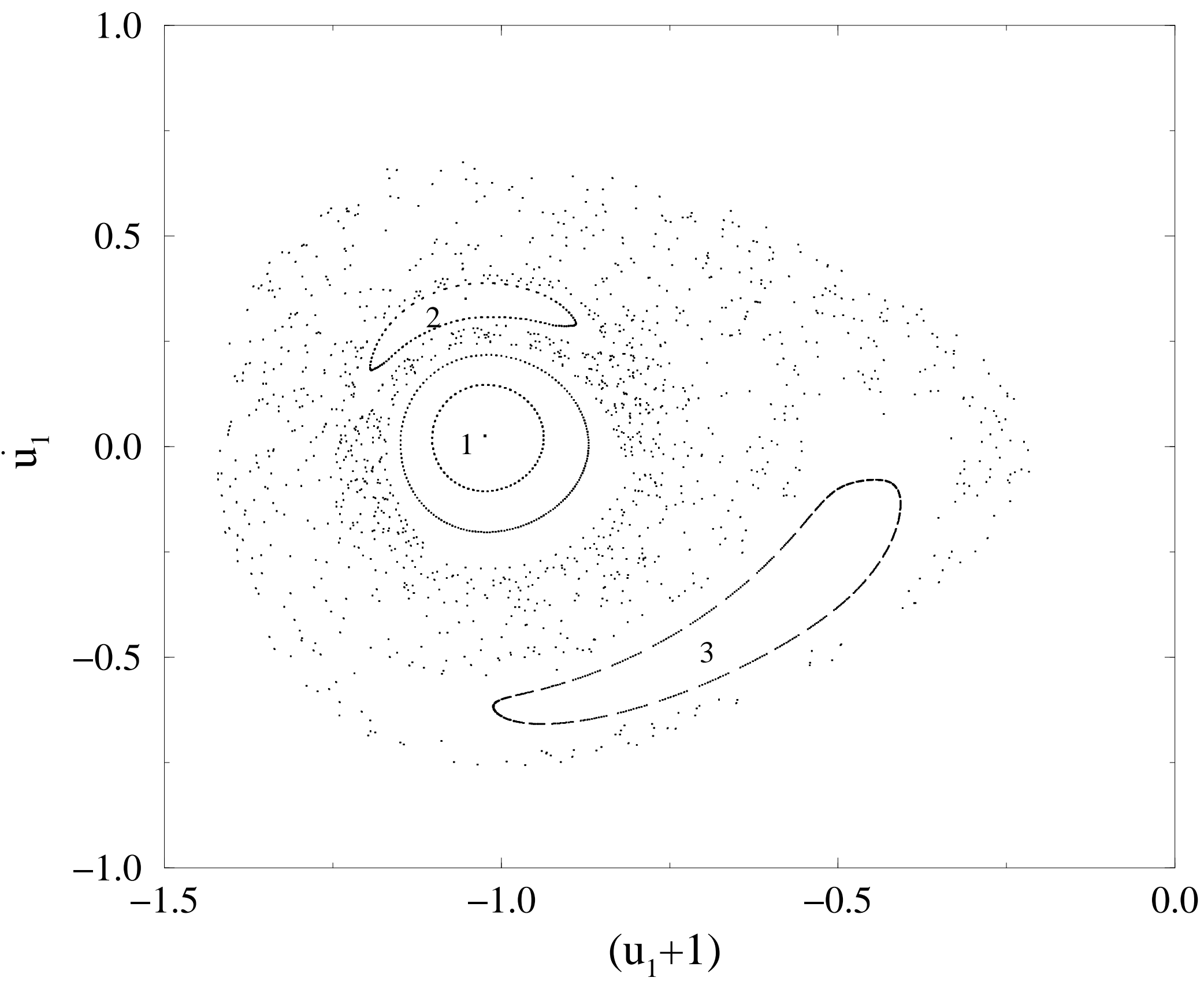

先 


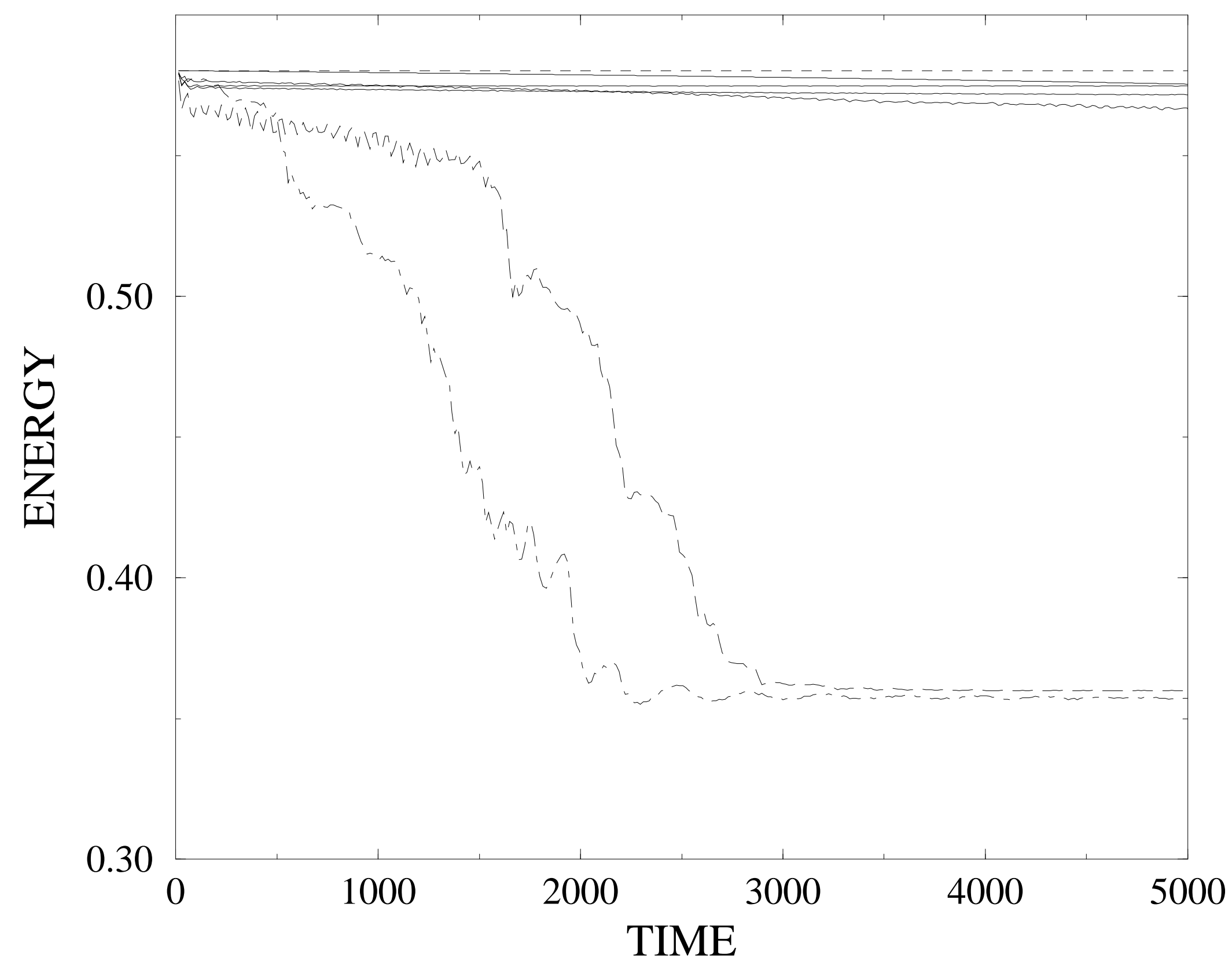

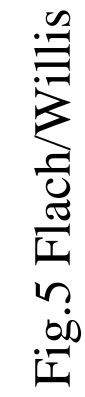




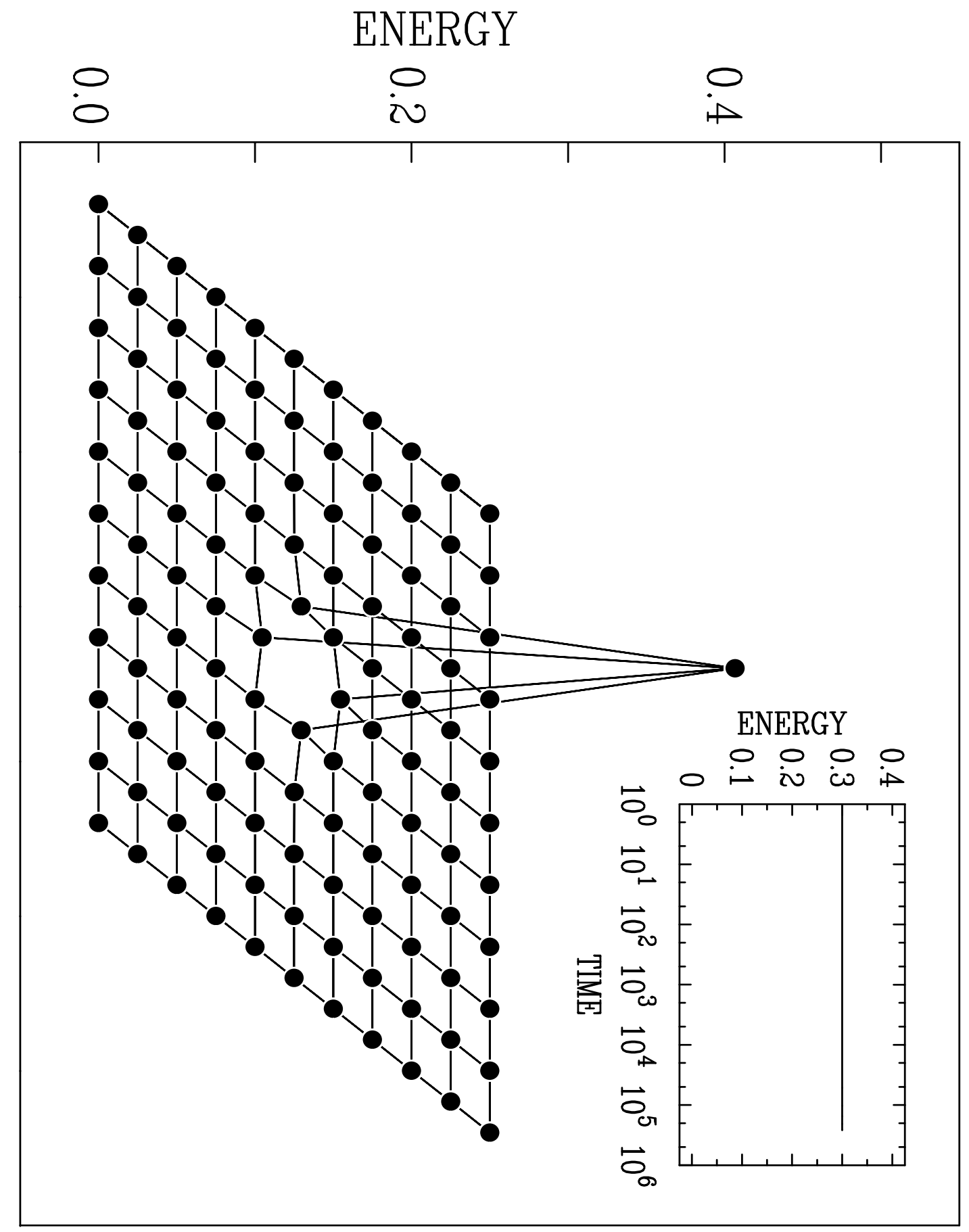




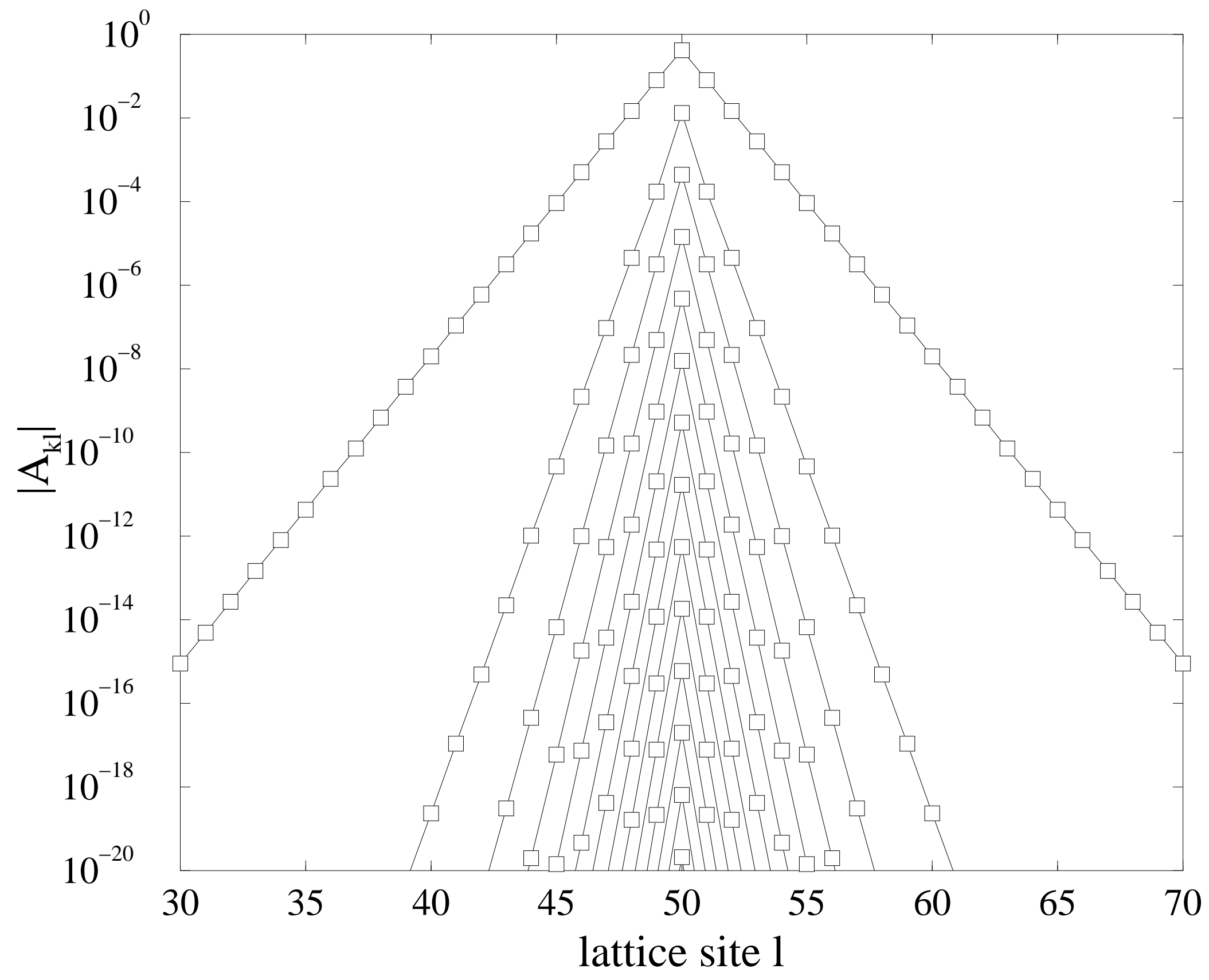

章 


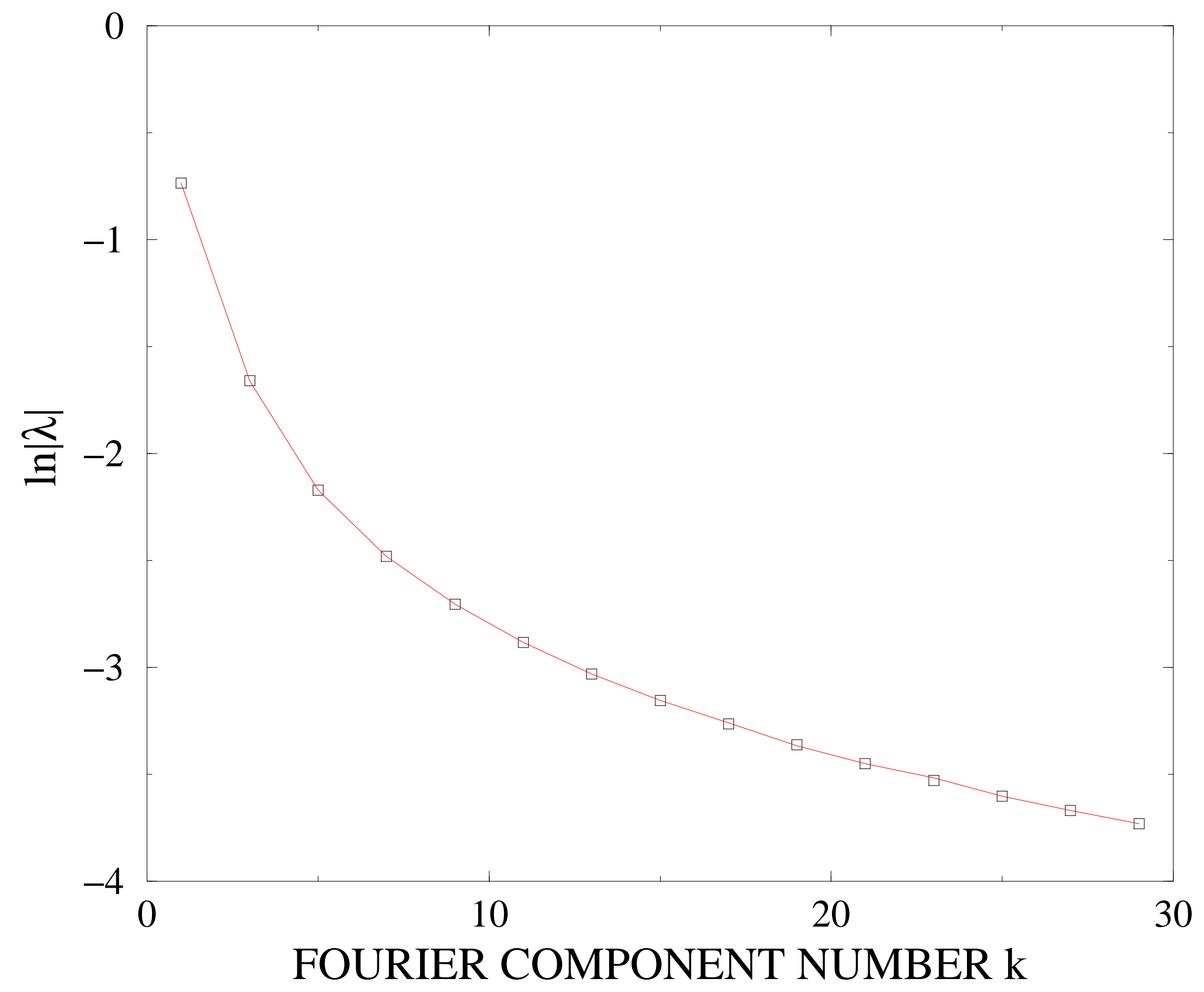

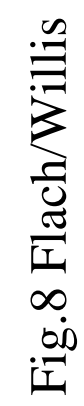




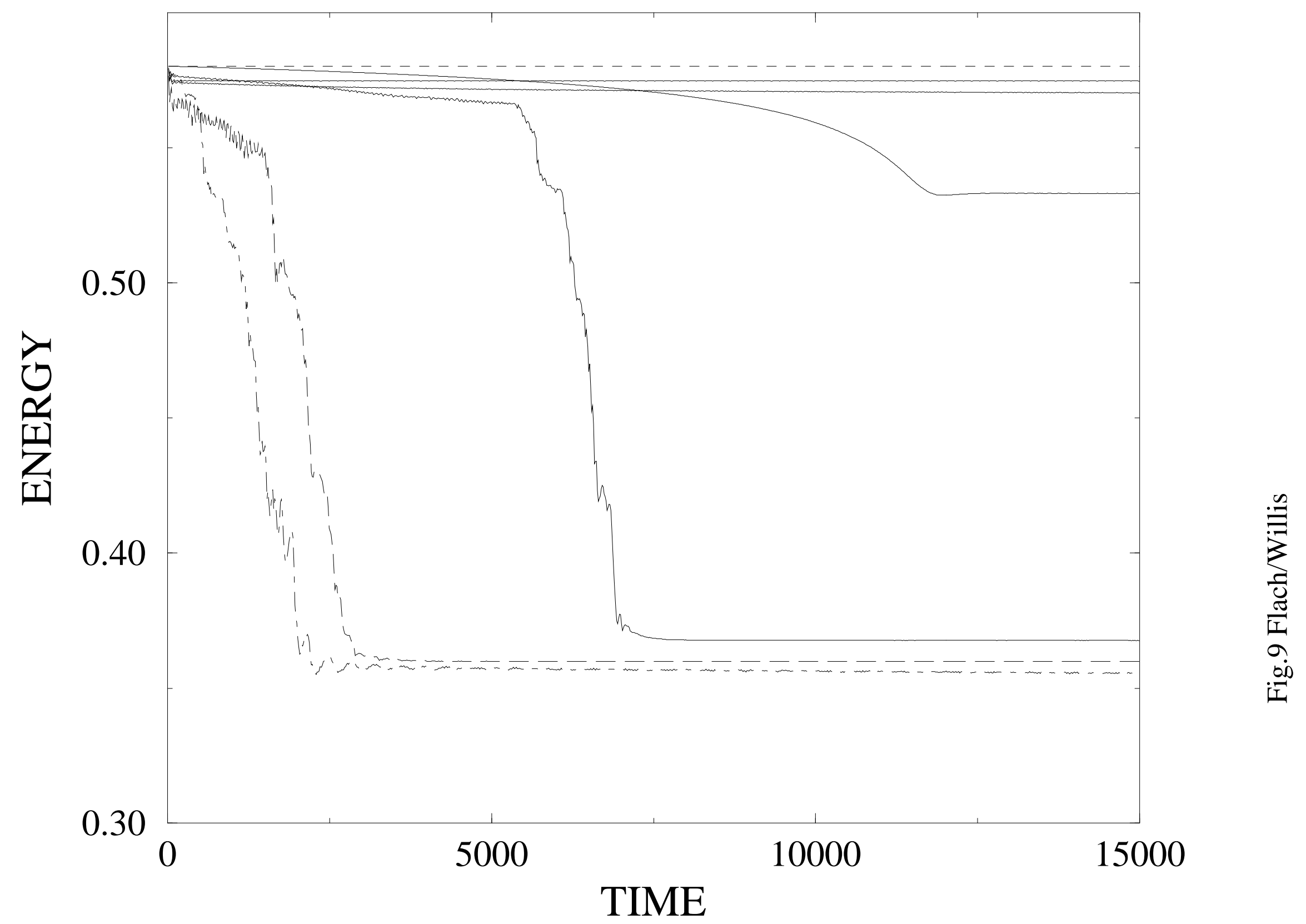




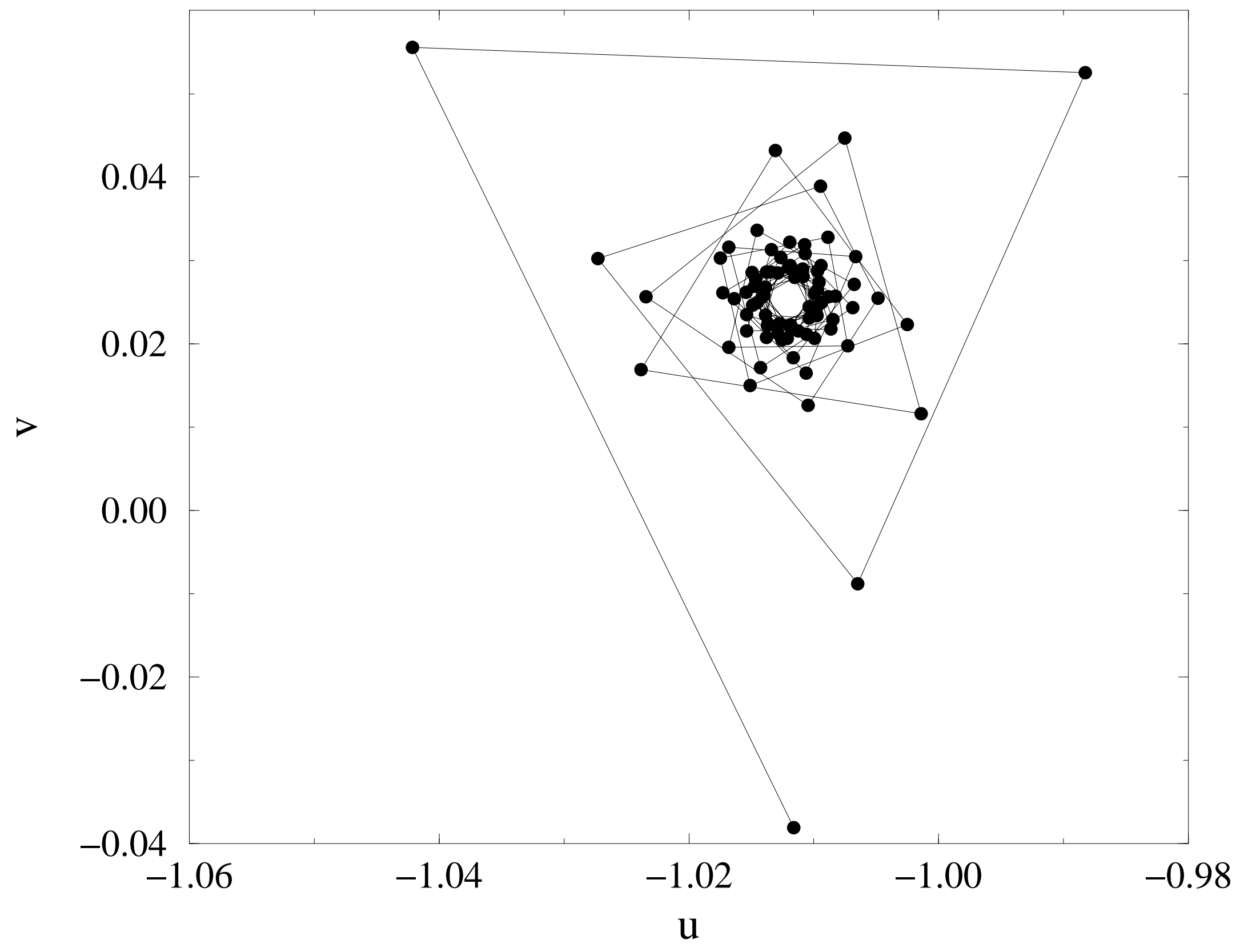

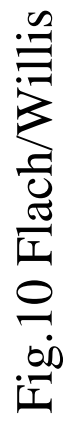




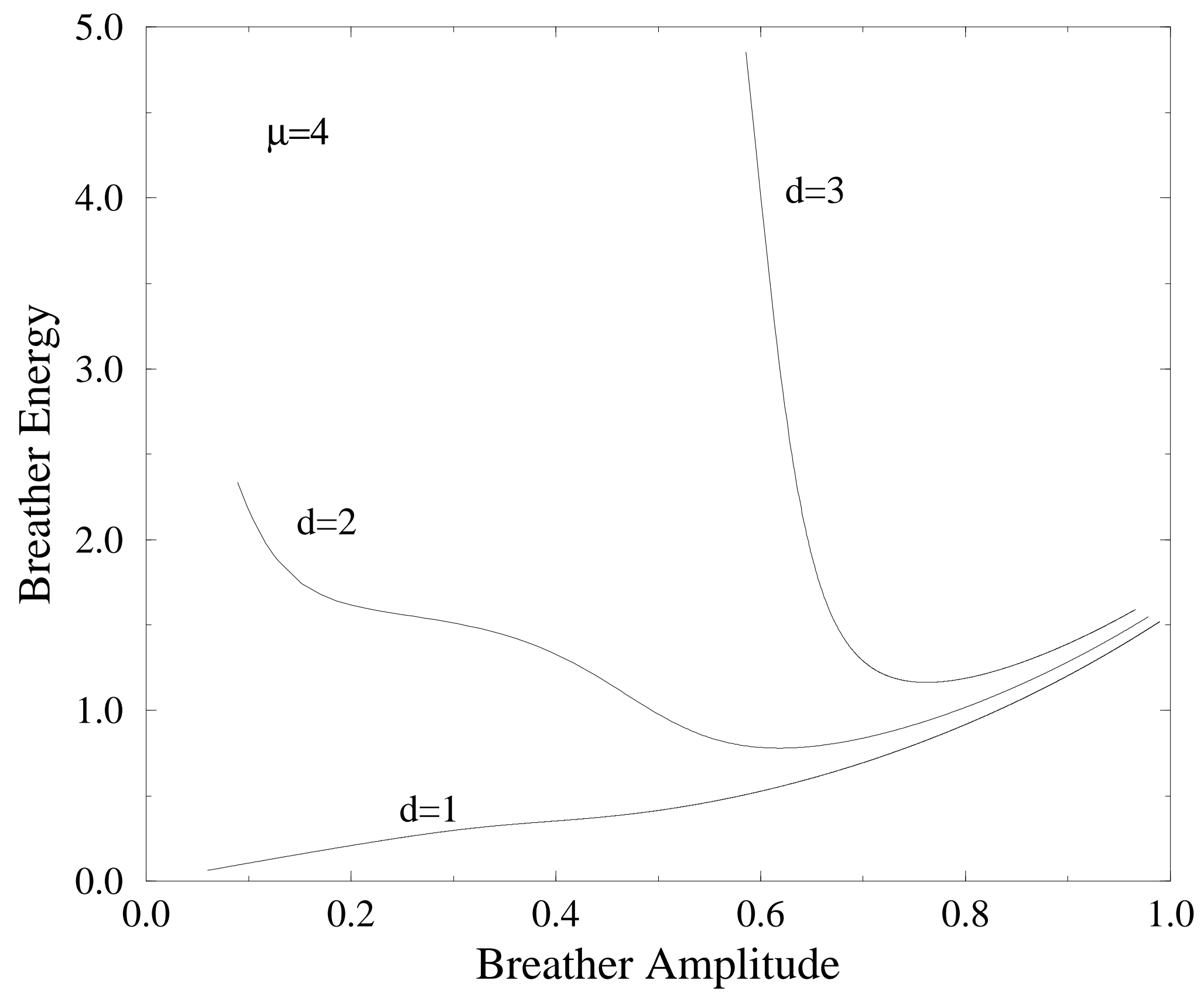

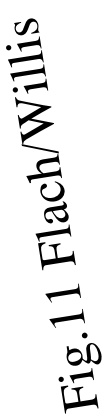




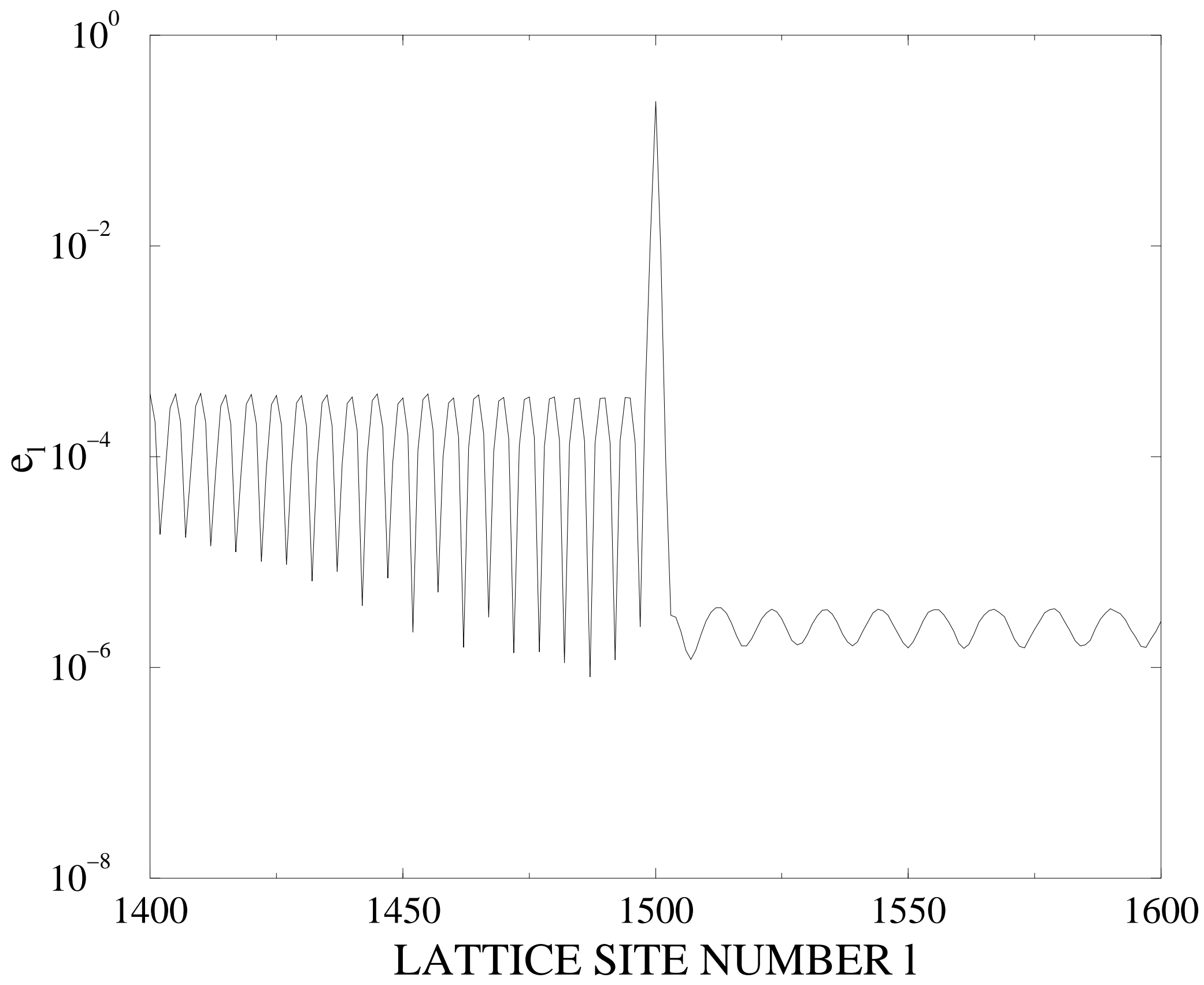

ב্] 


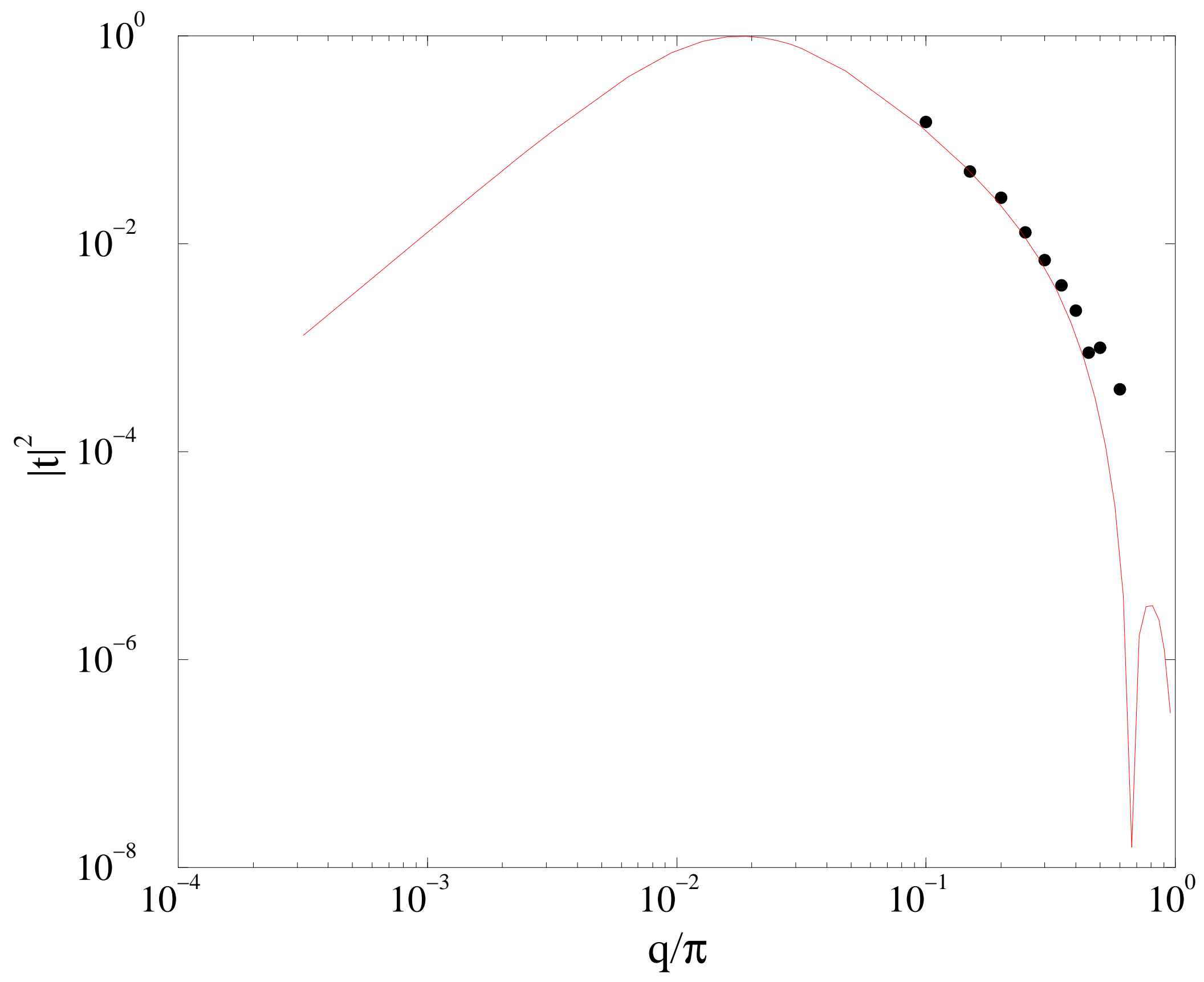

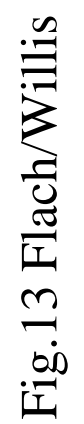




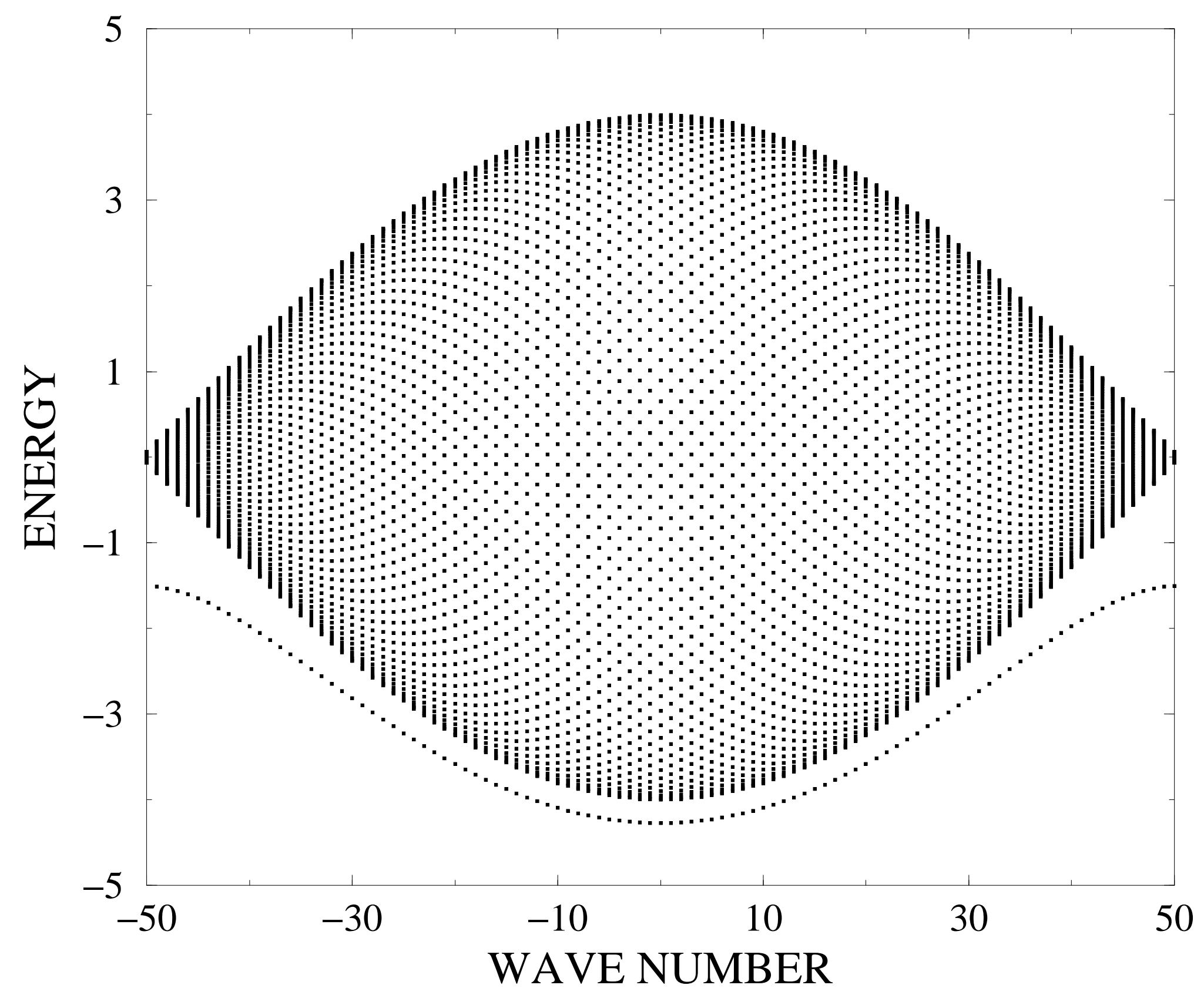

章 